Journal of Mathematical Biology (2017), doi:10.1007/s00285-016-1091-4

\title{
A Bifurcation Theorem for Evolutionary Matrix Models with Multiple Traits
}

\author{
J. M. Cushing ${ }^{\dagger, 1,2}$, F. Martins ${ }^{3}$, A. A. Pinto ${ }^{3}$, Amy Veprauskas ${ }^{2}$ \\ ${ }^{1}$ Department of Mathematics \\ ${ }^{2}$ Interdisciplinary program in Applied Mathematics \\ University of Arizona \\ 617 N Santa Rita \\ Tucson, AZ 85721 \\ ${ }^{3}$ Department of Mathematics \\ Faculty of Sciences \\ University of Porto and LIAAD-INESC \\ Rua do Campo Alegre 687 \\ 4169-007 Porto, Portugal
}

\begin{abstract}
One fundamental question in biology is population extinction and persistence, i.e., stability/instability of the extinction equilibrium and of non-extinction equilibria. In the case of nonlinear matrix models for structured populations, a bifurcation theorem answers this question when the projection matrix is primitive by showing the existence of a continuum of positive equilibria that bifurcates from the extinction equilibrium as the inherent population growth rate passes through 1 . This theorem also characterizes the stability properties of the bifurcating equilibria by relating them to the direction of bifurcation, which is forward (backward) if, near the bifurcation point, the positive equilibria exist for inherent growth rates greater (less) than 1 . In this paper we consider an evolutionary game theoretic version of a general nonlinear matrix model that includes the dynamics of a vector of mean phenotypic traits subject to natural selection. We extend the fundamental bifurcation theorem to this evolutionary model. We apply the results to an evolutionary version of a Ricker model with an added Allee component. This application illustrates the theoretical results and, in addition, several other interesting dynamic phenomena, such as backward bifurcation induced strong Allee effects and survival when multiple traits evolve, but extinction if only one (or no) trait evolves.
\end{abstract}

Key words: Nonlinear matrix models, structured population dynamics, evolutionary game theory, bifurcation, equilibria, stability

AMS subject classifications: 92D25, 92D15, 39A28, 37G99

†Corresponding author: cushing@math.arizona.edu 


\section{Introduction}

We consider a discrete time model

$$
\hat{x}(t+1)=P(\hat{x}(t)) \hat{x}(t), \quad t \in \mathbb{N}_{0}=\{0,1,2, \ldots\}
$$

for the dynamics of a biological population whose individuals are classified into a finite number of discrete classes. Here $\hat{x}: \mathbb{N}_{0} \rightarrow \overline{\mathbb{R}}_{+}^{m}$ is a sequence of $m$-dimensional column vectors consisting of class specific population densities, where $\overline{\mathbb{R}}_{+}^{m}$ is the closure of the positive cone $\mathbb{R}_{+}^{m}$ in $m$-dimensional Euclidean space $\mathbb{R}^{m}$. Recursive formulas (1), called matrix models, are widely utilized to describe the dynamics of populations in which individuals are classified according to age, size, life cycle stage, spatial location, genetic composition, etc., indeed virtually any classification scheme of interest $[4,7]$.

The entries $p_{i j}(\hat{x})$ of the projection matrix $P(\hat{x})$ are chosen by a modeler to describe classspecific, per capita (individual) birth and survival rates and to account for transitions of individuals from one class to another. As indicated, these entries can be dependent on the densities in the demographic vector $\hat{x}$, dependencies that make the dynamic model nonlinear. Classic examples of matrix models for structured population dynamics include the age, size, and stage structured models of Leslie and Lewis [19, 20, 21], Usher [26], and Lefkovitch [18].

Of fundamental importance to a biological population is its avoidance of extinction. We refer to the equilibrium $\hat{x}=\hat{0}$ solution of (1) as the extinction equilibrium. If the extinction equilibrium is an attractor, then the population is threatened with extinction. This leads to the study of the stability properties (local and global) of the extinction equilibrium. The linearization principle [14] leads one to consider the eigenvalues of the Jacobian obtained from (1) evaluated at the extinction equilibrium, which is the inherent projection matrix $P(\hat{0})$ (inherent means density free). If all eigenvalues of $P(\hat{0})$ lie in the complex unit circle, then the extinction equilibrium is locally asymptotically stable ${ }^{1}$, which threatens the model population with (asymptotic) extinction. If at least one eigenvalue is outside the complex unit circle, then the extinction equilibrium is unstable, which opens the possibility of population persistence. The nature of the bifurcation that occurs when the extinction equilibrium loses stability ${ }^{2}$ forms a fundamental bifurcation theorem in population dynamics. We describe this theorem below (Theorem 1).

In the matrix model (1), the vital rates and transitions modeled by the entries $p_{i j}(\hat{x})$ of the projection matrix $P(\hat{x})$ change temporally only due to changes in the demographic vector $\hat{x}=$ $\hat{x}(t)$. There are, of course, numerous other reasons why these vital rates and transitions might change in time, for example, they might fluctuate randomly due to demographic or environmental stochasticity or periodically due to regular environmental oscillations (seasonal, monthly or daily fluctuations). Another reason these vital rates and transitions might change in time is that they are subject to selective pressures from Darwinian evolution. Our goal in this paper is to investigate an extension of the fundamental bifurcation theorem for the non-evolutionary model (1), as given in Theorem 1 below, to an evolutionary game theoretic version of (1). We describe the evolutionary model in Section 3, study the stability of an extinction equilibrium in Section 4, and in Section 5 determine the nature of the bifurcation that occurs when extinction stability is lost. In Section 6

\footnotetext{
${ }^{1}$ An equilibrium $\hat{x}$ is locally stable if given any $\varepsilon>0$ there exists a $\delta>0$ such that for any initial condition satisfying $|\hat{x}(0)-\hat{x}|<\delta$ it follows that the solution satisfies $|\hat{x}(t)-\hat{x}|<\varepsilon$ for all $t \in N_{0}$. An equilibrium is a local attractor if there exists a $\delta_{0}>0$ such that $|\hat{x}(0)-\hat{x}|<\delta$ implies $\lim _{t \rightarrow+\infty} \hat{x}(t)=\hat{x}$. An equilibrium is locally asymptotically stable if it is both locally stable and a local attractor.

${ }^{2}$ Throughout this paper stable (or stability) means local asymptotically stable (or local asymptotic stability). Unstable means not locally asymptotically stable.
} 
an application is made to an evolutionary version of a Ricker model with an added Allee component (low density positive feedback effect).

\section{A Bifurcation Theorem for the Matrix Model (1).}

We make the following assumptions on the entries $p_{i j}(\hat{x})$ in the projection matrix $P(\hat{x})$. Let $\Omega \subseteq \mathbb{R}^{m}$ denote an open neighborhood of $\hat{0} \in \mathbb{R}^{m}$ and $C^{2}\left(\Omega \rightarrow \overline{\mathbb{R}}_{+}\right)$denote the set of twice continuously differentiable functions that map $\Omega$ to $\overline{\mathbb{R}}_{+}$.

H1: $P(\hat{x})=\left[p_{i j}(\hat{x})\right]$ is primitive for all $\hat{x} \in \Omega$ and $p_{i j} \in C^{2}\left(\Omega \rightarrow \overline{\mathbb{R}}_{+}\right)$.

Recall that a nonnegative matrix (i.e. one all of whose entries are nonnegative) is primitive if it is irreducible and has a strictly dominant eigenvalue. Perron-Frobenius theory implies that the spectral radius $\rho[A]$ of a primitive matrix $A$ is a strictly dominant, positive and simple eigenvalue which possesses a positive eigenvector in $\mathbb{R}_{+}^{m}$. Moreover, no other eigenvalue has a nonnegative eigenvector, i.e. an eigenvector in $\overline{\mathbb{R}}_{+}^{m}$. See [3]. We denote the strictly dominant eigenvalue of $P(\hat{x})$ by

$$
r(\hat{x}):=\rho[P(\hat{x})] .
$$

Observe that $r(\cdot) \in C^{2}\left(\Omega \rightarrow \mathbb{R}_{+}^{1}\right)$. The number $r(\hat{0})$ is the inherent growth rate of the population (the growth rate in the absence of density effects). For notational simplicity we denote this number by

$$
r_{0}:=r(\hat{0}) .
$$

For our purposes, we normalize the entries of $P$ in a way that

$$
P(\hat{x})=r_{0} Q(\hat{x})
$$

where the normalized matrix $Q(\hat{x})=\left[q_{i j}(\hat{x})\right]$ satisfies H1 and

$$
\rho[Q(\hat{0})]=1 .
$$

Then the matrix equation (1) becomes

$$
\hat{x}(t+1)=r_{0} Q(\hat{x}(t)) \hat{x}(t), \quad t \in \mathbb{N}_{0} .
$$

We denote the entries of the matrix $Q(\hat{x})$ by $q_{i j}(\hat{x})$.

Definition 1 We say that a pair $\left(r_{0}, \hat{x}\right) \in \mathbb{R} \times \Omega$ is an equilibrium pair of (2) (or equivalently of (1)) if $\hat{x}=r_{0} Q(\hat{x}) \hat{x}$. Observe that $\left(r_{0}, \hat{0}\right)$ is an equilibrium pair for every $r_{0} \in \mathbb{R}$; we call $\left(r_{0}, \hat{0}\right)$ an extinction equilibrium pair. An equilibrium pair $\left(r_{0}, \hat{x}\right)$ is a positive equilibrium pair if $\hat{x} \in \mathbb{R}_{+}^{m}$ and it is stable if $\hat{x}$ is a locally asymptotically stable equilibrium of (2) (equivalently (1)).

We need the quantity

$$
\kappa:=-\hat{w}_{L}^{T}\left[\nabla_{\hat{x}}^{0} q_{i j}^{T} \hat{w}_{R}\right] \hat{w}_{R}
$$

where $T$ denotes transposition, the gradient $\nabla_{\hat{x}}$ of $q_{i j}(\hat{x})$ with respect to $\hat{x}$ is a column $m$-vector, and $\nabla_{\hat{x}}^{0} q_{i j}^{T}$ denotes the transpose of the gradient evaluated at the bifurcation point $\left(r_{0}, \hat{x}\right)=(1, \hat{0})$. With this superscript notational convention, we can equivalently write

$$
\kappa=-\hat{w}_{L}^{T}\left[\nabla_{\hat{x}}^{0} p_{i j}^{T} \hat{w}_{R}\right] \hat{w}_{R} .
$$


Here the vectors $\hat{w}_{L}^{T}$ and $\hat{w}_{R}$ are the (positive) left and right eigenvectors of $Q(\hat{0})$ (equivalently of $P(\hat{0})$ when $\left.r_{0}=1\right)$ associated with eigenvalue 1 , normalized so that

$$
\hat{w}_{L}^{T} \hat{w}_{R}=1 .
$$

Note that $\left[\nabla_{\hat{x}}^{0} p_{i j}^{T} \hat{w}_{R}\right]$ is an $m \times m$ matrix. The derivative $\partial_{x_{k}}^{0} p_{i j}$ measures the effect that an increase in the density of class $k$ has on the entry $p_{i j}$ of the population projection matrix $P$ (at low population density). The number $\hat{w}_{L}^{T}\left[\nabla_{\hat{x}}^{0} p_{i j}^{T} \hat{w}_{R}\right] \hat{w}_{R}$ is a weighted sum (with positive coefficients) of all density effects on all entries $p_{i j}$. This number therefore represents a summary measure of the effects that (low level) class densities has on the population (as does $\kappa$, the minus sign being introduced only for notational convenience in Theorem 1.)

From the linearization principle and from Theorems 1.2.4 and 1.2.5 in [7] we have the following result.

Theorem 1 Assume the matrix $P(\hat{x})$ in (1) satisfies $H 1$.

(a) The extinction equilibrium $\left(r_{0}, \hat{0}\right)$ is stable for $r_{0}<1$ and is unstable for $r_{0}>1$.

(b) There exists a continuum $\mathcal{C}$ of positive equilibrium pairs $\left(r_{0}, \hat{x}\right) \in \mathbb{R}_{+} \times \mathbb{R}_{+}^{m}$ of the matrix equation (1) which bifurcates from $(1, \hat{0})$ (i.e. contains the extinction pair $(1, \hat{0})$ in its closure). Near the bifurcation point, the positive equilibrium pairs on $\mathcal{C}$ have the parameterization

$$
\begin{aligned}
\hat{x}(\varepsilon) & =\hat{w}_{R} \varepsilon+\mathcal{O}\left(\varepsilon^{2}\right) \\
r_{0}^{*}(\varepsilon) & =1+\kappa \varepsilon+\mathcal{O}\left(\varepsilon^{2}\right)
\end{aligned}
$$

for $\varepsilon \gtrsim 0$.

(c) We say the bifurcation of positive equilibria is forward (respectively, backward) if, in a neighborhood of $(1, \hat{0})$, the positive equilibrium pairs on $\mathcal{C}$ are such that $r_{0}>1$ (respectively, $\left.r_{0}<1\right)$. If $\kappa>0$ then the bifurcation of $\mathcal{C}$ at $(1, \hat{0})$ is forward and the equilibrium pairs on $\mathcal{C}$ in a neighborhood of $(1, \hat{0})$ are (locally asymptotically) stable. If $\kappa<0$ then the bifurcation is backward and the equilibrium pairs on $\mathcal{C}$ in a neighborhood of $(1, \hat{0})$ are unstable.

Note how, in this Theorem, the direction of the bifurcation determines the stability of the bifurcating equilibria. A forward bifurcation, occurring when the extinction equilibrium loses its stability as $r_{0}$ increases through 1 (removing the threat of extinction), creates stable positive (nonextinction) equilibrium states.

Theorem 1 asserts stability or instability of the bifurcating positive equilibria $\mathcal{C}$ locally only, i.e. for equilibrium pairs on $\mathcal{C}$ near the extinction equilibrium $(1, \hat{0})$ only. However, the continuum $\mathcal{C}$ is known to exist globally in the sense that it connects to the boundary of the set on which the matrix model is defined, i.e., it connects to the set $\{+\infty\} \times\left(\partial \Omega \cap \mathbb{R}_{+}^{m}\right)$, where $\partial \Omega$ denotes the boundary of $\Omega$. In most applications, $\Omega$ includes the closure $\overline{\mathbb{R}}_{+}^{m}$ of the positive cone, which implies that either the component $r_{0}$ is unbounded or the norm $|\hat{x}|$ is unbounded in $\mathbb{R}_{+}$(or both). When $r_{0}$ is unbounded we have that there is at least one non-extinction equilibrium for each $r_{0}>1[7,8]$.

A derivative $\partial_{x_{k}} p_{i j}$ is often negative in population models because of an assumption that an increase in density $x_{k}$ will have a deleterious effect on some vital rate (birth rate, survival probability, growth rate, metabolic rates, and so on). These kinds of negative feedback phenomena are common in population models that describe density regulation mechanisms for population growth. If all the derivatives $\partial_{x_{k}}^{0} p_{i j}$ are negative (or zero), that is to say, if all density effects in a model are negative feedback effects, then clearly $\kappa>0$ and the bifurcation of the continuum $\mathcal{C}$ is forward and hence stable. 
A positive derivative $\partial_{x_{k}}^{0} p_{i j}$ is called a component Allee effect [5]. Clearly, the existence of a component Allee effect is necessary for a backward bifurcation (i.e. for $\kappa<0$ ). If all component Allee effects are sufficiently large so that $\kappa<0$, then the bifurcation of positive equilibria at $(1, \hat{0})$ is backward and hence unstable. A common occurrence in this case is the creation of a strong Allee effect, i.e. the presence of two attractors, one of which is an extinction equilibrium and the other of which is positive. Thus, population survival is initial condition dependent. This scenario can only occur when $r_{0}<1$ and the extinction equilibrium is stable. A backward bifurcation does not create a stable positive equilibrium, however. A strong Allee effect usually arises in models with backward bifurcations. This is because it is usually assumed that negative feedback effects predominate at high densities (even if they do not at low densities) which has the consequence of "turning" the continuum $\mathcal{C}$ around at a critical (saddle-node bifurcation) value of $r_{0}<1$ with a concomitant stabilization of the positive equilibria. We will not pursue this phenomena here, which occurs outside a neighborhood of the bifurcation point. See [10].

In this paper we extend the fundamental bifurcation Theorem 1 to an evolutionary version of the matrix model (2) under the assumption that the projection matrix depends on a suite of phenotypic traits subject to natural selection. This generalizes the results in [6] where models with only a single trait are considered.

\section{Darwinian Dynamics with Multiple Evolutionary Traits}

We consider an evolutionary version of the matrix model (1) developed in [28]. In that modeling methodology a (focal) individual's vital rates, as described by the entries of the projection matrix, are influenced by a collection of scalar traits $\hat{v}=\left(v_{1}, \ldots, v_{n}\right)^{T}$ and the population means of these traits $\hat{u}=\left(u_{1}, \ldots, u_{n}\right)^{T}$. By this assumption, an individual's fitness depends on both its own suite of traits $\hat{v}$ and the traits possessed by other individuals in the population $\hat{u}$ (frequency dependence). We indicate this by the notation $P(\hat{x}, \hat{u}, \hat{v})$, which in turn implies that the spectral radius of $P(\hat{x}, \hat{u}, \hat{v})$ is also dependent on $\hat{v}$ and $\hat{u}$ :

$$
r(\hat{x}, \hat{u}, \hat{v}):=\rho[P(\hat{x}, \hat{u}, \hat{v})] .
$$

Darwinian dynamics track the dynamics of the structured population $\hat{x}(t)$ and the vector of population mean traits $\hat{u}(t)=\left(u_{1}(t), \ldots, u_{n}(t)\right)^{T}$, the latter by means of the assumption that changes in the mean trait are proportional to the fitness gradient of the focal individual $[1,13,2,16,17,22,28]$. We extend the resulting evolutionary matrix model, as found in [28], to include a vector of traits $\hat{v}=\left(v_{1}, \ldots, v_{n}\right)^{T}$. Different fitness functions can be found throughout the literature, but the most common choice is the exponential growth rate $\ln r$ [24]. Another choice used by some researchers is the net reproduction number $R_{0}(\hat{x}, \hat{u}, \hat{v})$. We use $\ln r(\hat{x}, \hat{u}, \hat{v})$, but note that by the results in [9] our results remain unchanged if $r(\hat{x}, \hat{u}, \hat{v})$ is replaced by $R_{0}(\hat{x}, \hat{u}, \hat{v})$.

The model equations for the coupled population and trait dynamics provided by evolutionary game theory are $[22,28]$

$$
\begin{aligned}
& \hat{x}(t+1)=\left.P(\hat{x}, \hat{u}, \hat{v})\right|_{(\hat{x}, \hat{u}, \hat{v})=(\hat{x}(t), \hat{u}(t), \hat{u}(t))} \hat{x}(t) \\
& \hat{u}(t+1)=\hat{u}(t)+\left.M \nabla_{\hat{v}} \ln r(\hat{x}, \hat{u}, \hat{v})\right|_{(\hat{x}, \hat{u}, \hat{v})=(\hat{x}(t), \hat{u}(t), \hat{u}(t))}
\end{aligned}
$$

where $M=\left(\sigma_{i j}\right)$ is a symmetric $n \times n$ variance-covariance matrix for trait evolution and the gradient 
$\nabla_{\hat{v}} \ln r(\hat{x}, \hat{u}, \hat{v})$ is a column n-vector, whose $i^{\text {th }}$ entry is

$$
\partial_{v_{i}} \ln r(\hat{x}, \hat{u}, \hat{v}):=\frac{\partial \ln r(\hat{x}, \hat{u}, \hat{v})}{\partial v_{i}} .
$$

The entry $\sigma_{i j}$ of $M, i \neq j$, is the covariance of the $i^{t h}$ phenotypic trait and the $j^{t h}$ phenotypic trait. The diagonal entries

$$
\sigma_{i}^{2}:=\sigma_{i i} \geq 0
$$

are the variances of the $i^{t h}$ trait (from its mean $u_{i}$ ) occurring in the population at each time $t$ (which are assumed constant). We assume the usual conditions for a covariance matrix, namely that $M$ is positive semi-definite and symmetric. ${ }^{3}$ If the matrix $M$ is the null matrix, then no evolution occurs and $\hat{u}(t)$ remains constant for all $t$. In this case Theorem 1 holds when applied to (4) with the mean trait $\hat{u}(t) \equiv \hat{u}(0)$ held fixed.

We write (4) and (5) as

$$
\begin{aligned}
& \hat{x}(t+1)=P(\hat{x}(t), \hat{u}(t), \hat{u}(t)) \hat{x}(t) \\
& \hat{u}(t+1)=\hat{u}(t)+M \nabla_{\hat{v}} \ln r(\hat{x}(t), \hat{u}(t), \hat{u}(t))
\end{aligned}
$$

where we use the simplifying notation

$$
\begin{aligned}
P(\hat{x}(t), \hat{u}(t), \hat{u}(t)) \quad: \quad=\left.P(\hat{x}, \hat{u}, \hat{v})\right|_{(\hat{x}, \hat{u}, \hat{v})=(\hat{x}(t), \hat{u}(t), \hat{u}(t))} \\
\nabla_{\hat{v}} \ln r(\hat{x}(t), \hat{u}(t), \hat{u}(t)) \quad: \quad=\left.\left[\nabla_{\hat{v}} \ln r(\hat{x}, \hat{u}, \hat{v})\right]\right|_{(\hat{x}, \hat{u}, \hat{v})=(\hat{x}(t), \hat{u}(t), \hat{u}(t))} .
\end{aligned}
$$

Remark 1 We will need to differentiate functions of the three variables $(\hat{x}, \hat{u}, \hat{v})$ after letting $\hat{v}=\hat{u}$ with respect to the components $u_{i}$ of $\hat{u}$ and from them construct gradients and Jacobians with respect to $\hat{u}$. Such a derivative is the sum of the partial derivatives with respect to $u_{i}$ and $v_{i}$. For example, the derivative of $r(\hat{x}, \hat{u}, \hat{u}):=\left.r(\hat{x}, \hat{u}, \hat{v})\right|_{\hat{v}=\hat{u}}$ with respect to $u_{i}$ is

$$
\frac{\partial}{\partial u_{i}}\left[\left.r(\hat{x}, \hat{u}, \hat{v})\right|_{\hat{v}=\hat{u}}\right]+\frac{d}{d v_{i}}\left[\left.r(\hat{x}, \hat{u}, \hat{v})\right|_{\hat{v}=\hat{u}}\right]
$$

which we write as

$$
\frac{\partial r(\hat{x}, \hat{u}, \hat{u})}{\partial u_{i}}+\frac{\partial r(\hat{x}, \hat{u}, \hat{u})}{\partial v_{i}} .
$$

With this notation, the gradient of $r(\hat{x}, \hat{u}, \hat{u})$ with respect to the components $u_{i}$ of $\hat{u}$ constructed from these partial derivatives is

$$
\nabla_{\hat{u}} r(\hat{x}, \hat{u}, \hat{u})+\nabla_{\hat{v}} r(\hat{x}, \hat{u}, \hat{u}) .
$$

Let $V$ be an open connected set in $\mathbb{R}^{n}$ and let $\Omega \subseteq \mathbb{R}^{m}$ be an open set containing the origin $\hat{0} \in \mathbb{R}^{m}$. We assume the following about the projection matrix $P(\hat{x}, \hat{u}, \hat{v})$ and the variancecovariance matrix $V$.

H2. $P(\hat{x}, \hat{u}, \hat{v})$ is primitive for $(\hat{x}, \hat{u}, \hat{v}) \in \Omega \times V \times V, p_{i j} \in C^{2}\left(\Omega \times V \times V \rightarrow \overline{\mathbb{R}}_{+}\right)$, $p_{i j}(\hat{x}, \hat{u}, \hat{v})=\tilde{p}_{i j}(\hat{v}) \bar{p}_{i j}(\hat{x}, \hat{u}, \hat{v})$ such that $\bar{p}_{i j}(\hat{0}, \hat{u}, \hat{v}) \equiv 1$, and $M$ is invertible.

\footnotetext{
${ }^{3} M$ is positive semi-definite means $\hat{v}^{T} M \hat{v} \geq 0$ for all $\hat{v} \in R^{m} . M$ is symmetric means $\sigma_{i j}=\sigma_{j i}$ for all $1 \leq i, j \leq m$.
} 
Remark 2 The assumption on $p_{i j}$ in $\mathbf{H} 2$ implies that trait frequency dependence has no effect in the absence of density effects. Specifically, $p_{i j}(\hat{0}, \hat{u}, \hat{v})=\tilde{p}_{i j}(\hat{v})$. A mathematical implication of this assumption is that all derivatives of $p_{i j}(\hat{0}, \hat{u}, \hat{v})$ with respect to components $u_{i}$ of $\hat{u}$ are identically equal to 0 for all $\hat{v}$ :

$$
\nabla_{\hat{u}} p_{i j}(\hat{0}, \hat{u}, \hat{v}) \equiv 0_{n} .
$$

This means the inherent projection matrix $P(\hat{0}, \hat{u}, \hat{v})$ is independent of $\hat{u}$ and hence so is its dominant eigenvalue $r(\hat{0}, \hat{u}, \hat{v})$. Thus $\nabla_{\hat{u}} r(\hat{0}, \hat{u}, \hat{v}) \equiv 0_{n}$ for all $\hat{v}$ hence $\nabla_{\hat{u}}\left[\left.r(\hat{0}, \hat{u}, \hat{v})\right|_{\hat{v}=\hat{u}}\right] \equiv$ $0_{n}$. Using the notation convention in Remark 1 we have

$$
\nabla_{\hat{u}} r(\hat{0}, \hat{u}, \hat{u}) \equiv 0_{n} .
$$

Remark 3 The assumption on $M$ in H2, that it is invertible, is for example satisfied if traits are not strongly correlated.

Our approach is to consider the bifurcation of equilibria from an extinction equilibrium which, by definition, is an equilibrium $(\hat{x}, \hat{u})=(\hat{0}, \hat{u})$ of $(6)$. From $(6 \mathrm{~b})$ we find that $\left(\hat{0}, \hat{u}^{*}\right)$ is an equilibrium if an only if

$$
\nabla_{\hat{v}} r\left(\hat{0}, \hat{u}^{*}, \hat{u}^{*}\right)=\hat{0}_{n}
$$

(where $\hat{0}_{n}$ is the origin in $\mathbb{R}^{n}$ ), in which case we say $\hat{u}^{*}$ is a critical trait. As a bifurcation parameter we use the dominant eigenvalue of $P\left(\hat{0}, \hat{u}^{*}, \hat{u}^{*}\right)$, which we denote by

$$
r_{0}^{*}:=\rho\left[P\left(\hat{0}, \hat{u}^{*}, \hat{u}^{*}\right)\right] .
$$

This is the inherent growth rate of the population when the trait is held fixed at the critical trait $\hat{u}=\hat{u}^{*}$. As in the non-evolutionary case, we normalize the entries of the projection matrix so that

$$
P(\hat{x}, \hat{u}, \hat{v})=r_{0}^{*} Q(\hat{x}, \hat{u}, \hat{v})
$$

where $Q$ satisfies $\mathrm{H} 2$ and

$$
\rho\left[Q\left(\hat{0}, \hat{u}^{*}, \hat{u}^{*}\right)\right]=1
$$

Letting

$$
\bar{r}(\hat{x}, \hat{u}, \hat{v}):=\rho[Q(\hat{x}, \hat{u}, \hat{v})]
$$

we have

$$
r(\hat{x}, \hat{u}, \hat{v})=r_{0}^{*} \bar{r}(\hat{x}, \hat{u}, \hat{v}), \quad \bar{r}\left(\hat{0}, \hat{u}^{*}, \hat{u}^{*}\right)=1 .
$$

The Darwinian equations (6) are now

$$
\begin{aligned}
& \hat{x}(t+1)=r_{0}^{*} Q(\hat{x}(t), \hat{u}(t), \hat{u}(t)) \hat{x}(t) \\
& \hat{u}(t+1)=\hat{u}(t)+\frac{1}{\bar{r}(\hat{x}, \hat{u}, \hat{u})} M \nabla_{\hat{v}} \bar{r}(\hat{x}, \hat{u}, \hat{u}) .
\end{aligned}
$$

Note that the bifurcation parameter $r_{0}^{*}$ does not appear in the trait equation (10b). This is because in the trait equation $(6 \mathrm{~b})$ we have

$$
\nabla_{\hat{v}} \ln r(\hat{x}(t), \hat{u}(t), \hat{u}(t))=\frac{1}{r(\hat{x}, \hat{u}, \hat{u})} \nabla_{\hat{v}} r(\hat{x}, \hat{u}, \hat{u})=\frac{1}{r_{0}^{*} \bar{r}(\hat{x}, \hat{u}, \hat{u})} r_{0}^{*} \nabla_{\hat{v}} \bar{r}(\hat{x}, \hat{u}, \hat{u})
$$


in which $r_{0}^{*}$ cancels.

We say that a pair $\left(r_{0}^{*},(\hat{x}, \hat{u})\right) \in \mathbb{R} \times(\Omega \times V)$ is an equilibrium pair if

$$
\begin{aligned}
\hat{x} & =r_{0}^{*} Q(\hat{x}, \hat{u}, \hat{u}) \hat{x} \\
\hat{0}_{n} & =\nabla_{\hat{v}} \bar{r}(\hat{x}, \hat{u}, \hat{u}) .
\end{aligned}
$$

Note that

$$
\nabla_{\hat{v}} \bar{r}(\hat{x}, \hat{u}, \hat{u})=\hat{0}_{n} \text { if and only if } \nabla_{\hat{v}} r(\hat{x}, \hat{u}, \hat{u})=\hat{0}_{n} .
$$

Definition 2 We say an equilibrium pair $\left(r_{0}^{*},(\hat{x}, \hat{u})\right)$ is a positive equilibrium if $\hat{x} \in \mathbb{R}_{+}^{m}$. An extinction equilibrium pair is an equilibrium pair of the form $\left(r_{0}^{*},(\hat{0}, \hat{u})\right)$.

Observe that $\left(r_{0}^{*},(\hat{0}, \hat{u})\right)$ is an extinction equilibrium pair if and only if $\hat{u}=\hat{u}^{*}$ is a critical trait and, conversely, if $\hat{u}=\hat{u}^{*}$ is a critical trait, then $\left(r_{0}^{*},\left(\hat{0}, \hat{u}^{*}\right)\right)$ is an extinction equilibrium pair for all values of $r_{0}^{*}$.

\section{Stability of extinction equilibria}

We want to analyze the stability properties of an extinction equilibrium pair.

Definition 3 We say that an equilibrium pair $\left(r_{0}^{*},(\hat{x}, \hat{u})\right)$ is stable if $(\hat{x}, \hat{u})$ is (locally asymptotically) stable as an equilibrium of the Darwinian dynamics (6).

To use the Linearization Principle, we compute the Jacobian matrix for the system (10a)-(10b)

$$
\mathcal{J}\left(r_{0}^{*}, \hat{x}, \hat{u}\right)=\left(\begin{array}{cc}
r_{0}^{*} J(\hat{x}, \hat{u}, \hat{u}) & r_{0}^{*} \Psi(\hat{x}, \hat{u}, \hat{u}) \\
\Upsilon(\hat{x}, \hat{u}, \hat{u}) & \Phi(\hat{x}, \hat{u}, \hat{u})
\end{array}\right)
$$

where $J(\hat{x}, \hat{u}, \hat{u})$ is the $m \times m$ Jacobian matrix of $Q(\hat{x}, \hat{u}, \hat{u}) \hat{x}$ with respect to $\hat{x}$ and $\Psi(\hat{x}, \hat{u}, \hat{u})$ is the $m \times n$ matrix whose $n$ columns are

$$
\partial_{u_{i}} Q(\hat{x}, \hat{u}, \hat{u}) \hat{x}+\partial_{v_{i}} Q(\hat{x}, \hat{u}, \hat{u}) \hat{x}, \quad i=1,2, \cdots, n .
$$

The dynamics of the $i^{\text {th }}$ mean trait $u_{i}$ are given by

$$
u_{i}(t+1)=u_{i}(t)+\sum_{k=1}^{n} \sigma_{i k} \partial_{v_{k}} \ln \bar{r}(\hat{x}, \hat{u}, \hat{u})
$$

and therefore $\Upsilon(\hat{x}, \hat{u}, \hat{u})$ is the $n \times m$ matrix whose $i^{t h}$ row $(i=1,2, \cdots, n)$ is the transpose of the gradient

$$
\nabla_{\hat{x}} \sum_{k=1}^{n} \sigma_{i k} \partial_{v_{k}} \ln \bar{r}(\hat{x}, \hat{u}, \hat{u})=\left.\sum_{k=1}^{n} \sigma_{i k} \nabla_{\hat{x}} \partial_{v_{k}} \ln \bar{r}(\hat{x}, \hat{u}, \hat{v})\right|_{\hat{v}=\hat{u}}
$$

and

$$
\Phi(\hat{x}, \hat{u}, \hat{u})=I_{n \times n}+M H(\hat{x}, \hat{u}, \hat{u})
$$

where $H(\hat{x}, \hat{u}, \hat{u})$ is a $n \times n$ matrix whose $k j^{t h}$ entry is the $u_{k}$ derivative of $\partial_{v_{j}} \ln \bar{r}(\hat{x}, \hat{u}, \hat{u})$, i.e.

$$
H(\hat{x}, \hat{u}, \hat{u}):=\left[\left.\partial_{u_{k} v_{j}} \ln \bar{r}(\hat{x}, \hat{u}, \hat{v})\right|_{\hat{v}=\hat{u}}+\left.\partial_{v_{k} v_{j}} \ln \bar{r}(\hat{x}, \hat{u}, \hat{v})\right|_{\hat{v}=\hat{u}}\right]
$$


By assumption H2, the projection matrix $P(\hat{0}, \hat{u}, \hat{v})=\left[\tilde{p}_{i j}(\hat{v})\right]$ is independent of $\hat{u}$ and as a result

$$
\left.\partial_{u_{k} v_{j}} \ln \bar{r}(\hat{x}, \hat{u}, \hat{v})\right|_{(\hat{x}, \hat{u}, \hat{v})=(\hat{0}, \hat{u}, \hat{u})} \equiv 0
$$

for all $\hat{u}$. It follows that

$$
H\left(\hat{0}, \hat{u}^{*}, \hat{u}^{*}\right)=\left[\partial_{v_{k} v_{j}}^{0} \ln \bar{r}\right]
$$

where we have adopted the superscript notation

$$
\partial_{v_{k} v_{j}}^{0} \ln \bar{r}:=\left.\partial_{v_{k} v_{j}} \ln \bar{r}(\hat{x}, \hat{u}, \hat{v})\right|_{(\hat{x}, \hat{u}, \hat{v})=\left(\hat{0}, \hat{u}^{*}, \hat{u}^{*}\right)} .
$$

Thus, the matrix $H\left(\hat{0}, \hat{u}^{*}, \hat{u}^{*}\right)$ is the Hessian of $\ln \bar{r}(\hat{x}, \hat{u}, \hat{v})$ with respect to $\hat{v}$ evaluated at $(\hat{x}, \hat{u}, \hat{v})=$ $\left(\hat{0}, \hat{u}^{*}, \hat{u}^{*}\right)$.

The Jacobian $\mathcal{J}$ evaluated at an extinction equilibrium pair $\left(r_{0}^{*},(\hat{x}, \hat{u})\right)=\left(r_{0}^{*},\left(\hat{0}, \hat{u}^{*}\right)\right)$ is

$$
\mathcal{J}\left(r_{0}^{*}, \hat{0}, \hat{u}^{*}\right)=\left(\begin{array}{cc}
r_{0}^{*} J\left(\hat{0}, \hat{u}^{*}, \hat{u}^{*}\right) & 0_{m \times n} \\
\Upsilon\left(\hat{0}, \hat{u}^{*}, \hat{u}^{*}\right) & \Phi\left(\hat{0}, \hat{u}^{*}, \hat{u}^{*}\right)
\end{array}\right)=\left(\begin{array}{cc}
r_{0}^{*} Q\left(\hat{0}, \hat{u}^{*}, \hat{u}^{*}\right) & 0_{m \times n} \\
\Upsilon\left(\hat{0}, \hat{u}^{*}, \hat{u}^{*}\right) & \Phi\left(\hat{0}, \hat{u}^{*}, \hat{u}^{*}\right)
\end{array}\right)
$$

where $0_{m \times n}$ denotes the null matrix with dimension $m \times n$. We note that $J\left(\hat{0}, \hat{u}^{*}, \hat{u}^{*}\right)$ is the Jacobian with respect to $\hat{x}$ of (10a) when the trait is held fixed at $\hat{u}^{*}$. The eigenvalues of (15) are the $m$ eigenvalues of $r_{0}^{*} Q\left(\hat{0}, \hat{u}^{*}, \hat{u}^{*}\right)$ and the $n$ eigenvalues of $\Phi\left(\hat{0}, \hat{u}^{*}, \hat{u}^{*}\right)$.

Recall that $r_{0}^{*}$ is the (strictly) dominant eigenvalue of $r_{0}^{*} Q\left(\hat{0}, \hat{u}^{*}, \hat{u}^{*}\right)$. Thus, if $r_{0}^{*}>1$ the extinction equilibrium $\left(\hat{0}, \hat{u}^{*}\right)$ is unstable. On the other hand, if $r_{0}^{*}<1$ then stability (by linearization) is determined by the $n$ eigenvalues of $\Phi\left(\hat{0}, \hat{u}^{*}, \hat{u}^{*}\right)$. Using the linearization principle for discrete dynamical systems [14], we obtain the following result, which is an extension, for the evolutionary case with multiple traits, of the first statement in Theorem 1.

Theorem 2 Assume H2 holds and that $\hat{u}^{*} \in V$ is a critical trait.

(a) If $r_{0}^{*}<1$ and $\rho\left[\Phi\left(\hat{0}, \hat{u}^{*}, \hat{u}^{*}\right)\right]<1$, then the extinction equilibrium pair $\left(r_{0}^{*},\left(\hat{0}, \hat{u}^{*}\right)\right)$ is stable.

(b) If $r_{0}^{*}<1$ and $\rho\left[\Phi\left(\hat{0}, \hat{u}^{*}, \hat{u}^{*}\right)\right]>1$, then the extinction equilibrium pair $\left(r_{0}^{*},\left(\hat{0}, \hat{u}^{*}\right)\right)$ is unstable.

(c) If $r_{0}^{*}>1$, then the extinction equilibrium pair $\left(r_{0}^{*},\left(\hat{0}, \hat{u}^{*}\right)\right)$ is unstable.

To investigate the spectral radius $\rho\left[\Phi\left(\hat{0}, \hat{u}^{*}, \hat{u}^{*}\right)\right]$, which appears in Theorem 2, we make further assumptions on the matrix $M$.

H3. The variance-covariance matrix $M$ diagonally dominant: $\sigma_{i}^{2} \geq \sum_{j \neq i}\left|\sigma_{i j}\right|$.

In [27] it is shown, under assumption H3, that $\rho\left[\Phi\left(\hat{0}, \hat{u}^{*}, \hat{u}^{*}\right)\right]<1$ if $H\left(\hat{0}, \hat{u}^{*}, \hat{u}^{*}\right)$ is negative definite and that $\rho\left[\Phi\left(\hat{0}, \hat{u}^{*}, \hat{u}^{*}\right)\right]>1$ if $H\left(\hat{0}, \hat{u}^{*}, \hat{u}^{*}\right)$ is positive semi-definite or indefinite provided the variances $\sigma_{i}^{2}$ are small.

Corollary 1 Assume H2 and H3 hold and that $\hat{u}^{*} \in V$ is a critical trait. If the variances $\sigma_{i}^{2}$ are small, then the extinction equilibrium pair $\left(r_{0}^{*},\left(\hat{0}, \hat{u}^{*}\right)\right)$ is

(a) stable if $r_{0}^{*}<1$ and the Hessian (14) is negative definite;

(b) unstable if $r_{0}^{*}>1$ or if the Hessian (14) is either indefinite or positive semi-definite. 
With regard to the variances, the assumption $\sigma_{i}^{2}<1 / \rho\left[H\left(\hat{0}, \hat{u}^{*}, \hat{u}^{*}\right)\right]$ is sufficient in Corollary 1. We are particularly interested in the case when the extinction equilibrium $\left(r_{0}^{*},\left(\hat{0}, \hat{u}^{*}\right)\right)$ loses stability as $r_{0}^{*}$ increases through 1 . This occurs in Theorem 2 when $\rho\left[\Phi\left(\hat{0}, \hat{u}^{*}, \hat{u}^{*}\right)\right]<1$. It also occurs in Corollary 1 , when $H\left(\hat{0}, \hat{u}^{*}, \hat{u}^{*}\right)$ is negative definite. This suggests the possibility that the (transcritical) bifurcation occurring at $r_{0}^{*}=1$ can result in a branch of stable positive (non-extinction) equilibria. We address this question in Section 5.

As an example consider the case when there is no covariant evolution of the traits (i.e. that the off diagonal terms in $M$ are all equal to 0 and the diagonal terms $\sigma_{i}^{2}$ are positive) and when

$$
\partial_{v_{i} v_{j}}^{0} \bar{r}=0 \text { for } i \neq j \text {, }
$$

so that traits evolve nearly independently in a neighborhood of $(\hat{x}, \hat{u})=\left(\hat{0}, \hat{u}^{*}\right)$. With these assumptions the matrix $\Phi\left(\hat{0}, \hat{u}^{*}, \hat{u}^{*}\right)$ is diagonal and its eigenvalues are $1+\sigma_{i}^{2} \partial_{v_{i} v_{i}}^{0} \bar{r}$. From Theorem 2 we obtain the following corollary.

Corollary 2 Assume H2 holds and that $\hat{u}^{*} \in V$ is a critical trait. Further assume $\sigma_{i j}=0$ and (16) for all $i \neq j$. The extinction equilibrium pair $\left(r_{0}^{*},\left(\hat{0}, \hat{u}^{*}\right)\right)$ is

(a) stable if $r_{0}^{*}<1$ and $\left|1+\sigma_{i}^{2} \partial_{v_{i} v_{i}}^{0} \bar{r}\right|<1$ for all $i$;

(b) unstable if $r_{0}^{*}>1$;

(c) unstable for any $r_{0}^{*}>0$ if $\left|1+\sigma_{i}^{2} \partial_{v_{i} v_{i}}^{0} \bar{r}\right|>1$ for at least one $i$.

Note. In Corollary 2(c) the extinction equilibrium pair $\left(r_{0}^{*},\left(\hat{0}, \hat{u}^{*}\right)\right)$ is unstable, for any value of $r_{0}^{*}$, if $\partial_{v_{i} v_{i}}^{0} \bar{r}>0$ for at least one $i$. On the other hand, if $\partial_{v_{i} v_{i}}^{0} \bar{r}<0$ for all values of $i$, then extinction equilibrium pair $\left(r_{0}^{*},\left(\hat{0}, \hat{u}^{*}\right)\right)$ is stable for $r_{0}^{*}<1$ and small variances $\sigma_{i}^{2}$.

\section{A Bifurcation Theorem for the Evolutionary Model (10)}

The loss of stability by the extinction equilibrium pair when $r_{0}^{*}$ increases through 1 suggests the possibility of a (transcritical) bifurcation at the value $r_{0}^{*}=1$. This is due to the fact that an eigenvalue of the Jacobian leaves the complex unit circle as $r_{0}^{*}$ increases through 1 . In this section we establish a bifurcation theorem for the evolutionary model (10).

We begin by assuming that $\hat{u}$ can be expressed as a function of $\hat{x}$ by means of the equilibrium equation (11b).

H4. Let $\hat{u}^{*} \in V$ be a critical trait. Assume there exists a function $\hat{\xi} \in C^{2}(N \rightarrow V)$, where $N$ is a open neighborhood of $\hat{0}$ in $\mathbb{R}^{m}$, such that $\hat{\xi}(\hat{0})=\hat{u}^{*}$ and $\nabla_{\hat{v}} \bar{r}(\hat{x}, \hat{\xi}(\hat{x}), \hat{\xi}(\hat{x}))=$ $\hat{0}_{n}$ for $\hat{x} \in N$.

Let $J_{\hat{u}}^{0}\left(\nabla_{\hat{v}} \bar{r}\right)$ and $J_{\hat{x}}^{0}\left(\nabla_{\hat{v}} \bar{r}\right)$ denote the Jacobian matrices of the gradient $\nabla_{\hat{v}} \bar{r}(\hat{x}, \hat{u}, \hat{u})$ with respect to $\hat{u}$ and $\hat{x}$ respectively evaluated at $(\hat{x}, \hat{u}, \hat{u})=\left(\hat{0}, \hat{u}^{*}, \hat{u}^{*}\right)$. The following assumption and the Implicit Function Theorem guarantee that H4 holds.

H5. Let $u^{*} \in V$ be a critical trait for which $J_{\hat{u}}^{0}\left(\nabla_{\hat{v}} \bar{r}\right)$ is a non-singular matrix.

Remark 4 The product rule (31) applied to

$$
\left.\nabla_{\hat{v}} \ln \bar{r}(\hat{x}, \hat{u}, \hat{v})\right|_{\hat{v}=\hat{u}}=\left.\frac{1}{\bar{r}} \nabla_{\hat{v}} \bar{r}(\hat{x}, \hat{u}, \hat{v})\right|_{\hat{v}=\hat{u}}
$$


evaluated at $\hat{x}=\hat{0}$ implies, together with $\bar{r}^{0}=1$ and $\nabla_{\hat{v}}^{0} \bar{r}=\hat{0}$ (by the definitikon of a critical trait), that $J_{\hat{u}}^{0} \nabla_{\hat{v}} \ln \bar{r}=J_{\hat{u}}^{0} \nabla_{\hat{v}} \bar{r}$ or

$$
J_{\hat{u}}^{0} \nabla_{\hat{v}} \ln \bar{r}=\left[\partial_{v_{k} v_{j}}^{0} \ln \bar{r}\right] .
$$

Thus, under assumption H2 we see (from (14)) that in $\mathrm{H} 5$

$$
J_{\hat{u}}^{0}\left(\nabla_{\hat{v}} \bar{r}\right)=H\left(\hat{0}, \hat{u}^{*}, \hat{u}^{*}\right) .
$$

Theorem 3 Assume $\hat{u}^{*} \in V$ is a critical trait. Assume H2 and $H_{4}$ hold and that $\kappa^{*} \neq 0$.

(1) There exists a continuum $\mathcal{C}^{*}$ of positive equilibrium pairs $\left(r_{0}^{*},(\hat{x}, \hat{u})\right) \in \mathbb{R}_{+} \times\left(\mathbb{R}_{+}^{m} \times V\right)$ of (10) that bifurcates from the extinction pair $\left(1,\left(\hat{0}, \hat{u}^{*}\right)\right)$ (i.e. that contains the extinction pair in its closure).

(2) Assume H2 and H5 hold. In a neighborhood of $\left(1,\left(\hat{0}, \hat{u}^{*}\right)\right)$, the positive equilibrium pairs have the parametric representation

$$
\begin{aligned}
\hat{x}(\varepsilon) & =\hat{w}_{R} \varepsilon+\mathcal{O}\left(\varepsilon^{2}\right) \\
\hat{u}(\varepsilon) & =\hat{u}^{*}+\hat{u}_{1} \varepsilon+\mathcal{O}\left(\varepsilon^{2}\right) \\
r_{0}^{*}(\varepsilon) & =1+\kappa^{*} \varepsilon+\mathcal{O}\left(\varepsilon^{2}\right)
\end{aligned}
$$

for small $\varepsilon \gtrsim 0$ where $\hat{w}_{R}$ is a positive right eigenvector of $Q\left(\hat{0}, \hat{u}^{*}, \hat{u}^{*}\right)$ associated with eigenvalue 1 (equivalently of $P\left(\hat{0}, \hat{u}^{*}, \hat{u}^{*}\right)$ when $r_{0}^{*}=1$ ) and

$$
\begin{aligned}
\hat{u}_{1} & :=-\left[J_{\hat{u}}^{0}\left(\nabla_{\hat{v}} \bar{r}\right)\right]^{-1} J_{\hat{x}}^{0}\left(\nabla_{\hat{v}} \bar{r}\right) \hat{w}_{R} . \\
\kappa^{*} & :=-\hat{w}_{L}^{T}\left(\left[\nabla_{\hat{x}}^{0} q_{i j}^{T} \hat{w}_{R}\right]\right) \hat{w}_{R} .
\end{aligned}
$$

Furthermore, we have the following alternatives.

(a) If $\rho\left[\Phi\left(\hat{0}, \hat{u}^{*}, \hat{u}^{*}\right)\right]<1$ and $\kappa^{*}>0$, then the bifurcation of $\mathcal{C}^{*}$ is forward and the positive equilibrium pairs on $\mathcal{C}^{*}$ are stable.

(b) If $\rho\left[\Phi\left(\hat{0}, \hat{u}^{*}, \hat{u}^{*}\right)\right]<1$ and $\kappa^{*}<0$, then the bifurcation is backward and the positive equilibrium pairs on $\mathcal{C}^{*}$ are unstable.

(c) If $\rho\left[\Phi\left(\hat{0}, \hat{u}^{*}, \hat{u}^{*}\right)\right]>1$, then positive equilibrium pairs in the continuum $\mathcal{C}^{*}$ are unstable regardless of the direction of bifurcation.

Remark 5 Because $\kappa^{*}$ is calculated from evaluations at the bifurcation point $\left(r_{0}^{*},(\hat{x}, \hat{u})\right)=\left(1,\left(\hat{0}, \hat{u}^{*}\right)\right)$ and because only the sign of $\kappa^{*}$ is involved in determining the direction of bifurcation and stability, Theorem 3, parts (a) and (b), remains valid if in the formula (19) and in $\mathrm{H}_{4}$ and H5 we replace $q_{i j}$ by $p_{i j}$ and $\bar{r}$ by $r$.

Proof. (1) Under H4, the equilibrium equations (11) reduce to the single equation

$$
\hat{x}=r_{0}^{*} Q(\hat{x}, \hat{\xi}(\hat{x}), \hat{\xi}(\hat{x})) \hat{x}
$$

for $\hat{x} \in N$. Theorem 1 applies to this equation with matrix $Q(\hat{x}, \hat{\xi}(\hat{x}), \hat{\xi}(\hat{x}))$ in place of $Q(\hat{x})$ (and $N$ in place of $\Omega$ ). This results in the existence of a continuum $\mathcal{C}$ of positive solution pairs $\left(r_{0}^{*}, \hat{x}\right)$ of $(20)$ that bifurcates from $(1, \hat{0})$. The continuum $\mathcal{C}$ in turn gives rise to a continuum

$$
\mathcal{C}^{*}:=\left\{\left(r_{0}^{*},(\hat{x}, \hat{u})\right) \mid\left(r_{0}^{*}, \hat{x}\right) \in \mathcal{C}, \hat{u}=\hat{\xi}(\hat{x})\right\}
$$


of equilibrium pairs $\left(r_{0}^{*},(\hat{x}, \hat{u})\right)$ of $(10)$ that bifurcates from the extinction equilibrium $\left(1,\left(\hat{0}, \hat{u}^{*}\right)\right)$ at $r_{0}^{*}=1$

(2) The parameterization of $\mathcal{C}$ Theorem 1 implies that, near the bifurcation point, the positive equilibrium pairs on the continuum $\mathrm{C}^{*}$ have a parameterization (17). The coefficient $\kappa^{*}$ is given by the formula (3) for $\kappa$ but with $p_{i j}(\hat{x})$ replaced by $p_{i j}(\hat{x}, \hat{\xi}(\hat{x}), \hat{\xi}(\hat{x}))$. To make this calculation we note that the coefficient $\hat{u}_{1}=\nabla^{0} \hat{\xi}^{T} \hat{w}_{R}$ can be calculated by an implicit differentiation of the equation $\nabla_{\hat{v}} \bar{r}(\hat{x}(\varepsilon), \hat{\xi}(\hat{x}(\varepsilon)), \hat{\xi}(\hat{x}(\varepsilon)))=\hat{0}_{n}$ with respect to $\varepsilon$ and a subsequent evaluation at $\varepsilon=0$. From this calculation we obtain (18). From (3) and Lemma 1 in the Appendix, but with $p_{i j}(\hat{x})$ replaced by $p_{i j}(\hat{x}, \hat{\xi}(\hat{x}), \hat{\xi}(\hat{x}))$, we obtain (19).

To investigate the stability of bifurcating positive equilibrium pairs we make use of the parametrization (17) which allows us to parameterize by $\varepsilon$ the Jacobian $\mathcal{J}(r, \hat{x}, \hat{u})$ when it is evaluated at the equilibria (17) and, subsequently, to parameterize this Jacobian's eigenvalues by $\varepsilon$. From this parameterization we can approximate the eigenvalues of the Jacobian for $\varepsilon \gtrsim 0$.

At $\varepsilon=0$ the eigenvalues of the Jacobian $\mathcal{J}\left(1, \hat{0}, \hat{u}^{*}\right)$ are the eigenvalues of $J\left(\hat{0}, \hat{u}^{*}, \hat{u}^{*}\right)$ and the eigenvalues of $\Phi\left(\hat{0}, \hat{u}^{*}, \hat{u}^{*}\right)$. The spectrum of the Jacobian $\mathcal{J}\left(r_{0}^{*}(\varepsilon), \hat{x}(\varepsilon), \hat{u}(\varepsilon)\right)$ approaches, by continuity, the spectrum of $\mathcal{J}\left(1, \hat{0}, \hat{u}^{*}\right)$ as $\varepsilon$ tends to 0 . Therefore, if $\rho\left[\Phi\left(\hat{0}, \hat{u}^{*}, \hat{u}^{*}\right)\right]>1$ then for $\varepsilon \gtrsim 0$ the Jacobian $\mathcal{J}\left(r_{0}^{*}(\varepsilon), \hat{x}(\varepsilon), u(\varepsilon)\right)$ also has spectral radius greater than 1 . Consequently, the positive equilibria are unstable near the bifurcation point. This establishes 2(c).

Suppose $\rho\left[\Phi\left(\hat{0}, \hat{u}^{*}, \hat{u}^{*}\right)\right]<1$. Since the strictly dominant eigenvalue of $J\left(\hat{0}, \hat{u}^{*}, \hat{u}^{*}\right)$ is 1 , it follows that the dominant eigenvalue of $\mathcal{J}\left(1, \hat{0}, u^{*}\right)$ is 1 . To determine stability of the bifurcating positive equilibria, by means of the linearization principle, we must investigate if the dominant eigenvalue of $\mathcal{J}\left(r_{0}^{*}(\varepsilon), \hat{x}(\varepsilon), \hat{u}(\varepsilon)\right)$, which equals 1 when $\varepsilon=0$, is greater or less than 1 for $\varepsilon \gtrsim 0$. Let

$$
\mu(\varepsilon)=1+\mu_{1} \varepsilon+\mathcal{O}\left(\varepsilon^{2}\right)
$$

denote the dominant eigenvalue of $\mathcal{J}\left(r_{0}^{*}(\varepsilon), \hat{x}(\varepsilon), \hat{u}(\varepsilon)\right)$. Whether $\mu(\varepsilon)$ is less than or greater than 1 for $\varepsilon \gtrsim 0$, and hence whether the bifurcating positive equilibria are stable or unstable near the bifurcation point, can be determined by the sign of $\mu_{1}$ : for $\varepsilon \gtrsim 0$, the bifurcating positive equilibria are stable if $\mu_{1}<0$ and unstable if $\mu_{1}>0$.

To calculate a formula for $\mu_{1}$ we begin by letting

$$
\hat{W}_{R}(\varepsilon)=\hat{W}_{R 0}+\hat{W}_{R 1} \varepsilon+\mathcal{O}\left(\varepsilon^{2}\right)
$$

denote a right eigenvector of the Jacobian $\mathcal{J}$ associated with the dominant eigenvalue $\mu(\varepsilon)$, so that

$$
\mathcal{J}\left(r_{0}^{*}(\varepsilon), \hat{x}(\varepsilon), \hat{u}(\varepsilon)\right) \hat{W}_{R}(\varepsilon)=\mu(\varepsilon) \hat{W}_{R}(\varepsilon) .
$$

Setting $\varepsilon=0$ in $(21)$ we obtain

$$
\mathcal{J}\left(1, \hat{0}, \hat{u}^{*}\right) \hat{W}_{R 0}=\hat{W}_{R 0}
$$

We can write

$$
\hat{W}_{R 0}=\left(\begin{array}{c}
\hat{w}_{R 0}^{m} \\
\hat{w}_{R 0}^{n}
\end{array}\right)
$$

where $\hat{w}_{R 0}^{m}$ and $\hat{w}_{R 0}^{n}$, are column vectors with $m$ and $n$ entries respectively. The vector $\hat{w}_{R 0}^{m}$ is a right eigenvector of $J\left(\hat{0}, \hat{u}^{*}, \hat{u}^{*}\right)=Q\left(\hat{0}, \hat{u}^{*}, \hat{u}^{*}\right)$ associated with the eigenvalue 1 and consequently $\hat{w}_{R 0}^{m}=\hat{w}_{R}$. A calculation shows

$$
\hat{W}_{R 0}=\left(\begin{array}{c}
\hat{w}_{R} \\
\hat{u}_{1}
\end{array}\right) .
$$


The vector $\hat{W}_{L 0}^{T}$ where

$$
\hat{W}_{L 0}=\left(\begin{array}{c}
\hat{w}_{L} \\
\hat{0}_{n}
\end{array}\right)
$$

is a left eigenvector of $\mathcal{J}\left(1, \hat{0}, \hat{u}^{*}\right)$ (where $\hat{w}_{L}^{T}$ is a left eigenvector of $J\left(\hat{0}, \hat{u}^{*}, \hat{u}^{*}\right)$ ). Note that $\hat{W}_{L 0}^{T} \hat{W}_{R 0}=\hat{w}_{L}^{T} \hat{w}_{R}=1$.

If we differentiate (21) with respect to $\varepsilon$ and set $\varepsilon=0$ in the result, we obtain

$$
\left(\mathcal{J}\left(1, \hat{0}, \hat{u}^{*}\right)-I_{m+n}\right) \hat{W}_{R 1}=\mu_{1} \hat{W}_{R 0}-\left.\frac{d}{d \varepsilon} \mathcal{J}\left(r_{0}^{*}(\varepsilon), \hat{x}(\varepsilon), \hat{u}(\varepsilon)\right)\right|_{\varepsilon=0} \hat{W}_{R 0}
$$

where $I_{m+n}$ denotes the identity matrix of size $m+n$. According to Fredholm alternative, this equation is solvable for $\hat{W}_{R 1}$ if and only if the right hand side is orthogonal to the kernel of $\left(\mathcal{J}\left(1, \hat{0}, \hat{u}^{*}\right)-I_{m+n}\right)^{T}$. This kernel is spanned by the left eigenvectors of $\mathcal{J}\left(1, \hat{0}, \hat{u}^{*}\right)$ associated with the eigenvalue 1 . Therefore, the right hand side must be orthogonal to $\hat{W}_{L 0}$. This implies

$$
\begin{gathered}
\mathcal{J}\left(r_{0}^{*}, \hat{x}, \hat{u}\right)=\left(\begin{array}{cc}
r_{0}^{*} J(\hat{x}, \hat{u}, \hat{u}) & r_{0}^{*} \Psi(\hat{x}, \hat{u}, \hat{u}) \\
\Upsilon(\hat{x}, \hat{u}, \hat{u}) & \Phi(\hat{x}, \hat{u}, \hat{u})
\end{array}\right) \\
\mu_{1}=\left.\hat{W}_{L 0}^{T} \frac{d}{d \varepsilon} \mathcal{J}\left(r_{0}^{*}(\varepsilon), \hat{x}(\varepsilon), \hat{u}(\varepsilon)\right)\right|_{\varepsilon=0} \hat{W}_{R 0} \\
=\left.\left(\begin{array}{c}
\hat{w}_{L} \\
\hat{0}_{n}
\end{array}\right)^{T} \frac{d}{d \varepsilon}\left(\begin{array}{cc}
r_{0}^{*}(\varepsilon) J(\hat{x}(\varepsilon), \hat{u}(\varepsilon), \hat{u}(\varepsilon)) & r_{0}^{*}(\varepsilon) \Psi(\hat{x}(\varepsilon), \hat{u}(\varepsilon), \hat{u}(\varepsilon)) \\
\Upsilon(\hat{x}(\varepsilon), \hat{u}(\varepsilon), \hat{u}(\varepsilon)) & \Phi(\hat{x}(\varepsilon), \hat{u}(\varepsilon), \hat{u}(\varepsilon))
\end{array}\right)\right|_{\varepsilon=0}\left(\begin{array}{c}
\hat{w}_{R} \\
\hat{u}_{1}
\end{array}\right)
\end{gathered}
$$

or

$$
\mu_{1}=\left.\hat{w}_{L}^{T} \frac{d}{d \varepsilon}\left[r_{0}^{*}(\varepsilon) J(\hat{x}(\varepsilon), \hat{u}(\varepsilon), \hat{u}(\varepsilon))\right]\right|_{\varepsilon=0} \hat{w}_{R}+\left.\hat{w}_{L}^{T} \frac{d}{d \varepsilon}\left[r_{0}^{*}(\varepsilon) \Psi(\hat{x}(\varepsilon), \hat{u}(\varepsilon), \hat{u}(\varepsilon))\right]\right|_{\varepsilon=0} \hat{u}_{1} .
$$

We consider the two terms in this sum one at a time. With regard to the first term in $\mu_{1}$, the product and chain rules imply

$$
\begin{aligned}
& \left.\hat{w}_{L}^{T} \frac{d}{d \varepsilon}\left[r_{0}^{*}(\varepsilon) J(\hat{x}(\varepsilon), \hat{u}(\varepsilon), \hat{u}(\varepsilon))\right]\right|_{\varepsilon=0} \hat{w}_{R} \\
= & \kappa^{*} \hat{w}_{L}^{T} Q\left(\hat{0}, \hat{u}^{*}, \hat{u}^{*}\right) \hat{w}_{R}+\left.\hat{w}_{L}^{T} \frac{d}{d \varepsilon} J(\hat{x}(\varepsilon), \hat{u}(\varepsilon), \hat{u}(\varepsilon))\right|_{\varepsilon=0} \hat{w}_{R} \\
= & \kappa^{*}+\hat{w}_{L}^{T}\left(\left[\nabla_{\hat{x}}^{0} q_{i j}^{T} \hat{w}_{R}\right]+\left[\nabla_{\hat{u}}^{0} q_{i j}^{T} \hat{u}_{1}\right]+\left[\nabla_{\hat{v}}^{0} q_{i j}^{T} \hat{u}_{1}\right]+\left[\partial_{x_{i}}^{0} q_{j} \hat{w}_{R}\right]\right) \hat{w}_{R}
\end{aligned}
$$

where we have defined the row vector

$$
\partial_{x_{i}}^{0} q_{j}:=\left[\begin{array}{llll}
\partial_{x_{i}}^{0} q_{j 1} & \partial_{x_{i}}^{0} q_{j 2} & \cdots & \partial_{x_{i}}^{0} q_{j m}
\end{array}\right] .
$$

With regard to the second term in $\mu_{1}$, we recall that $\Psi(\hat{x}, \hat{u}, \hat{u})$ is the $m \times n$ matrix whose $n$ columns are (12) and as a result, upon evaluation at the bifurcation point, the only contribution to the derivative in the second term arises from the derivatives 
of $\Psi(\hat{x}, \hat{u}, \hat{u})=\left[\psi_{i j}(\hat{x}, \hat{u}, \hat{u})\right]$ with respect to the components of $\hat{x}$. Therefore the second term in $\mu_{1}$ is

$$
\begin{aligned}
\left.\hat{w}_{L}^{T} \frac{d}{d \varepsilon}\left[r_{0}^{*}(\varepsilon) \Psi(\hat{x}(\varepsilon), \hat{u}(\varepsilon), \hat{u}(\varepsilon))\right]\right|_{\varepsilon=0} \hat{u}_{1} & =\hat{w}_{L}^{T}\left[\nabla_{\hat{x}}^{0} \psi_{i j}^{T} \hat{w}_{R}\right] \hat{u}_{1} \\
& =\hat{w}_{L}^{T}\left(\left[\partial_{u_{i}}^{0} q_{j} \hat{w}_{R}\right]+\left[\partial_{v_{i}}^{0} q_{j} \hat{w}_{R}\right]\right) \hat{u}_{1}
\end{aligned}
$$

where we have defined the row vectors

$$
\partial_{u_{i}}^{0} q_{j}:=\left[\begin{array}{llll}
\partial_{u_{i}}^{0} q_{j 1} & \partial_{u_{i}}^{0} q_{j 2} & \cdots & \partial_{u_{i}}^{0} q_{j m}
\end{array}\right], \quad \partial_{v_{i}}^{0} q_{j}:=\left[\begin{array}{llll}
\partial_{v_{i}}^{0} q_{j 1} & \partial_{v_{i}}^{0} q_{j 2} & \cdots & \partial_{v_{i}}^{0} q_{j m}
\end{array}\right] .
$$

H2 implies $\partial_{u_{k}}^{0} q_{i j}=0$ for all $u_{k}$ and all $i, j$, and

$$
\mu_{1}=\kappa^{*}+\hat{w}_{L}^{T}\left[\nabla_{\hat{x}}^{0} q_{i j}^{T} \hat{w}_{R}\right] \hat{w}_{R}+\hat{w}_{L}^{T}\left[\nabla_{\hat{v}}^{0} q_{i j}^{T} \hat{u}_{1}\right] \hat{w}_{R}+\hat{w}_{L}^{T}\left[\partial_{x_{i}}^{0} q_{j} \hat{w}_{R}\right] \hat{w}_{R}+\hat{w}_{L}^{T}\left[\partial_{v_{i}}^{0} q_{j} \hat{w}_{R}\right] \hat{u}_{1} .
$$

\section{Noting that}

$$
\hat{w}_{L}^{T}\left[\nabla_{\hat{x}}^{0} q_{i j}^{T} \hat{w}_{R}\right] \hat{w}_{R}=\hat{w}_{L}^{T}\left[\partial_{x_{i}}^{0} q_{j} \hat{w}_{R}\right] \hat{w}_{R}, \quad \hat{w}_{L}^{T}\left[\nabla_{\hat{v}}^{0} q_{i j}^{T} \hat{u}_{1}\right] \hat{w}_{R}=\hat{w}_{L}^{T}\left[\partial_{v_{i}}^{0} q_{j} \hat{w}_{R}\right] \hat{u}_{1}
$$

we have

$$
\mu_{1}=\kappa^{*}+2\left(\hat{w}_{L}^{T}\left[\nabla_{\hat{x}}^{0} q_{i j}^{T} \hat{w}_{R}\right] \hat{w}_{R}+\hat{w}_{L}^{T}\left[\nabla_{\hat{v}}^{0} q_{i j}^{T} \hat{u}_{1}\right] \hat{w}_{R}\right) .
$$

By Lemma 1 in the Appendix we get

$$
\mu_{1}=\kappa^{*}+2 \hat{w}_{L}^{T}\left[\nabla_{\hat{x}}^{0} q_{i j}^{T} \hat{w}_{R}\right] \hat{w}_{R}
$$

which, by (18), implies $\mu_{1}=-\kappa^{*}$. As a result, $\kappa^{*}>0$ implies both that the bifurcation is forward and that the bifurcating positive equilibria are stable for $\varepsilon \gtrsim 0$. On the other hand, $\kappa^{*}<0$ implies that the bifurcation is backward and that the bifurcating positive equilibria are unstable for $\varepsilon \gtrsim 0$.

From (13) we obtain (as in [27]) the following corollary of Theorem 3.

Corollary 3 Assume H2, H3 and H5 hold and that $\hat{u}^{*} \in V$ is a critical trait. Let $\mathcal{C}^{*}$ be the continuum of positive equilibrium pairs that bifurcates from the extinction pair $\left(1,\left(\hat{0}, \hat{u}^{*}\right)\right)$ guaranteed by Theorem 3. If the variances $\sigma_{i}^{2}$ are small, then in a neighborhood of the bifurcation point $\left(1,\left(\hat{0}, \hat{u}^{*}\right)\right)$ we have the following alternatives.

(a) The bifurcation of $\mathcal{C}^{*}$ is forward and stable if the Hessian (14) is negative definite and $\kappa^{*}>0$.

(b) The bifurcation of $\mathcal{C}^{*}$ is backward and unstable if the Hessian (14) is negative definite and $\kappa^{*}<0$.

(c) The positive equilibrium pairs in the continuum $\mathcal{C}^{*}$ are unstable if the Hessian (14) is positive semi-definite or indefinite (regardless of the direction of bifurcation).

For the case of no trait covariance considered in Corollary 2, we obtain the following result from Corollary 3.

Corollary 4 Assume H2 and H5 hold and that $\hat{u}^{*} \in V$ is a critical trait. Further assume $\sigma_{i j}=0$ and (16) for all $i \neq j$. Let $\mathcal{C}^{*}$ be the continuum of positive equilibrium pairs that bifurcates from the extinction pair $\left(1,\left(\hat{0}, \hat{u}^{*}\right)\right)$ guaranteed by Theorem 3. Then in a neighborhood of the bifurcation point $\left(1,\left(\hat{0}, \hat{u}^{*}\right)\right)$ we have the following alternatives. 
(a) The bifurcation of $\mathcal{C}^{*}$ is forward and stable if $\kappa^{*}>0$ and $\left|1+\sigma_{i}^{2} \partial_{v_{i} v_{i}}^{0} \bar{r}\right|<1$ for all $i$.

(b) The bifurcation of $\mathcal{C}^{*}$ is backward and unstable if $\kappa^{*}<0$ and $\left|1+\sigma_{i}^{2} \partial_{v_{i} v_{i}}^{0} \bar{r}\right|<1$ for all $i$.

(c) The positive equilibrium pairs in the continuum $\mathcal{C}^{*}$ are unstable if $\left|1+\sigma_{i}^{2} \partial_{v_{i} v_{i}}^{0} \bar{r}\right|>1$ for at least one $i$ (regardless of the direction of bifurcation).

Note. In Corollary 4 we see that the positive equilibrium pairs in the continuum $\mathcal{C}^{*}$ are unstable if $\partial_{v_{i} v_{i}}^{0} \bar{r}>0$ for at least one $i$. On the other hand, if $\partial_{v_{i} v_{i}}^{0} \bar{r}<0$ for all values of $i$, then the positive equilibrium pairs in the continuum $\mathcal{C}^{*}$ are stable if $\kappa^{*}>0$ and the variances $\sigma_{i}^{2}$ are small enough so that $\left|1+\sigma_{i}^{2} \partial_{v_{i} v_{i}}^{0} \bar{r}\right|<1$, i.e. $\sigma_{i}^{2}<-2 / \partial_{v_{i} v_{i}}^{0} \bar{r}$.

\section{An Application}

Consider the single difference equation

$$
x(t+1)=b x(t) \exp (-c x(t)) \exp \left(-\frac{\alpha}{1+s x(t)}\right)
$$

with coefficients $b, c>0$ and $\alpha, s \geq 0$. When $\alpha=0$ this map is the famous Ricker equation which is one of the most well known equations that incorporates negative effects that population density can have on population growth. Equation (22) is studied in [25] as a model equation that incorporates a positive effect of increased population density (a so-called component Allee effect [5]) in the presence of a predator. This is the well-known predator-saturation effect in ecology and is one of the most commonly attributed causes of Allee effects [12], [5].

The factor $\exp (-\alpha /(1+s x))$ in $(22)$ is an increasing function of $x$ and represents the probability of escaping predation. We can interpret $\alpha$ as the intensity of predation and $s$ a measure of how effective the protection from predation attributed to population density $x$, which we will refer to as the predation protection factor. Re-writing (22) as

$$
x(t+1)=r_{0} \bar{r}(x) x(t)
$$

with

$$
r_{0}:=b \exp (-\alpha), \quad \bar{r}(x):=\exp \left(-c x(t)+\alpha \frac{s x(t)}{1+s x(t)}\right)
$$

we see that $r_{0}$ is the inherent (density-free) per capita birth rate, which equals $b$ in the absence of predation $\alpha=0$. This equation, studied in [25], not surprisingly can exhibit the same kind of period-doubling route-to-chaos as $r_{0}$ increases as does the famous Ricker equation when $\alpha=0$. (The right side of (23) defines a unimodal map.) The bifurcation that occurs at $r_{0}=1$ where the extinction equilibrium $x=0$ destabilizes is, according to Theorem 1, forward and therefore stable if $\kappa=c-\alpha s>0$. This inequality holds if the effect of predation, as measured by the product of the predation intensity $\alpha$ and (per capita) predation protection factor $s$, is small compared to that of the negative density effects measured by $c$. This occurs, of course, for the Ricker equation when $\alpha=0$.

On the other, if the reverse is true and the effect of predation $\alpha s$ is large compared to $c$, then by Theorem 1 the bifurcation at $r_{0}=1$ is backward and unstable. In this case, i.e. when $\alpha s>c$, we can also say some things about the bifurcating continuum $\mathcal{C}$ of positive equilibrium pairs $\left(r_{0}, x\right)$ outside the neighborhood of the bifurcation point $\left(r_{0}, x\right)=(1,0)$. The equation

$$
1=r_{0} \exp \left(-c x+\alpha \frac{s x}{1+s x}\right)
$$


satisfied by positive equilibria $x>0$, when re-written as

$$
r_{0}=\exp \left(c x-\alpha \frac{s x}{1+s x}\right)
$$

describes the continuum $\mathcal{C}$ of positive equilibrium pairs $\left(r_{0}, x\right)$. The graph of $r_{0}$ as a function of $x$ contains the point $r_{0}=1$ at $x=0$, decreases as $x$ increases to a unique critical point $x_{c r}>0$ at which $r_{0}$ attains a global minimum $r_{c r}>0$, and increases without bound for $x>x_{c r}$. See Figure 1. From the parabola-like shape of this graph, we see that the inverse function, treating $x$ as a function of $r_{0}$, has two branches: an upper branch of positive equilibria $x_{2}\left(r_{0}\right)$ for $r_{0} \geq r_{c r}$ and a lower branch of positive equilibria $x_{1}\left(r_{0}\right)<x_{2}\left(r_{0}\right)$ for $r^{c r} \leq r_{0}<1$ which satisfies $x_{1}(1)=0$. The value $r_{0}=r_{c r}$ is a saddle-node (blue-sky) bifurcation (or tipping) point at which the lower branch $x_{1}\left(r_{0}\right)$ and and upper branch $x_{2}\left(r_{0}\right)$ coalesce. The following facts follow from general results in [11]: for $r_{0}<r_{0}^{c r}$ the extinction equilibrium is globally asymptotically stable; the equilibria $x_{1}\left(r_{0}\right)$ are unstable and the equilibria $x_{2}\left(r_{0}\right)$ are (locally asymptotically) stable for $r_{0} \gtrsim r_{c r}$. The upper branch $x_{2}\left(r_{0}\right)$ might not remain stable for all $r_{0}>r_{c r}$, however, but might undergo a period doubling cascade to chaos. If a destabilization of $x_{2}\left(r_{0}\right)$ occurs at a point $r_{0} \geq 1$, then on the interval $r_{c r}<r_{0}<1$ there are two stable equilibria, the extinction equilibrium and the positive equilibrium $x_{2}\left(r_{0}\right)$. This scenario is called a strong Allee effect. It asserts that survival is possible for some $r_{0}<1$ provided a population's initial condition lies outside the basin of attractor of the extinction equilibrium (the Allee basin). If, on the other hand, $x_{2}\left(r_{0}\right)$ loses stability at a point in the interval $r_{c r}<r_{0}<1$, then there still occurs a strong Allee effect but one with a non-equilibrium survival attractor (e.g. a periodic cycle or a more complicated attractor).

Sample forward and backward bifurcation diagrams are shown in Figure 2. That secondary period-doubling bifurcations cascade to complex (presumably chaotic) dynamics in both cases is not unexpected, given that (23) is based on the Ricker nonlinearity. The backward bifurcation in Figure $2 \mathrm{~B}$ is an example illustrating a saddle-node bifurcation (at $r_{0} \approx 0.4$ ) that results a multi-stable equilibrium (strong Allee) scenario, as shown in Figure 1. In this example the positive equilibria destabilize (into a period doubling route to chaos) just outside the Allee interval $0.4<r_{0}<1$. In other examples, using different parameter values, this destabilization can occur at a value of $r_{0}<1$ so that the multi-attractor scenario of the strong Allee effect involves a stable cycle or even more complicated attractor. For examples and further results concerning the relationship between backward bifurcations and strong Allee effects, see [10]. The complex dynamics that can arise in this model, particularly when positive non-equilibrium attractors are present for $r_{0}<1$ are studied in [25], although not from this bifurcation point-of-view.

\section{INSERT FIGURES 1 and 2 NEAR HERE}

We now consider an evolutionary version of equation (23) to which we can apply the results of Sections 4 and 5. For our application we consider the case when the inherent (density and predation free) per capita birth rate $b$ and the predation protection factor $s$ are subject to evolutionary adaptation. We think of these per capita quantities as characteristics of an individual and that they are determined by a suite of phenotypic traits $\hat{v}$ of the individual. Thus, $b=b(\hat{v})$ and $s=s(\hat{v})$. We assume that there is a trait vector that maximizes $b$ and one that maximizes $s$, but these optimizing trait vectors are not the same. The idea is that there are trade-offs in the allocation of energy, behavioral activities, and resources towards reproduction and towards the avoidance of 
predators. For example, traits that promote physiological and behavioral characteristics promote successful herding or flocking or schooling in order to avoid predation are not necessarily traits that make for optimal reproduction.

Since we have set no units or scales for the traits, we assume without loss in generality that $b$ is maximal at $\hat{v}=\hat{0}$ and $s$ is maximal at $\hat{v}=(1,1, \cdots, 1)^{T}$. Specifically, we assume (as is often done in evolutionary models [28]) that these coefficients have a multi-variate Gaussian-type distribution about these maximal points:

$$
b(\hat{v})=\beta \exp \left(-\sum_{i=1}^{n} \frac{v_{i}^{2}}{2 b_{i}}\right), \quad s(\hat{v})=s_{0} \exp \left(-\sum_{i=1}^{n} \frac{\left(v_{i}-1\right)^{2}}{2 s_{i}}\right)
$$

where $b_{i}$ and $s_{i}$ are positive real numbers (variances), $\beta>0$ is the maximal possible value of $b(\hat{v})$, and $s_{0} \geq 0$ is the maximal possible value of $s(\hat{v})$. The resulting $1 \times 1$ projection matrix $P(x, \hat{u}, \hat{v})$ for (23) is independent of $\hat{u}$ and its single entry $p_{11}(x, \hat{v})$ equals the dominant eigenvalue, i.e. $p_{11}(x, \hat{v})=r(x, \hat{v})$ where

$$
r(x, \hat{v})=\beta e^{-\alpha} \exp \left(-\sum_{i=1}^{n} \frac{v_{i}^{2}}{2 b_{i}}\right) \exp \left(-c x+\alpha \frac{s(\hat{v}) x}{1+s(\hat{v}) x}\right) .
$$

The Darwinian equations (6) are

$$
\begin{aligned}
x(t+1) & =r(x(t), \hat{u}(t)) x(t) \\
\hat{u}(t+1) & =\hat{u}(t)+M \nabla_{\hat{v}} \ln r(x(t), \hat{u}(t))
\end{aligned}
$$

with

$$
\nabla_{\hat{v}} \ln r(x, \hat{v})=-\left(\begin{array}{c}
\frac{v_{1}}{b_{1}} \\
\vdots \\
\frac{v_{n}}{b_{n}}
\end{array}\right)-\alpha \frac{s(\hat{v}) x}{(1+s(\hat{v}) x)^{2}}\left(\begin{array}{c}
\frac{v_{1}-1}{s_{1}} \\
\vdots \\
\frac{v_{n}-1}{s_{n}}
\end{array}\right) .
$$

Since

$$
\left.\nabla_{\hat{v}} \ln r(0, \hat{v})\right|_{\hat{v}=\hat{u}}=-\left(\begin{array}{c}
\frac{u_{1}}{b_{1}} \\
\vdots \\
\frac{u_{n}}{b_{n}}
\end{array}\right)
$$

we see that the only critical trait is

$$
\hat{u}^{*}=\hat{0} \text {. }
$$

and hence the only extinction equilibrium is $(x, \hat{u})=(0, \hat{0})$. Our bifurcation parameter $r_{0}^{*}=r(0, \hat{0})$ reduces to

$$
r_{0}^{*}=\beta e^{-\alpha} .
$$

Under the added assumption that the traits are not correlated, so that the variance-covariance matrix $M=\operatorname{diag}\left(\sigma_{i}^{2}\right)$ is a diagonal matrix, the model equations for our evolutionary version of (23) are

$$
\begin{aligned}
x(t+1) & =r_{0}^{*} \exp \left(-\sum_{i=1}^{n} \frac{u_{i}^{2}(t)}{2 b_{i}}\right) x(t) \exp \left(-c x(t)+\alpha \frac{s(\hat{u}(t)) x(t)}{1+s(\hat{u}(t)) x(t)}\right) \\
u_{i}(t+1) & =u_{i}(t)-\sigma_{i}^{2}\left(\frac{u_{i}(t)}{b_{i}}+\alpha \frac{u_{i}(t)-1}{s_{i}} \frac{s(\hat{u}(t)) x(t)}{(1+s(\hat{u}(t)) x(t))^{2}}\right)
\end{aligned}
$$


for $i=1, \cdots, n$. Our goal is to apply Corollary 4 to these difference equations, toward which end we must consider $\mathrm{H} 2$ and $\mathrm{H} 5$.

H2 holds with $\tilde{p}_{11}(\hat{v})=r_{0}(\hat{v})$ and $\bar{p}(x, \hat{u}, \hat{v})=\bar{r}(x, \hat{v})$, since $\bar{r}(0, \hat{v}) \equiv 1$. A calculation shows that the Hessian $H\left(0, \hat{u}^{*}, \hat{u}^{*}\right)=\left[\partial_{v_{i} v_{j}}^{0} \ln \bar{r}\right]$ is the diagonal matrix

$$
H\left(0, \hat{u}^{*}, \hat{u}^{*}\right)=\operatorname{diag}\left[-\frac{1}{b_{i}}\right]
$$

Thus, H5 holds (see Remark 4). By Theorem 3 the bifurcating continuum of positive equilibrium pairs $\left(r_{0}^{*},(x, \hat{u})\right)$, near the bifurcation point $(1,(0, \hat{0}))$, has the parametric representation

$$
\begin{aligned}
r_{0}^{*}(\varepsilon) & =1+\kappa^{*} \varepsilon+O\left(\varepsilon^{2}\right) \\
x(\varepsilon) & =\varepsilon+O\left(\varepsilon^{2}\right) \\
\hat{u}(\varepsilon) & =\hat{u}_{1} \varepsilon+O\left(\varepsilon^{2}\right)
\end{aligned}
$$

for $\varepsilon \gtrsim 0$ where, by the formulas (18) and (19),

$$
\begin{gathered}
\kappa^{*}=c-\alpha s_{0} \exp \left(-\sum_{i=1}^{n} \frac{1}{2 s_{i}}\right) \\
\hat{u}_{1}=\alpha s_{0} \exp \left(-\sum_{i=1}^{n} \frac{1}{2 s_{i}}\right)\left(\begin{array}{c}
\frac{b_{1}}{s_{1}} \\
\vdots \\
\frac{b_{n}}{s_{n}}
\end{array}\right) \in \mathbb{R}_{+}^{n} .
\end{gathered}
$$

By Corollary $4(\mathrm{a}, \mathrm{b})$, the direction of bifurcation determines the stability of the bifurcating positive equilibria provided

$$
\sigma_{i}^{2}<2 b_{i} \text { for all } i=1, \cdots, n
$$

that is to say, provided the speed of evolution is not too fast. Under this assumption, we have the following conclusions concerning the bifurcation at $r_{0}^{*}=1$ for the Darwinian model (24).

1. (Forward bifurcations) The bifurcation of the continuum $\mathcal{C}$ of positive equilibria for (24b) is forward and consequently stable if

$$
\alpha s_{0} \exp \left(-\sum_{i=1}^{n} \frac{1}{2 s_{i}}\right)<c .
$$

This occurs if the negative density effects, as described by the Ricker coefficient $c$, are large enough to dominate the positive effects from the Allee effect attributed to density protection from predation, as encapsulated by the quantity on the left side of the inequality (27). Thus, mechanisms that promote a forward bifurcation are: a low predation intensity $\alpha$, a low maximum possible predation protection coefficient $s_{0}$, and small variances $s_{i}$ (i.e. the largest predator protection coefficients $s(\hat{v})$ are attained only for trait vectors narrowly distributed around the maximal trait vector $\hat{v}=$ $\left.(1, \cdots, 1)^{T}\right)$.

Note that the entries in $\hat{u}_{1}$ in $(25 \mathrm{c})$ are positive if $\alpha s_{0}>0$, i.e. if both predation and predation protection are present. In this case, we see that near the bifurcation point, the trait components $u_{i}(\varepsilon)$ of the bifurcating positive equilibria are positive. As a result, for $r_{0}^{*} \gtrsim 1$ the stable, positive 
equilibria have trait components that do not maximize the inherent birth rate. Indeed, an even stronger conclusion follows directly from the trait equilibrium equations (24b):

$$
\frac{u_{i}}{b_{i}}+\alpha \frac{u_{i}-1}{s_{i}} \frac{s(\hat{u}) x}{(1+s(\hat{u}) x)^{2}}=0 \text { for all } i=1, \ldots, n .
$$

This shows, when $\alpha>0$ and $s_{0}>0$, that for any positive equilibrium $(x, \hat{u})$ of $(24)$, the equilibrium trait components $u_{i}$ cannot equal 0 or 1 . For those equilibrium pairs from the continuum $\mathcal{C}$ the trait components form a continuum of equilibrium trait vectors $\hat{u}$ which must, therefore, have components that lie entirely in the interval $0<u_{i}<1$ (whether the equilibria are stable or not). It follows that for those positive equilibria from $\mathcal{C}$ which are in fact stable (such as those for $r_{0}^{*} \gtrsim 1$ ), we can say that evolution selects a vector of traits that neither maximizes the inherent birth rate $b(\hat{u})$ (which occurs at $\hat{u}=\hat{0}$ ) nor the predator protection coefficient $s(\hat{u})$ (which occurs at $\hat{u}=(1,1, \cdots, 1)^{T}$ ). One might say, then, that evolution trades-off a smaller inherent birth rate in favor of some predator protection.

When predation and/or predation protection is absent $\left(\alpha=0\right.$ and/or $\left.s_{0}=0\right)$ in the model, then clearly inequality (27) holds and the bifurcation at $r_{0}^{*}=1$ is forward and stable. In this case, the equilibrium equation (28) for the traits $u_{i}$ implies $u_{i}=0$ for any positive equilibrium pair and, not surprisingly, evolution selects to maximize the inherent bifurcation rate $b(\hat{u})$.

(2) (Backward bifurcations). The bifurcation of the continuum $\mathcal{C}$ of positive equilibria for (24b) is backward and consequently unstable if

$$
\alpha s_{0} \exp \left(-\sum_{i=1}^{n} \frac{1}{2 s_{i}}\right)>c .
$$

This occurs only if predation is present $\alpha>0$ and density protection from predation is also present $s_{0}>0$. Inequality (29) holds if predation intensity $\alpha$ and/or predator protection $s_{0}$ are large (relative to the negative density effects $c$ ). Also promoting a backward bifurcation are large variances $s_{i}$, that is to say, when a high level of predator protection $s(\hat{v})$ is attained for a wide distribution of trait vectors $\hat{v}$.

Our general results in Section 5 concern equilibrium properties in a neighborhood of the bifurcation point and do not imply anything about the dynamics outside such a neighborhood. As in the non-evolutionary model (23), we expect it to be true that the positive equilibria on the continuum $\mathcal{C}$ for the evolutionary model (24) do not necessarily retain the stability properties that they possess near the bifurcation point. In particular, in the case of a forward/stable bifurcation we would expect that, at least for some model parameter values, the stable positive equilibria will destabilize with increasing $r_{0}^{*}$ and even give rise to a sequence of bifurcations that result complicated, chaotic dynamics. In the case of a backward/unstable bifurcation, in addition to this phenomenon, we would also anticipate the potential for strong Allee effects on an interval of $r_{0}^{*}$ values less than 1 . We will not study these questions about the dynamics of (24) in this paper where our theory is focussed on the local bifurcation at $r_{0}^{*}=1$.

However, we can provide a few selected numerical simulations that, in addition to illustrating the local bifurcation predicted by our theorems, also illustrate the kinds of secondary bifurcations and strong Allee effects since in the non-evolutionary case (cf. Figure 2). Figure 3 shows two sample bifurcation diagrams for the evolutionary model (24) with two traits, i.e. $n=2$. The plots in Figure $3 \mathrm{~A}$ are from parameter values for which $\kappa^{*}>0$ and, hence, a forward, stable bifurcation occurs at 
$r_{0}^{*}=1$. As with the non-evolutionary version of the model in Figure 1, further increases in $r_{0}^{*}$ result in the familiar period doubling route to chaotic dynamics. In Figure 3B the same parameter values are used except that the predator protection coefficient $s_{0}$ is increased to the extent that $\kappa^{*}<0$ and, as a result, a backward, unstable bifurcation occurs. The result is a bifurcation diagram that shows a saddle-node bifurcation (at $r_{0}^{*} \approx 0.4$ ) creating an interval of strong Allee effects with both a stable extinction equilibrium $(x, \hat{u})=(0, \hat{0})$ and a stable positive equilibrium. In this example, one sees from Figure 3B that the positive equilibrium loses stability through a period doubling at a value of $r_{0}^{*}$ less than 1 . This results in an interval of $r_{0}^{*}$ values less than 1 for which there is a strong Allee effect that involves a stable positive 2-cycle instead of a positive equilibrium. (The oscillations in the traits $u_{i}$ are small amplitude in Figure 3B.)

\section{INSERT FIGURE 3 NEAR HERE}

\section{Concluding Remarks}

A fundamental property of population dynamic models, when the extinction state destabilizes due to a change in a model parameter, is the occurrence of a bifurcation which results in the presence of positive equilibria. Typically the stability of these bifurcating equilibria depend on the direction of bifurcation (Theorem 1). In this paper we investigate this basic bifurcation phenomenon for an evolutionary version of a general matrix model for the dynamics of a structured population. The model assumes that the entries of the model's projection matrix (i.e. the per capita birth, survival and class transition rates) depend on a vector of phenotypic traits, each of which is subject to Darwinian evolution, and tracks the dynamics of the population and the vector of mean traits [28]. We define the notion of a critical trait vector, which is associated with the existence of an extinction equilibrium in the model, and obtain conditions under which an extinction equilibrium destabilizes (Theorem 2) and conditions under which a continuum of positive equilibria bifurcates from the extinction equilibrium, as the inherent population growth rate (at the critical trait) increases through 1 (Theorem 3). We further obtain conditions under which stability of the bifurcating equilibria is determined by the direction of bifurcation and conditions under which it is not (Theorem 3).

It is shown in [23] that the bifurcating continuum $\mathcal{C}^{*}$ of positive equilibria in Theorem 3 has a global extent in $\mathbb{R}_{+} \times\left(\mathbb{R}_{+}^{m} \times V\right)$ in that it connects to the boundary of this cone ( $\infty$ is included in the boundary). In general, however, the stability/instability results in Theorem 3 hold only in a neighborhood of the bifurcation point. This is illustrated in the example studied in Section 6 where secondary bifurcations occur outside the neighborhood of the bifurcation point. Whether or not such bifurcations occur are model dependent (which is true in non-evolutionary matrix models as well).

In non-evolutionary matrix models, backward bifurcations are often associated with strong Allee effects, i.e. multiple attractors for values of $r_{0}^{*}<1$ one of which is extinction and the other which is a survival attractor [10]. While conditions sufficient for the occurrence of a backward bifurcation are given in Section 5, its relation to strong Allee effects is not investigated in this paper. A backward induced strong Allee effect is shown to occur, by simulations, in the example studied in Section 6 . 


\section{Acknowledgements}

J. M. Cushing and A. Veprauskas were supported by the U.S. National Science Foundation grant DMS 0917435.

A. A. Pinto thanks the support of LIAAD - INESC TEC through program PEst, the Faculty of Sciences of University of Porto and Portuguese Foundation for Science and Technology (FCT - Fundação para a Ciência e a Tecnologia) through the Project "Dynamics, Optimization and Modelling, with with reference PTDC/MAT-NAN/6890/2014. This work is financed by the ERDF - European Regional Development Fund through the Operational Programme for Competitiveness and Internationalisation - COMPETE 2020 Programme, and by National Funds through the FCT - Fundação para a Ciência e a Tecnologia (Portuguese Foundation for Science and Technology) within project «POCI-01-0145-FEDER-006961.

F. Martins thanks the financial support of Portuguese Foundation for Science and Technology (FCT - Fundação para a Ciência e a Tecnologia) through a PhD. scholarship of the programme MAP-PDMA. (Reference: PD/BD/105726/2014).

The authors are grateful for the comments of two anonymous reviewers and the handling editor, which were exceptionally helpful in improving the paper.

\section{Appendix}

Lemma 1 Assume H2 and H5 hold. Then $\hat{w}_{L}^{T}\left[\nabla_{\hat{v}}^{0} q_{i j}^{T} \hat{u}_{1}\right] \hat{w}_{R}=0$.

Proof. Consider the equality

$$
P(\hat{0}, \hat{u}, \hat{u}) \hat{w}_{R}(\hat{0}, \hat{u})=r(\hat{0}, \hat{u}, \hat{u}) \hat{w}_{R}(\hat{0}, \hat{u}) .
$$

which holds by the definition of $r(\hat{0}, \hat{u}, \hat{u})$ as an eigenvalue with a positive right eigenvector $\hat{w}_{R}(\hat{0}, \hat{u})$. Let $\hat{p}_{i}=\hat{p}_{i}(\hat{0}, \hat{u}, \hat{u})$ denote the $i$-th column of $P=P(\hat{0}, \hat{u}, \hat{u})$. We want to take the Jacobian of both sides of equation (30) with respect to $\hat{u}$. To do this we let $J_{\hat{y}}[\hat{\omega}(\hat{y})]$ denote the Jacobian of a vector valued function $\hat{\omega}(\hat{y})$ of a vector $\hat{y}$.

The right side of $(30)$ is a vector valued function of the form $\tau(\hat{y}) \hat{\omega}(\hat{y})$ for a scalar valued function $\tau(\hat{y})$. Applying the general formula

$$
J_{\hat{y}}[\tau(\hat{y}) \hat{\omega}(\hat{y})]=\hat{\omega}(\hat{y}) \nabla_{\hat{y}} \tau(\hat{y})^{T}+\tau(\hat{y}) J_{\hat{y}}[\hat{\omega}(\hat{y})]
$$

and recalling (8) in Remark 2, we find that the Jacobian of the right side of (30) with respect to $\hat{u}$ is

$$
\hat{w}_{R}(\hat{0}, \hat{u})\left(\nabla_{\hat{u}} r^{T}+\nabla_{\hat{v}} r^{T}\right)+r J_{\hat{u}}\left[\hat{w}_{R}(\hat{0}, \hat{u})\right]=\hat{w}_{R}(\hat{0}, \hat{u}) \nabla_{\hat{v}} r^{T}+r J_{\hat{u}}\left[\hat{w}_{R}(\hat{0}, \hat{u})\right] .
$$

To calculate the Jacobian of the left-hand side of (30), we write

$$
P \hat{w}_{R}(\hat{0}, \hat{u})=\sum_{i=1}^{m} w_{i}^{R}(\hat{0}, \hat{u}) \hat{p}_{i}
$$

where $w_{i}^{R}(\hat{0}, \hat{u})$ are the components of the vector $\hat{w}_{R}(\hat{0}, \hat{u})$ and apply the product rule (31) to each term. Noting (7) in Remark 2 we get

$$
P J_{\hat{u}}\left[\hat{w}_{R}(\hat{0}, \hat{u})\right]+\sum_{i=1}^{m} w_{i}^{R}(\hat{0}, \hat{u}) J_{\hat{v}}\left[\hat{p}_{i}\right] .
$$


Equating the Jacobians of the left and right sides of (30) we have

$$
P J_{\hat{u}}\left[\hat{w}_{R}(\hat{0}, \hat{u})\right]+\sum_{i=1}^{m} w_{i}^{R}(\hat{0}, \hat{u}) J_{\hat{v}}\left[\hat{p}_{i}\right]=\hat{w}_{R}(\hat{0}, \hat{u}) \nabla_{\hat{v}} r^{T}+r J_{\hat{u}}\left[\hat{w}_{R}(\hat{0}, \hat{u})\right]
$$

or

$$
\left(P-r I_{m}\right) J_{\hat{u}}\left[\hat{w}_{R}(\hat{0}, \hat{u})\right]=\hat{w}_{R}(\hat{0}, \hat{u}) \nabla_{\hat{v}} r^{T}-\sum_{i=1}^{m} w_{i}^{R}(\hat{0}, \hat{u}) J_{\hat{v}}\left[\hat{p}_{i}\right]
$$

which in turn can be rewritten as the $m$ equations

$$
\left(P-r I_{m}\right) \partial_{u_{i}}\left(\hat{w}_{R}(\hat{0}, \hat{u})\right)=\left(\partial_{v_{i}} r I_{m}-\partial_{v_{i}} P\right) \hat{w}_{R}(\hat{0}, \hat{u}) \text { for } 1 \leq i \leq n .
$$

The matrix $P-r I_{m}$ is singular and by the Fredholm alternative, the solubility of these equations imply the $m$ orthogonality conditions

$$
\hat{w}_{L}^{T}(\hat{0}, \hat{u})\left(\partial_{v_{i}} r I_{m}-\partial_{v_{i}} P\right) \hat{w}_{R}(\hat{0}, \hat{u})=0
$$

are satisfied. Solving for $\partial_{v_{i}} r$ and recalling that the eigenvectors are normalized so that $\hat{w}^{L}(\hat{0}, \hat{u})^{T} \hat{w}_{R}(\hat{0}, \hat{u})=$ 1 , we find

$$
\partial_{v_{i}} r=\hat{w}_{L}^{T}(\hat{0}, \hat{u}) \partial_{v_{i}} P \hat{w}_{R}(\hat{0}, \hat{u}) \text { for } 1 \leq i \leq n .
$$

Since $\partial_{v_{i}}^{0} r=0$ by definition of a critical trait vector $\hat{u}^{*}$, when setting $\hat{u}=\hat{u}^{*}$ and $r_{0}^{*}=1$ in these expressions we get

$$
\hat{w}_{L}^{T} \partial_{v_{k}}^{0} Q \hat{w}_{R}=0 \text { for } 1 \leq k \leq n .
$$

Let $u_{1, k}$ denote the scalar components of the vector $\hat{u}_{1}$. Then

$$
\nabla_{\hat{v}}^{0} q_{i j}^{T} \hat{u}_{1}=\sum_{k=1}^{n} u_{1, k} \partial_{v_{k}}^{0} q_{i j}
$$

and

$$
\left[\nabla_{\hat{v}}^{0} q_{i j}^{T} \hat{u}_{1}\right]=\sum_{k=1}^{n} u_{1, k}\left[\partial_{v_{k}}^{0} q_{i j}\right]=\sum_{k=1}^{n} u_{1, k} \partial_{v_{k}}^{0} Q
$$

From

$$
\hat{w}_{L}^{T}\left[\nabla_{\hat{v}}^{0} q_{i j} \hat{u}_{1}\right] \hat{w}_{R}=\sum_{k} u_{1, k}\left(\hat{w}^{L} \partial_{v_{k}}^{0} Q \hat{w}_{R}\right)
$$

and (33) it follows that $\hat{w}_{L}^{T}\left[\nabla_{\hat{v}}^{0} q_{i j} \hat{u}_{1}\right] \hat{w}_{R}=0$. 


\section{References}

[1] P. A. Abrams, Modelling the adaptive dynamics of traits involved in inter- and intraspecific interactions: An assessment of three methods, Ecology Letters, 4 (2001), pp. 166-175.

[2] P. A. Abrams, 'Adaptive Dynamics' vs. 'adaptive dynamics', Journal of Evolutionary Biology, 18 (2005), pp. 1162-1165.

[3] A. Bernman and R. J. Plemmons, Nonnegative Matrices in the Mathematical Sciences, Classics in Applied Mathematics. SIAM, Philadelphia, 1994.

[4] H. Caswell, Matrix Population Models: Construction, Analysis and Interpretation, Second Edition, Sinauer Associates, Inc. Publishers, Sunderland, Massachusetts, 2001.

[5] F. Courchamp, L. Berec and J. Gascoigne, Allee Effects in Ecology and Conservation, Oxford University Press, Oxford, Great Britain, 2008.

[6] J. M. Cushing, A bifurcation theorem for Darwinian matrix models, Nonlinear Studies, 17 (2010), pp. 1-13.

[7] J. M. Cushing, An Introduction to Structured Population Dynamics, CBMS-NSF Regional Conference Series in Applied Mathematics, Vol. 71, SIAM, Philadelphia, 1998.

[8] J. M. Cushing, Matrix Models and Population Dynamics, a chapter in Mathematical Biology (Mark Lewis, A. J. Chaplain, James P. Keener, Philip K. Maini eds.), IAS/Park City Mathematics Series Vol. 14, American Mathematical Society, Providence, I, 2009, pp. 47-150.

[9] J. M. Cushing, On the relationship between $r$ and $R_{0}$ and its role in the bifurcation of equilibria of Darwinian matrix models, Journal of Biological Dynamics, 5 (2011), pp. 277-297.

[10] J. M. Cushing, Backward bifurcations and strong Allee effects in matrix models for the dynamics of structured populations, Journal of Biological Dynamics, 8 (2014), pp. 57-73.

[11] J. M. Cushing, One dimensional maps as population and evolutionary dynamic models, Applied Analysis in Biological and Physical Sciences, Springer Proceedings in Mathematics \& Statistics, Volume 186, (J. M. Cushing, M. Saleem, H. M. Srivastava, M. A. Khan, M. Merajuddin, eds.), Springer, India, 2016.

[12] B. Dennis, Allee effects: Population growth, critical density, and the chance of extinction, Natural Resource Modeling 3 (1989), 481-538.

[13] F. Dercole and S. Rinaldi, Analysis of Evolutionary Processes: The Adaptive Dynamics Approach and Its Applications, Princeton University Press, New Jersey, 2008

[14] S. N. Elaydi, An Introduction to Difference Equations, Springer-Verlag, New York, 1996.

[15] H. Keilhöfer, Bifurcation Theory: An Introduction with Applications to PDEs, Applied Mathematical Sciences 156, Springer, New York, 2004.

[16] R. Lande, Natural selection and random genetic drift in phenotypic evolution, Evolution, 30 (1976), pp. 314-334. 
[17] R. Lande, A quantitative genetic theory of life history evolution, Ecology, 63 (1982), pp. 607615.

[18] L. P. Lefkovitch, The study of population growth in organisms grouped by stage, Biometrics, 21 (1965), 1-18

[19] P. H. Leslie, On the use of matrices in certain population mathematics, Biometrika, 33 (1945), pp. 183-212.

[20] P. H. Leslie, Some further notes on the use of matrices in population mathematics, Biometrika, 35 (1948), 213-245.

[21] E. G. Lewis, On the generation and growth of a population, Sankhya, 6 (1942), 93-96.

[22] B. J. McGill and J. S. Brown, Evolutionary game theory and adaptive dynamics of continuous traits, Annual Review of Ecology, Evolution, and Systematics, 38 (2007), pp. 403-435.

[23] E. P. Meissen, K. R. Salau, J. M. Cushing, A global bifurcation theorem for Darwinian matrix models, to appear in the Journal of Difference Equations and Applications

[24] D. A. Roff, The Evolution of Life Histories: Theory and Analysis, Chapman and Hall, New York, 1992.

[25] S. Schreiber, Allee effects, extinctions, and chaotic transients in simplie population models, Theoretical Population Biology 64 (2003), 201-209.

[26] M. B. Usher, A matrix approach to the management of renewable resources, with special reference to selection forests, Journal of Applied Ecology 3 (1966), 355-367.

[27] A. Veprauskas and J. M. Cushing, Evolutionary dynamics of a multi-trait semelparous model, Discrete and Continuous Dynamical Systems Series B, 21, No. 2 (2016), 655-676.

[28] T. L. Vincent and J. S. Brown, Evolutionary Game theory, Natural selection, and Darwinian dynamics, Cambridge University Press, 2005. 




Figure 1. Shown is a generic plot of the bifurcating continuum $C$ for equation (23) when $\alpha s>c$ and, consequently, a backward (unstable) bifurcation occurs at the point $\left(r_{0}, x\right)=(1,0)$. The question mark indicates that although the positive equilibria on the upper branch $x_{2}\left(r_{0}\right)$ are (locally asymptotically) stable near the saddle-node bifurcation point $\left(r_{c r}, x_{c r}\right)$, they can, depending on model parameter values, destabilize further along the continuum $C$.
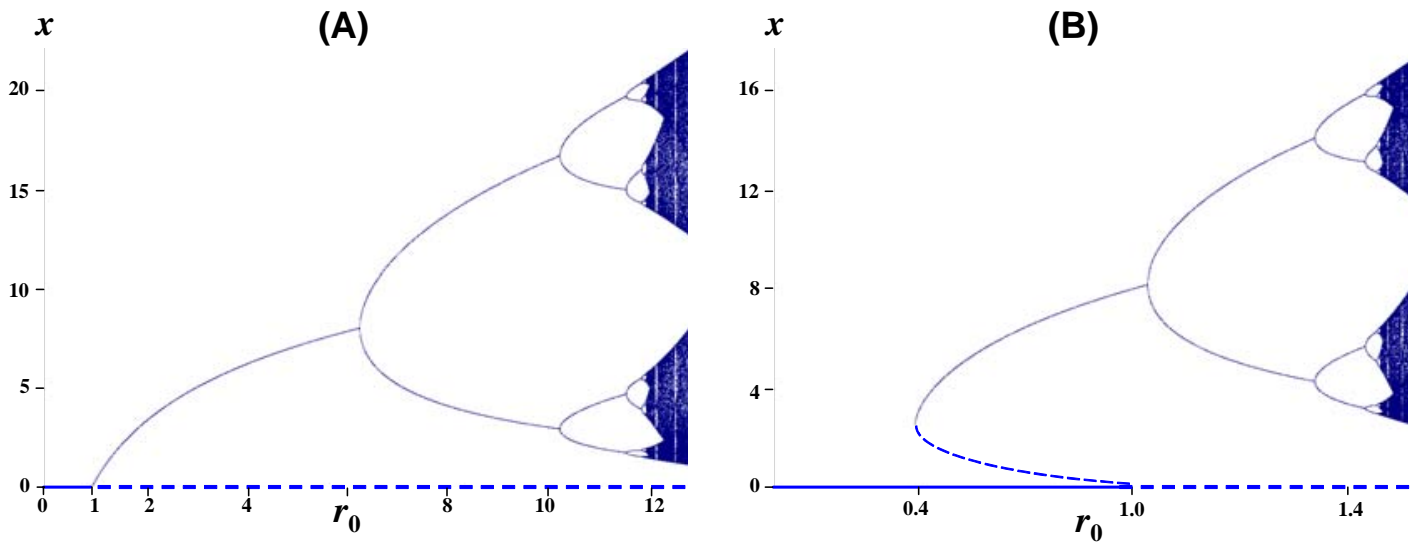

FiguRE 2. Sample bifurcation diagrams for equation (23) with $c=0.3$ and $\alpha=3$.

A. $s=0.05$ and $\kappa=c-\alpha s=0.15>0$ so that the bifurcation at $r_{0}=\beta e^{-3}=1$ is forward and stable.

B. $s=1$ and $\kappa=-2.7<0$ so that the bifurcation at $r_{0}=\beta e^{-3}=1$ is backward and unstable (dashed line). 

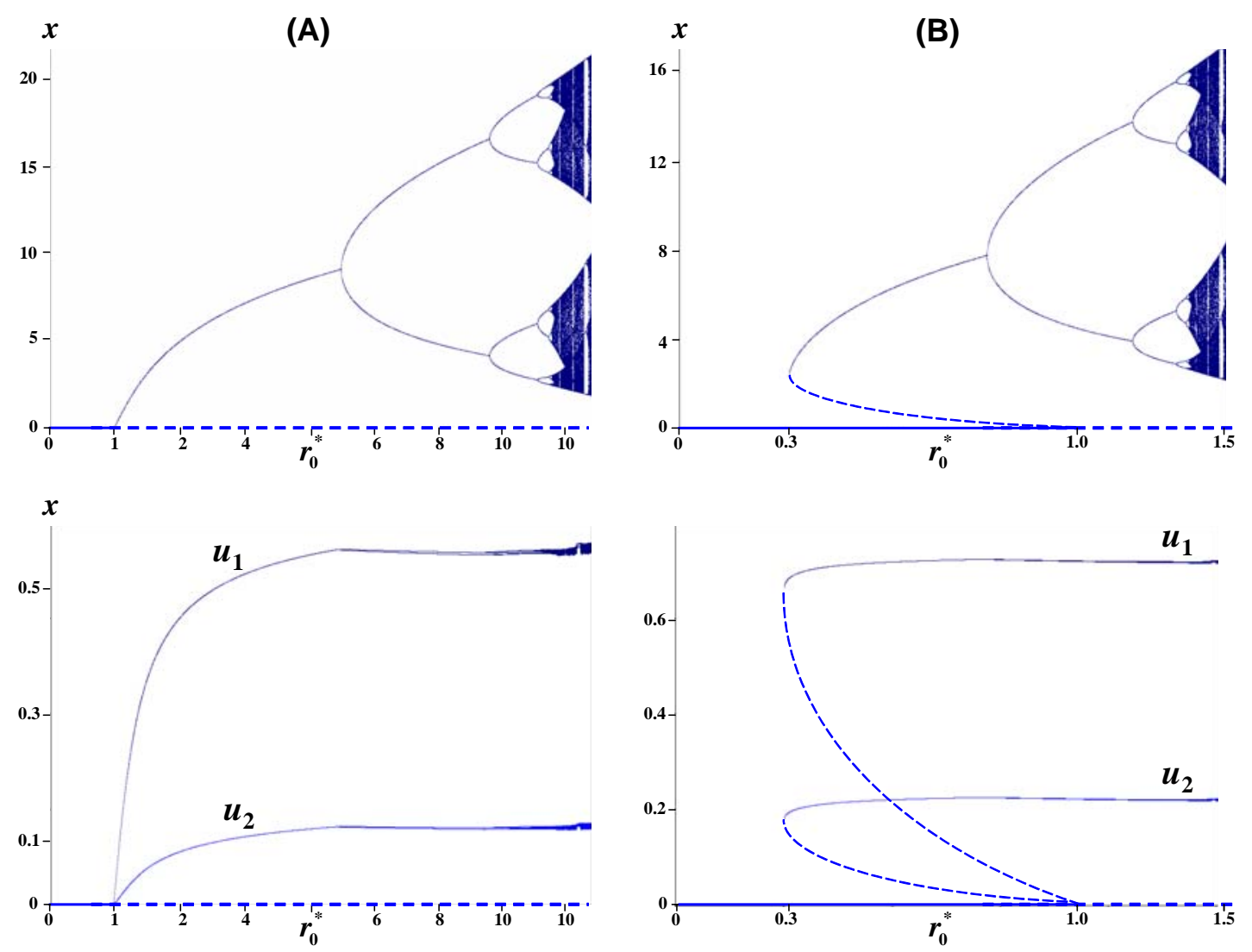

Figure 3. Sample bifurcation diagrams for equations (24) with $c=0.3, \alpha=3, b_{1}=$ $3, b_{2}=1, s_{1}=1$, and $s_{2}=3$.

A. $s_{0}=0.1$ and $\kappa^{*}=3\left(1-e^{-2 / 3}\right) / 10 \approx 0.146>0$ so that the bifurcation at $r_{0}^{*}=\beta e^{-3}=1$ is forward and stable.

B. $s=1$ and $\kappa^{*}=3\left(1-10 e^{-2 / 3}\right) / 10 \approx-1.2403<0$ so that the bifurcation at $r_{0}^{*}=\beta e^{-3}=1$ is backward and unstable (dashed lines). 
(A)
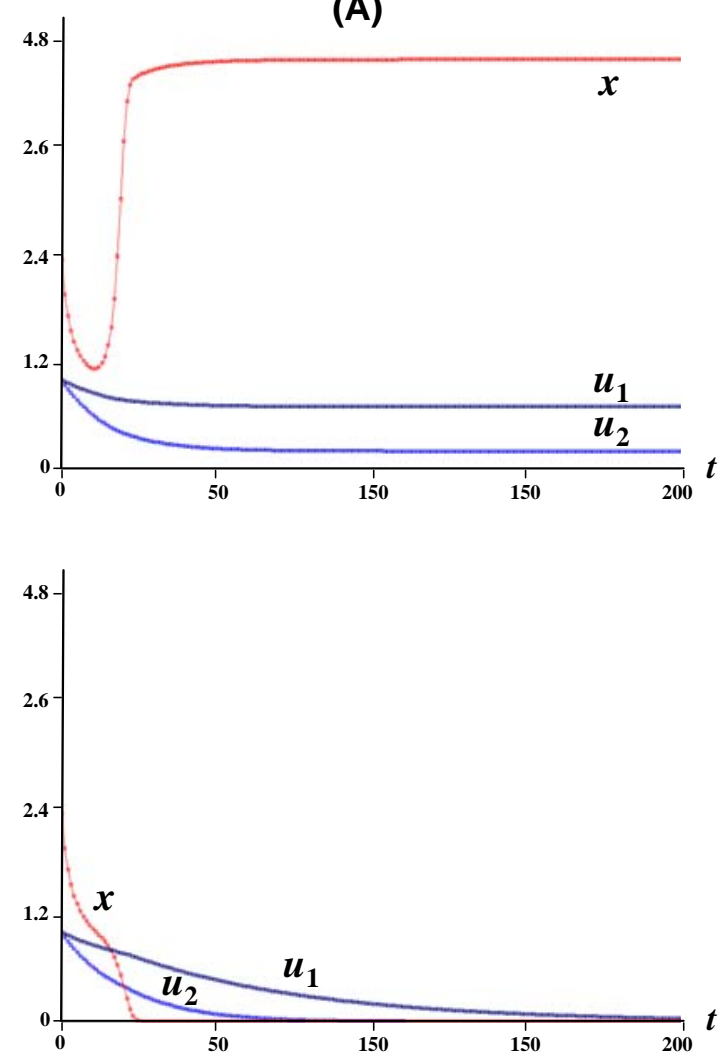

(B)
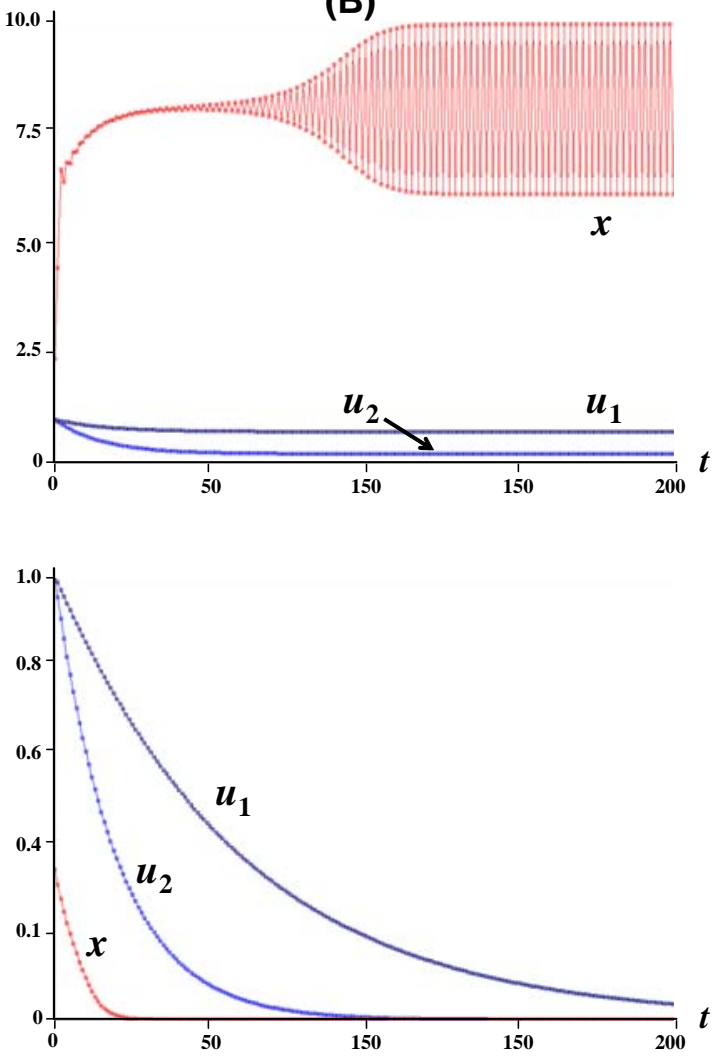

FIGURE 4. Shown are sample orbits for equations (24) with the same parameter values used in Figure 3B when a backward bifurcation creates an interval of $r_{0}^{*}<1$ for which there is a strong Allee effect.

A. For $r_{0}^{*}=\beta e^{-3}=0.4$ the bifurcation diagram Figure 3B shows a stable extinction equilibrium and a stable positive equilibrium. The upper graph in column $\mathrm{A}$ shows plots of the solution with initial conditions $x=2.35, u_{1}=u_{2}=1$ and that tends to the positive equilibrium. The lower graph shows plots of the solution with initial conditions $x=2.33, u_{1}=u_{2}=1$ and that tends to the extinction equilibrium $(x, \hat{u})=(0, \hat{0})$.

B. For $r_{0}^{*}=\beta e^{-3}=0.9$ the bifurcation diagram Figure 3B shows a stable extinction equilibrium and a stable 2-cycle. The upper graph in column B shows plots are of the solution with initial conditions $x=2.35, u_{1}=u_{2}=1$ and that tends to the positive 2 -cycle. The lower graph in column B shows plots of the solution with initial conditions $x=0.35, u_{1}=u_{2}=1$ and that tends to the extinction equilibrium $(x, \hat{u})=(0, \hat{0})$. 


\title{
A Bifurcation Theorem for Evolutionary Matrix Models with Multiple Traits
}

\author{
J. M. Cushing ${ }^{\dagger, 1,2}$, F. Martins ${ }^{3}$, A. A. Pinto ${ }^{3}$, Amy Veprauskas ${ }^{2}$ \\ ${ }^{1}$ Department of Mathematics \\ ${ }^{2}$ Interdisciplinary program in Applied Mathematics \\ University of Arizona \\ 617 N Santa Rita \\ Tucson, AZ 85721 \\ ${ }^{3}$ Department of Mathematics \\ Faculty of Sciences \\ University of Porto and LIAAD-INESC \\ Rua do Campo Alegre 687 \\ 4169-007 Porto, Portugal
}

\begin{abstract}
One fundamental question in biology is population extinction and persistence, i.e., stability/instability of the extinction equilibrium and of non-extinction equilibria. In the case of nonlinear matrix models for structured populations, a bifurcation theorem answers this question when the projection matrix is primitive by showing the existence of a continuum of positive equilibria that bifurcates from the extinction equilibrium as the inherent population growth rate passes through 1 . This theorem also characterizes the stability properties of the bifurcating equilibria by relating them to the direction of bifurcation, which is forward (backward) if, near the bifurcation point, the positive equilibria exist for inherent growth rates greater (less) than 1. In this paper we consider an evolutionary game theoretic version of a general nonlinear matrix model that includes the dynamics of a vector of mean phenotypic traits subject to natural selection. We extend the fundamental bifurcation theorem to this evolutionary model. We apply the results to an evolutionary version of a Ricker model with an added Allee component. This application illustrates the theoretical results and, in addition, several other interesting dynamic phenomena, such as backward bifurcation induced strong Allee effects and survival when multiple traits evolve, but extinction if only one (or no) trait evolves.
\end{abstract}

Key words: Nonlinear matrix models, structured population dynamics, evolutionary game theory, bifurcation, equilibria, stability

AMS subject classifications: 92D25, 92D15, 39A28, 37G99

\footnotetext{
${ }^{\dagger}$ Corresponding author: cushing@math.arizona.edu
} 


\section{Introduction}

We consider a discrete time model

$$
\hat{x}(t+1)=P(\hat{x}(t)) \hat{x}(t), \quad t \in \mathbb{N}_{0}=\{0,1,2, \ldots\}
$$

for the dynamics of a biological population whose individuals are classified into a finite number of discrete classes. Here $\hat{x}: \mathbb{N}_{0} \rightarrow \overline{\mathbb{R}}_{+}^{m}$ is a sequence of $m$-dimensional column vectors consisting of class specific population densities, where $\overline{\mathbb{R}}_{+}^{m}$ is the closure of the positive cone $\mathbb{R}_{+}^{m}$ in $m$-dimensional Euclidean space $\mathbb{R}^{m}$. Recursive formulas (1), called matrix models, are widely utilized to describe the dynamics of populations in which individuals are classified according to age, size, life cycle stage, spatial location, genetic composition, etc., indeed virtually any classification scheme of interest $[4,7]$.

The entries $p_{i j}(\hat{x})$ of the projection matrix $P(\hat{x})$ are chosen by a modeler to describe classspecific, per capita (individual) birth and survival rates and to account for transitions of individuals from one class to another. As indicated, these entries can be dependent on the densities in the demographic vector $\hat{x}$, dependencies that make the dynamic model nonlinear. Classic examples of matrix models for structured population dynamics include the age, size, and stage structured models of Leslie and Lewis [19, 20, 21], Usher [26], and Lefkovitch [18].

Of fundamental importance to a biological population is its avoidance of extinction. We refer to the equilibrium $\hat{x}=\hat{0}$ solution of (1) as the extinction equilibrium. If the extinction equilibrium is an attractor, then the population is threatened with extinction. This leads to the study of the stability properties (local and global) of the extinction equilibrium. The linearization principle [14] leads one to consider the eigenvalues of the Jacobian obtained from (1) evaluated at the extinction equilibrium, which is the inherent projection matrix $P(\hat{0})$ (inherent means density free). If all eigenvalues of $P(\hat{0})$ lie in the complex unit circle, then the extinction equilibrium is locally asymptotically stable ${ }^{1}$, which threatens the model population with (asymptotic) extinction. If at least one eigenvalue is outside the complex unit circle, then the extinction equilibrium is unstable, which opens the possibility of population persistence. The nature of the bifurcation that occurs when the extinction equilibrium loses stability ${ }^{2}$ forms a fundamental bifurcation theorem in population dynamics. We describe this theorem below (Theorem 1).

In the matrix model (1), the vital rates and transitions modeled by the entries $p_{i j}(\hat{x})$ of the projection matrix $P(\hat{x})$ change temporally only due to changes in the demographic vector $\hat{x}=\hat{x}(t)$. There are, of course, numerous other reasons why these vital rates and transitions might change in time, for example, they might fluctuate randomly due to demographic or environmental stochasticity or periodically due to regular environmental oscillations (seasonal, monthly or daily fluctuations). Another reason these vital rates and transitions might change in time is that they are subject to selective pressures from Darwinian evolution. Our goal in this paper is to investigate an extension of the fundamental bifurcation theorem for the non-evolutionary model (1), as given in Theorem 1 below, to an evolutionary game theoretic version of (1). We describe the evolutionary model in Section 3, study the stability of an extinction equilibrium in Section 4, and in Section 5 determine the nature of the bifurcation that occurs when extinction stability is lost. In Section 6 an application

\footnotetext{
${ }^{1}$ An equilibrium $\hat{x}$ is locally stable if given any $\varepsilon>0$ there exists a $\delta>0$ such that for any initial condition satisfying $|\hat{x}(0)-\hat{x}|<\delta$ it follows that the solution satisfies $|\hat{x}(t)-\hat{x}|<\varepsilon$ for all $t \in N_{0}$. An equilibrium is a local attractor if there exists a $\delta_{0}>0$ such that $|\hat{x}(0)-\hat{x}|<\delta$ implies $\lim _{t \rightarrow+\infty} \hat{x}(t)=\hat{x}$. An equilibrium is locally asymptotically stable if it is both locally stable and a local attractor.

${ }^{2}$ Throughout this paper stable (or stability) means local asymptotically stable (or local asymptotic stability). Unstable means not locally asymptotically stable.
} 
is made to an evolutionary version of a Ricker model with an added Allee component (low density positive feedback effect).

\section{A Bifurcation Theorem for the Matrix Model (1).}

We make the following assumptions on the entries $p_{i j}(\hat{x})$ in the projection matrix $P(\hat{x})$. Let $\Omega \subseteq \mathbb{R}^{m}$ denote an open neighborhood of $\hat{0} \in \mathbb{R}^{m}$ and $C^{2}\left(\Omega \rightarrow \overline{\mathbb{R}}_{+}\right)$denote the set of twice continuously differentiable functions that map $\Omega$ to $\overline{\mathbb{R}}_{+}$.

H1: $P(\hat{x})=\left[p_{i j}(\hat{x})\right]$ is primitive for all $\hat{x} \in \Omega$ and $p_{i j} \in C^{2}\left(\Omega \rightarrow \overline{\mathbb{R}}_{+}\right)$.

Recall that a nonnegative matrix (i.e. one all of whose entries are nonnegative) is primitive if it is irreducible and has a strictly dominant eigenvalue. Perron-Frobenius theory implies that the spectral radius $\rho[A]$ of a primitive matrix $A$ is a strictly dominant, positive and simple eigenvalue which possesses a positive eigenvector in $\mathbb{R}_{+}^{m}$. Moreover, no other eigenvalue has a nonnegative eigenvector, i.e. an eigenvector in $\overline{\mathbb{R}}_{+}^{m}$. See [3]. We denote the strictly dominant eigenvalue of $P(\hat{x})$ by

$$
r(\hat{x}):=\rho[P(\hat{x})] .
$$

Observe that $r(\cdot) \in C^{2}\left(\Omega \rightarrow \mathbb{R}_{+}^{1}\right)$. The number $r(\hat{0})$ is the inherent growth rate of the population (the growth rate in the absence of density effects). For notational simplicity we denote this number by

$$
r_{0}:=r(\hat{0})
$$

For our purposes, we normalize the entries of $P$ in a way that

$$
P(\hat{x})=r_{0} Q(\hat{x})
$$

where the normalized matrix $Q(\hat{x})=\left[q_{i j}(\hat{x})\right]$ satisfies H1 and

$$
\rho[Q(\hat{0})]=1 .
$$

Then the matrix equation (1) becomes

$$
\hat{x}(t+1)=r_{0} Q(\hat{x}(t)) \hat{x}(t), \quad t \in \mathbb{N}_{0} .
$$

We denote the entries of the matrix $Q(\hat{x})$ by $q_{i j}(\hat{x})$.

Definition 1 We say that a pair $\left(r_{0}, \hat{x}\right) \in R \times \Omega$ is an equilibrium pair of (2) (or equivalently of (1)) if $\hat{x}=r_{0} Q(\hat{x}) \hat{x}$. Observe that $\left(r_{0}, \hat{0}\right)$ is an equilibrium pair for every $r_{0} \in R$; we call $\left(r_{0}, \hat{0}\right)$ an extinction equilibrium pair. An equilibrium pair $\left(r_{0}, \hat{x}\right)$ is a positive equilibrium pair if $\hat{x} \in R_{+}^{m}$ and it is stable if $\hat{x}$ is a locally asymptotically stable equilibrium of (2) (equivalently (1)).

We need the quantity

$$
\kappa:=-\hat{w}_{L}^{T}\left[\nabla_{\hat{x}}^{0} q_{i j}^{T} \hat{w}_{R}\right] \hat{w}_{R}
$$

where $T$ denotes transposition, the gradient $\nabla_{\hat{x}}$ of $q_{i j}(\hat{x})$ with respect to $\hat{x}$ is a column $m$-vector, and $\nabla_{\hat{x}}^{0} q_{i j}^{T}$ denotes the transpose of the gradient evaluated at the bifurcation point $\left(r_{0}, \hat{x}\right)=(1, \hat{0})$. With this superscript notational convention, we can equivalently write

$$
\kappa=-\hat{w}_{L}^{T}\left[\nabla_{\hat{x}}^{0} p_{i j}^{T} \hat{w}_{R}\right] \hat{w}_{R} .
$$


Here the vectors $\hat{w}_{L}^{T}$ and $\hat{w}_{R}$ are the (positive) left and right eigenvectors of $Q(\hat{0})$ (equivalently of $P(\hat{0})$ when $\left.r_{0}=1\right)$ associated with eigenvalue 1 , normalized so that

$$
\hat{w}_{L}^{T} \hat{w}_{R}=1 .
$$

Note that $\left[\nabla_{\hat{x}}^{0} p_{i j}^{T} \hat{w}_{R}\right]$ is an $m \times m$ matrix. The derivative $\partial_{x_{k}}^{0} p_{i j}$ measures the effect that an increase in the density of class $k$ has on the entry $p_{i j}$ of the population projection matrix $P$ (at low population density). The number $\hat{w}_{L}^{T}\left[\nabla_{\hat{x}}^{0} p_{i j}^{T} \hat{w}_{R}\right] \hat{w}_{R}$ is a weighted sum (with positive coefficients) of all density effects on all entries $p_{i j}$. This number therefore represents a summary measure of the effects that (low level) class densities has on the population (as does $\kappa$, the minus sign being introduced only for notational convenience in Theorem 1.)

From the linearization principle and from Theorems 1.2.4 and 1.2.5 in [7] we have the following result.

Theorem 1 Assume the matrix $P(\hat{x})$ in (1) satisfies $H 1$.

(a) The extinction equilibrium $\left(r_{0}, \hat{0}\right)$ is stable for $r_{0}<1$ and is unstable for $r_{0}>1$.

(b) There exists a continuum $\mathcal{C}$ of positive equilibrium pairs $\left(r_{0}, \hat{x}\right) \in \mathbb{R}_{+} \times \mathbb{R}_{+}^{m}$ of the matrix equation (1) which bifurcates from $(1, \hat{0})$ (i.e. contains the extinction pair (1,0) in its closure). Near the bifurcation point, the positive equilibrium pairs on $C$ have the parameterization

$$
\begin{aligned}
\hat{x}(\varepsilon) & =\hat{w}_{R} \varepsilon+\mathcal{O}\left(\varepsilon^{2}\right) \\
r_{0}^{*}(\varepsilon) & =1+\kappa \varepsilon+\mathcal{O}\left(\varepsilon^{2}\right)
\end{aligned}
$$

for $\varepsilon \gtrsim 0$.

(c) We say the bifurcation of positive equilibria is forward (respectively, backward) if, in a neighborhood of $(1, \hat{0})$, the positive equilibrium pairs on $\mathcal{C}$ are such that $r_{0}>1$ (respectively, $\left.r_{0}<1\right)$. If $\kappa>0$ then the bifurcation of $\mathcal{C}$ at $(1, \hat{0})$ is forward and the equilibrium pairs on $\mathcal{C}$ in a neighborhood of $(1, \hat{0})$ are (locally asymptotically) stable. If $\kappa<0$ then the bifurcation is backward and the equilibrium pairs on $\mathcal{C}$ in a neighborhood of $(1, \hat{0})$ are unstable.

Note how, in this Theorem, the direction of the bifurcation determines the stability of the bifurcating equilibria. A forward bifurcation, occurring when the extinction equilibrium loses its stability as $r_{0}$ increases through 1 (removing the threat of extinction), creates stable positive (nonextinction) equilibrium states.

Theorem 1 asserts stability or instability of the bifurcating positive equilibria $\mathcal{C}$ locally only, i.e. for equilibrium pairs on $\mathcal{C}$ near the extinction equilibrium $(1, \hat{0})$ only. However, the continuum $\mathcal{C}$ is known to exist globally in the sense that it connects to the boundary of the set on which the matrix model is defined, i.e., it connects to the set $\{+\infty\} \times\left(\partial \Omega \cap \mathbb{R}_{+}^{m}\right)$, where $\partial \Omega$ denotes the boundary of $\Omega$. In most applications, $\Omega$ includes the closure $\overline{\mathbb{R}}_{+}^{m}$ of the positive cone, which implies that either the component $r_{0}$ is unbounded or the norm $|\hat{x}|$ is unbounded in $\mathbb{R}_{+}$(or both). When $r_{0}$ is unbounded we have that there is at least one non-extinction equilibrium for each $r_{0}>1[7,8]$.

A derivative $\partial_{x_{k}} p_{i j}$ is often negative in population models because of an assumption that an increase in density $x_{k}$ will have a deleterious effect on some vital rate (birth rate, survival probability, growth rate, metabolic rates, and so on). These kinds of negative feedback phenomena are common in population models that describe density regulation mechanisms for population growth. If all the derivatives $\partial_{x_{k}}^{0} p_{i j}$ are negative (or zero), that is to say, if all density effects in a model are negative feedback effects, then clearly $\kappa>0$ and the bifurcation of the continuum $\mathcal{C}$ is forward and hence stable. 
A positive derivative $\partial_{x_{k}}^{0} p_{i j}$ is called a component Allee effect [5]. Clearly, the existence of a component Allee effect is necessary for a backward bifurcation (i.e. for $\kappa<0$ ). If all component Allee effects are sufficiently large so that $\kappa<0$, then the bifurcation of positive equilibria at $(1, \hat{0})$ is backward and hence unstable. A common occurrence in this case is the creation of a strong Allee effect, i.e. the presence of two attractors, one of which is an extinction equilibrium and the other of which is positive. Thus, population survival is initial condition dependent. This scenario can only occur when $r_{0}<1$ and the extinction equilibrium is stable. A backward bifurcation does not create a stable positive equilibrium, however. A strong Allee effect usually arises in models with backward bifurcations. This is because it is usually assumed that negative feedback effects predominate at high densities (even if they do not at low densities) which has the consequence of "turning" the continuum $\mathcal{C}$ around at a critical (saddle-node bifurcation) value of $r_{0}<1$ with a concomitant stabilization of the positive equilibria. We will not pursue this phenomena here, which occurs outside a neighborhood of the bifurcation point. See [10].

In this paper we extend the fundamental bifurcation Theorem 1 to an evolutionary version of the matrix model (2) under the assumption that the projection matrix depends on a suite of phenotypic traits subject to natural selection. This generalizes the results in [6] where models with only a single trait are considered.

\section{Darwinian Dynamics with Multiple Evolutionary Traits}

We consider an evolutionary version of the matrix model (1) developed in [28]. In that modeling methodology a (focal) individual's vital rates, as described by the entries of the projection matrix, are influenced by a collection of scalar traits $\hat{v}=\left(v_{1}, \ldots, v_{n}\right)^{T}$ and the population means of these traits $\hat{u}=\left(u_{1}, \ldots, u_{n}\right)^{T}$. By this assumption, an individual's fitness depends on both its own suite of traits $\hat{v}$ and the traits possessed by other individuals in the population $\hat{u}$ (frequency dependence). We indicate this by the notation $P(\hat{x}, \hat{u}, \hat{v})$, which in turn implies that the spectral radius of $P(\hat{x}, \hat{u}, \hat{v})$ is also dependent on $\hat{v}$ and $\hat{u}$ :

$$
r(\hat{x}, \hat{u}, \hat{v}):=\rho[P(\hat{x}, \hat{u}, \hat{v})] .
$$

Darwinian dynamics track the dynamics of the structured population $\hat{x}(t)$ and the vector of population mean traits $\hat{u}(t)=\left(u_{1}(t), \ldots, u_{n}(t)\right)^{T}$, the latter by means of the assumption that changes in the mean trait are proportional to the fitness gradient of the focal individual $[1,13,2,16,17,22,28]$. We extend the resulting evolutionary matrix model, as found in [28], to include a vector of traits $\hat{v}=\left(v_{1}, \ldots, v_{n}\right)^{T}$. Different fitness functions can be found throughout the literature, but the most common choice is the exponential growth rate $\ln r$ [24]. Another choice used by some researchers is the net reproduction number $R_{0}(\hat{x}, \hat{u}, \hat{v})$. We use $\ln r(\hat{x}, \hat{u}, \hat{v})$, but note that by the results in [9] our results remain unchanged if $r(\hat{x}, \hat{u}, \hat{v})$ is replaced by $R_{0}(\hat{x}, \hat{u}, \hat{v})$.

The model equations for the coupled population and trait dynamics provided by evolutionary game theory are $[22,28]$

$$
\begin{aligned}
& \hat{x}(t+1)=\left.P(\hat{x}, \hat{u}, \hat{v})\right|_{(\hat{x}, \hat{u}, \hat{v}=(\hat{x}(t), \hat{u}(t), \hat{u}(t))} \hat{x}(t) \\
& \hat{u}(t+1)=\hat{u}(t)+\left.M \nabla_{\hat{v}} \ln r(\hat{x}, \hat{u}, \hat{v})\right|_{(\hat{x}, \hat{u}, \hat{v})=(\hat{x}(t), \hat{u}(t), \hat{u}(t))}
\end{aligned}
$$

where $M=\left(\sigma_{i j}\right)$ is a symmetric $n \times n$ variance-covariance matrix for trait evolution and the gradient 
$\nabla_{\hat{v}} \ln r(\hat{x}, \hat{u}, \hat{v})$ is a column n-vector, whose $i^{\text {th }}$ entry is

$$
\partial_{v_{i}} \ln r(\hat{x}, \hat{u}, \hat{v}):=\frac{\partial \ln r(\hat{x}, \hat{u}, \hat{v})}{\partial v_{i}} .
$$

The entry $\sigma_{i j}$ of $M, i \neq j$, is the covariance of the $i^{t h}$ phenotypic trait and the $j^{t h}$ phenotypic trait. The diagonal entries

$$
\sigma_{i}^{2}:=\sigma_{i i} \geq 0
$$

are the variances of the $i^{\text {th }}$ trait (from its mean $u_{i}$ ) occurring in the population at each time $t$ (which are assumed constant). We assume the usual conditions for a covariance matrix, namely that $M$ is positive semi-definite and symmetric. ${ }^{3}$ If the matrix $M$ is the null matrix, then no evolution occurs and $\hat{u}(t)$ remains constant for all $t$. In this case Theorem 1 holds when applied to (4) with the mean trait $\hat{u}(t) \equiv \hat{u}(0)$ held fixed.

We write (4) and (5) as

$$
\begin{aligned}
& \hat{x}(t+1)=P(\hat{x}(t), \hat{u}(t), \hat{u}(t)) \hat{x}(t) \\
& \hat{u}(t+1)=\hat{u}(t)+M \nabla_{\hat{v}} \ln r(\hat{x}(t), \hat{u}(t), \hat{u}(t))
\end{aligned}
$$

where we use the simplifying notation

$$
\begin{aligned}
P(\hat{x}(t), \hat{u}(t), \hat{u}(t)) & :=\left.P(\hat{x}, \hat{u}, \hat{v})\right|_{(\hat{x}, \hat{u}, \hat{v})=(\hat{x}(t), \hat{u}(t), \hat{u}(t))} \\
\nabla_{\hat{v}} \ln r(\hat{x}(t), \hat{u}(t), \hat{u}(t)) & :=\left.\left[\nabla_{\hat{v}} \ln r(\hat{x}, \hat{u}, \hat{v})\right]\right|_{(\hat{x}, \hat{u}, \hat{v})=(\hat{x}(t), \hat{u}(t), \hat{u}(t))} .
\end{aligned}
$$

Remark 1 We will need to differentiate functions of the three variables $(\hat{x}, \hat{u}, \hat{v})$ after letting $\hat{v}=\hat{u}$ with respect to the components $u_{i}$ of $\hat{u}$ and from them construct gradients and Jacobians with respect to $\hat{u}$. Such a derivative is the sum of the partial derivatives with respect to $u_{i}$ and $v_{i}$. For example, the derivative of $r(\hat{x}, \hat{u}, \hat{u}):=\left.r(\hat{x}, \hat{u}, \hat{v})\right|_{\hat{v}=\hat{u}}$ with respect to $u_{i}$ is

$$
\frac{\partial}{\partial u_{i}}\left[\left.r(\hat{x}, \hat{u}, \hat{v})\right|_{\hat{v}=\hat{u}}\right]+\frac{d}{d v_{i}}\left[\left.r(\hat{x}, \hat{u}, \hat{v})\right|_{\hat{v}=\hat{u}}\right]
$$

which we write as

$$
\frac{\partial r(\hat{x}, \hat{u}, \hat{u})}{\partial u_{i}}+\frac{\partial r(\hat{x}, \hat{u}, \hat{u})}{\partial v_{i}} .
$$

With this notation, the gradient of $r(\hat{x}, \hat{u}, \hat{u})$ with respect to the components $u_{i}$ of $\hat{u}$ constructed from these partial derivatives is

$$
\nabla_{\hat{u}} r(\hat{x}, \hat{u}, \hat{u})+\nabla_{\hat{v}} r(\hat{x}, \hat{u}, \hat{u}) .
$$

Let $V$ be an open connected set in $R^{n}$ and let $\Omega \subseteq R^{m}$ be an open set containing the origin $\hat{0} \in R^{m}$. We assume the following about the projection matrix $P(\hat{x}, \hat{u}, \hat{v})$ and the variance-covariance matrix $V$.

H2. $P(\hat{x}, \hat{u}, \hat{v})$ is primitive for $(\hat{x}, \hat{u}, \hat{v}) \in \Omega \times V \times V, p_{i j} \in C^{2}\left(\Omega \times V \times V \rightarrow \overline{\mathbb{R}}_{+}\right)$, $p_{i j}(\hat{x}, \hat{u}, \hat{v})=\tilde{p}_{i j}(\hat{v}) \bar{p}_{i j}(\hat{x}, \hat{u}, \hat{v})$ such that $\bar{p}_{i j}(\hat{0}, \hat{u}, \hat{v}) \equiv 1$, and $M$ is invertible.

\footnotetext{
${ }^{3} M$ is positive semi-definite means $\hat{v}^{T} M \hat{v} \geq 0$ for all $\hat{v} \in R^{m} . M$ is symmetric means $\sigma_{i j}=\sigma_{j i}$ for all $1 \leq i, j \leq m$.
} 
Remark 2 The assumption on $p_{i j}$ in H2 implies that trait frequency dependence has no effect in the absence of density effects. Specifically, $p_{i j}(\hat{0}, \hat{u}, \hat{v})=\tilde{p}_{i j}(\hat{v})$. A mathematical implication of this assumption is that all derivatives of $p_{i j}(\hat{0}, \hat{u}, \hat{v})$ with respect to components $u_{i}$ of $\hat{u}$ are identically equal to 0 for all $\hat{v}$ :

$$
\nabla_{\hat{u}} p_{i j}(\hat{0}, \hat{u}, \hat{v}) \equiv 0_{n}
$$

This means the inherent projection matrix $P(\hat{0}, \hat{u}, \hat{v})$ is independent of $\hat{u}$ and hence so is its dominant eigenvalue $r(\hat{0}, \hat{u}, \hat{v})$. Thus $\nabla_{\hat{u}} r(\hat{0}, \hat{u}, \hat{v}) \equiv 0_{n}$ for all $\hat{v}$ hence $\nabla_{\hat{u}}\left[\left.r(\hat{0}, \hat{u}, \hat{v})\right|_{\hat{v}=\hat{u}}\right] \equiv 0_{n}$. Using the notation convention in Remark 1 we have

$$
\nabla_{\hat{u}} r(\hat{0}, \hat{u}, \hat{u}) \equiv 0_{n} .
$$

Remark 3 The assumption on $M$ in H2, that it is invertible, is for example satisfied if traits are not strongly correlated.

Our approach is to consider the bifurcation of equilibria from an extinction equilibrium which, by definition, is an equilibrium $(\hat{x}, \hat{u})=(\hat{0}, \hat{u})$ of $(6)$. From $(6 \mathrm{~b})$ we find that $\left(\hat{0}, \hat{u}^{*}\right)$ is an equilibrium if an only if

$$
\nabla_{\hat{v}} r\left(\hat{0}, \hat{u}^{*}, \hat{u}^{*}\right)=\hat{0}_{n}
$$

(where $\hat{0}_{n}$ is the origin in $\mathbb{R}^{n}$ ), in which case we say $\hat{u}^{*}$ is a critical trait. As a bifurcation parameter we use the dominant eigenvalue of $P\left(\hat{0}, \hat{u}^{*}, \hat{u}^{*}\right)$, which we denote by

$$
r_{0}^{*}:=\rho\left[P\left(\hat{0}, \hat{u}^{*}, \hat{u}^{*}\right)\right]
$$

This is the inherent growth rate of the population when the trait is held fixed at the critical trait $\hat{u}=\hat{u}^{*}$. As in the non-evolutionary case, we normalize the entries of the projection matrix so that

$$
P(\hat{x}, \hat{u}, \hat{v})=r_{0}^{*} Q(\hat{x}, \hat{u}, \hat{v})
$$

where $Q$ satisfies $\mathrm{H} 2$ and

$$
\rho\left[Q\left(\hat{0}, \hat{u}^{*}, \hat{u}^{*}\right)\right]=1
$$

Letting

$$
\bar{r}(\hat{x}, \hat{u}, \hat{v}):=\rho[Q(\hat{x}, \hat{u}, \hat{v})]
$$

we have

$$
r(\hat{x}, \hat{u}, \hat{v})=r_{0}^{*} \bar{r}(\hat{x}, \hat{u}, \hat{v}), \quad \bar{r}\left(\hat{0}, \hat{u}^{*}, \hat{u}^{*}\right)=1 .
$$

The Darwinian equations (6) are now

$$
\begin{aligned}
& \hat{x}(t+1)=r_{0}^{*} Q(\hat{x}(t), \hat{u}(t), \hat{u}(t)) \hat{x}(t) \\
& \hat{u}(t+1)=\hat{u}(t)+\frac{1}{\bar{r}(\hat{x}, \hat{u}, \hat{u})} M \nabla_{\hat{v}} \bar{r}(\hat{x}, \hat{u}, \hat{u}) .
\end{aligned}
$$

Note that the bifurcation parameter $r_{0}^{*}$ does not appear in the trait equation (10b). This is because in the trait equation $(6 \mathrm{~b})$ we have

$$
\nabla_{\hat{v}} \ln r(\hat{x}(t), \hat{u}(t), \hat{u}(t))=\frac{1}{r(\hat{x}, \hat{u}, \hat{u})} \nabla_{\hat{v}} r(\hat{x}, \hat{u}, \hat{u})=\frac{1}{r_{0}^{*} \bar{r}(\hat{x}, \hat{u}, \hat{u})} r_{0}^{*} \nabla_{\hat{v}} \bar{r}(\hat{x}, \hat{u}, \hat{u})
$$


in which $r_{0}^{*}$ cancels.

We say that a pair $\left(r_{0}^{*},(\hat{x}, \hat{u})\right) \in \mathbb{R} \times(\Omega \times V)$ is an equilibrium pair if

$$
\begin{aligned}
\hat{x} & =r_{0}^{*} Q(\hat{x}, \hat{u}, \hat{u}) \hat{x} \\
\hat{0}_{n} & =\nabla_{\hat{v}} \bar{r}(\hat{x}, \hat{u}, \hat{u}) .
\end{aligned}
$$

Note that

$$
\nabla_{\hat{v}} \bar{r}(\hat{x}, \hat{u}, \hat{u})=\hat{0}_{n} \text { if and only if } \nabla_{\hat{v}} r(\hat{x}, \hat{u}, \hat{u})=\hat{0}_{n} .
$$

Definition 2 We say an equilibrium pair $\left(r_{0}^{*},(\hat{x}, \hat{u})\right)$ is a positive equilibrium if $\hat{x} \in \mathbb{R}_{+}^{m}$. An extinction equilibrium pair is an equilibrium pair of the form $\left(r_{0}^{*},(\hat{0}, \hat{u})\right)$.

Observe that $\left(r_{0}^{*},(\hat{0}, \hat{u})\right)$ is an extinction equilibrium pair if and only if $\hat{u}=\hat{u}^{*}$ is a critical trait and, conversely, if $\hat{u}=\hat{u}^{*}$ is a critical trait, then $\left(r_{0}^{*},\left(\hat{0}, \hat{u}^{*}\right)\right)$ is an extinction equilibrium pair for all values of $r_{0}^{*}$.

\section{Stability of extinction equilibria}

We want to analyze the stability properties of an extinction equilibrium pair.

Definition 3 We say that an equilibrium pair $\left(r_{0}^{*},(\hat{x}, \hat{u})\right)$ is stable if $(\hat{x}, \hat{u})$ is (locally asymptotically) stable as an equilibrium of the Darwinian dynamics (6).

To use the Linearization Principle, we compute the Jacobian matrix for the system (10a)-(10b)

$$
\mathcal{J}\left(r_{0}^{*}, \hat{x}, \hat{u}\right)=\left(\begin{array}{cc}
r_{0}^{*} J(\hat{x}, \hat{u}, \hat{u}) & r_{0}^{*} \Psi(\hat{x}, \hat{u}, \hat{u}) \\
\Upsilon(\hat{x}, \hat{u}, \hat{u}) & \Phi(\hat{x}, \hat{u}, \hat{u})
\end{array}\right)
$$

where $J(\hat{x}, \hat{u}, \hat{u})$ is the $m \times m$ Jacobian matrix of $Q(\hat{x}, \hat{u}, \hat{u}) \hat{x}$ with respect to $\hat{x}$ and $\Psi(\hat{x}, \hat{u}, \hat{u})$ is the $m \times n$ matrix whose $n$ columns are

$$
\partial_{u_{i}} Q(\hat{x}, \hat{u}, \hat{u}) \hat{x}+\partial_{v_{i}} Q(\hat{x}, \hat{u}, \hat{u}) \hat{x}, \quad i=1,2, \cdots, n .
$$

The dynamics of the $i^{\text {th }}$ mean trait $u_{i}$ are given by

$$
u_{i}(t+1)=u_{i}(t)+\sum_{k=1}^{n} \sigma_{i k} \partial_{v_{k}} \ln \bar{r}(\hat{x}, \hat{u}, \hat{u}) .
$$

and therefore $\Upsilon(\hat{x}, \hat{u}, \hat{u})$ is the $n \times m$ matrix whose $i^{t h}$ row $(i=1,2, \cdots, n)$ is the transpose of the gradient

$$
\nabla_{\hat{x}} \sum_{k=1}^{n} \sigma_{i k} \partial_{v_{k}} \ln \bar{r}(\hat{x}, \hat{u}, \hat{u})=\left.\sum_{k=1}^{n} \sigma_{i k} \nabla_{\hat{x}} \partial_{v_{k}} \ln \bar{r}(\hat{x}, \hat{u}, \hat{v})\right|_{\hat{v}=\hat{u}}
$$

and

$$
\Phi(\hat{x}, \hat{u}, \hat{u})=I_{n \times n}+M H(\hat{x}, \hat{u}, \hat{u})
$$

where $H(\hat{x}, \hat{u}, \hat{u})$ is a $n \times n$ matrix whose $k j^{t h}$ entry is the $u_{k}$ derivative of $\partial_{v_{j}} \ln \bar{r}(\hat{x}, \hat{u}, \hat{u})$, i.e.

$$
H(\hat{x}, \hat{u}, \hat{u}):=\left[\left.\partial_{u_{k} v_{j}} \ln \bar{r}(\hat{x}, \hat{u}, \hat{v})\right|_{\hat{v}=\hat{u}}+\left.\partial_{v_{k} v_{j}} \ln \bar{r}(\hat{x}, \hat{u}, \hat{v})\right|_{\hat{v}=\hat{u}}\right]
$$


By assumption $\mathrm{H} 2$, the projection matrix $P(\hat{0}, \hat{u}, \hat{v})=\left[\tilde{p}_{i j}(\hat{v})\right]$ is independent of $\hat{u}$ and as a result

$$
\left.\partial_{u_{k} v_{j}} \ln \bar{r}(\hat{x}, \hat{u}, \hat{v})\right|_{(\hat{x}, \hat{u}, \hat{v})=(\hat{0}, \hat{u}, \hat{u})} \equiv 0
$$

for all $\hat{u}$. It follows that

$$
H\left(\hat{0}, \hat{u}^{*}, \hat{u}^{*}\right)=\left[\partial_{v_{k} v_{j}}^{0} \ln \bar{r}\right]
$$

where we have adopted the superscript notation

$$
\partial_{v_{k} v_{j}}^{0} \ln \bar{r}:=\left.\partial_{v_{k} v_{j}} \ln \bar{r}(\hat{x}, \hat{u}, \hat{v})\right|_{(\hat{x}, \hat{u}, \hat{v})=\left(\hat{0}, \hat{u}^{*}, \hat{u}^{*}\right)} .
$$

Thus, the matrix $H\left(\hat{0}, \hat{u}^{*}, \hat{u}^{*}\right)$ is the Hessian of $\ln \bar{r}(\hat{x}, \hat{u}, \hat{v})$ with respect to $\hat{v}$ evaluated at $(\hat{x}, \hat{u}, \hat{v})=$ $\left(\hat{0}, \hat{u}^{*}, \hat{u}^{*}\right)$.

The Jacobian $\mathcal{J}$ evaluated at an extinction equilibrium pair $\left(r_{0}^{*},(\hat{x}, \hat{u})\right)=\left(r_{0}^{*},\left(\hat{0}, \hat{u}^{*}\right)\right)$ is

$$
\mathcal{J}\left(r_{0}^{*}, \hat{0}, \hat{u}^{*}\right)=\left(\begin{array}{cc}
r_{0}^{*} J\left(\hat{0}, \hat{u}^{*}, \hat{u}^{*}\right) & 0_{m \times n} \\
\Upsilon\left(\hat{0}, \hat{u}^{*}, \hat{u}^{*}\right) & \Phi\left(\hat{0}, \hat{u}^{*}, \hat{u}^{*}\right)
\end{array}\right)=\left(\begin{array}{cc}
r_{0}^{*} Q\left(\hat{0}, \hat{u}^{*}, \hat{u}^{*}\right) & 0_{m \times n} \\
\Upsilon\left(\hat{0}, \hat{u}^{*}, \hat{u}^{*}\right) & \Phi\left(\hat{0}, \hat{u}^{*}, \hat{u}^{*}\right)
\end{array}\right)
$$

where $0_{m \times n}$ denotes the null matrix with dimension $m \times n$. We note that $J\left(\hat{0}, \hat{u}^{*}, \hat{u}^{*}\right)$ is the Jacobian with respect to $\hat{x}$ of (10a) when the trait is held fixed at $\hat{u}^{*}$. The eigenvalues of (15) are the $m$ eigenvalues of $r_{0}^{*} Q\left(\hat{0}, \hat{u}^{*}, \hat{u}^{*}\right)$ and the $n$ eigenvalues of $\Phi\left(\hat{0}, \hat{u}^{*}, \hat{u}^{*}\right)$.

Recall that $r_{0}^{*}$ is the (strictly) dominant eigenvalue of $r_{0}^{*} Q\left(\hat{0}, \hat{u}^{*}, \hat{u}^{*}\right)$. Thus, if $r_{0}^{*}>1$ the extinction equilibrium $\left(\hat{0}, \hat{u}^{*}\right)$ is unstable. On the other hand, if $r_{0}^{*}<1$ then stability (by linearization) is determined by the $n$ eigenvalues of $\Phi\left(\hat{0}, \hat{u}^{*}, \hat{u}^{*}\right)$. Using the linearization principle for discrete dynamical systems [14], we obtain the following result, which is an extension, for the evolutionary case with multiple traits, of the first statement in Theorem 1.

Theorem 2 Assume H2 holds and that $\hat{u}^{*} \in V$ is a critical trait.

(a) If $r_{0}^{*}<1$ and $\rho\left[\Phi\left(\hat{0}, \hat{u}^{*}, \hat{u}^{*}\right)\right]<1$, then the extinction equilibrium pair $\left(r_{0}^{*},\left(\hat{0}, \hat{u}^{*}\right)\right)$ is stable.

(b) If $r_{0}^{*}<1$ and $\rho\left[\Phi\left(\hat{0}, \hat{u}^{*}, \hat{u}^{*}\right)\right]>1$, then the extinction equilibrium pair $\left(r_{0}^{*},\left(\hat{0}, \hat{u}^{*}\right)\right)$ is unstable.

(c) If $r_{0}^{*}>1$, then the extinction equilibrium pair $\left(r_{0}^{*},\left(\hat{0}, \hat{u}^{*}\right)\right)$ is unstable.

To investigate the spectral radius $\rho\left[\Phi\left(\hat{0}, \hat{u}^{*}, \hat{u}^{*}\right)\right]$, which appears in Theorem 2, we make further assumptions on the matrix $M$.

H3. The variance-covariance matrix $M$ diagonally dominant: $\sigma_{i}^{2} \geq \sum_{j \neq i}\left|\sigma_{i j}\right|$.

In [27] it is shown, under assumption $H 3$, that $\rho\left[\Phi\left(\hat{0}, \hat{u}^{*}, \hat{u}^{*}\right)\right]<1$ if $H\left(\hat{0}, \hat{u}^{*}, \hat{u}^{*}\right)$ is negative definite and that $\rho\left[\Phi\left(\hat{0}, \hat{u}^{*}, \hat{u}^{*}\right)\right]>1$ if $H\left(\hat{0}, \hat{u}^{*}, \hat{u}^{*}\right)$ is positive semi-definite or indefinite provided the variances $\sigma_{i}^{2}$ are small.

Corollary 1 Assume H2 and H3 hold and that $\hat{u}^{*} \in V$ is a critical trait. If the variances $\sigma_{i}^{2}$ are small, then the extinction equilibrium pair $\left(r_{0}^{*},\left(\hat{0}, \hat{u}^{*}\right)\right)$ is

(a) stable if $r_{0}^{*}<1$ and the Hessian (14) is negative definite;

(b) unstable if $r_{0}^{*}>1$ or if the Hessian (14) is either indefinite or positive semi-definite. 
With regard to the variances, the assumption $\sigma_{i}^{2}<1 / \rho\left[H\left(\hat{0}, \hat{u}^{*}, \hat{u}^{*}\right)\right]$ is sufficient in Corollary 1. We are particularly interested in the case when the extinction equilibrium $\left(r_{0}^{*},\left(\hat{0}, \hat{u}^{*}\right)\right)$ loses stability as $r_{0}^{*}$ increases through 1 . This occurs in Theorem 2 when $\rho\left[\Phi\left(\hat{0}, \hat{u}^{*}, \hat{u}^{*}\right)\right]<1$. It also occurs in Corollary 1 , when $H\left(\hat{0}, \hat{u}^{*}, \hat{u}^{*}\right)$ is negative definite. This suggests the possibility that the (transcritical) bifurcation occurring at $r_{0}^{*}=1$ can result in a branch of stable positive (nonextinction) equilibria. We address this question in Section 5 .

As an example consider the case when there is no covariant evolution of the traits (i.e. that the off diagonal terms in $M$ are all equal to 0 and the diagonal terms $\sigma_{i}^{2}$ are positive) and when

$$
\partial_{v_{i} v_{j}}^{0} \bar{r}=0 \text { for } i \neq j \text {, }
$$

so that traits evolve nearly independently in a neighborhood of $(\hat{x}, \hat{u})=\left(\hat{0}, \hat{u}^{*}\right)$. With these assumptions the matrix $\Phi\left(\hat{0}, \hat{u}^{*}, \hat{u}^{*}\right)$ is diagonal and its eigenvalues are $1+\sigma_{i}^{2} \partial_{v_{i} v_{i}}^{0} \bar{r}$. From Theorem 2 we obtain the following corollary.

Corollary 2 Assume HQ holds and that $\hat{u}^{*} \in V$ is a critical trait. Further assume $\sigma_{i j}=0$ and (16) for all $i \neq j$. The extinction equilibrium pair $\left(r_{0}^{*},\left(\hat{0}, \hat{u}^{*}\right)\right)$ is

(a) stable if $r_{0}^{*}<1$ and $\left|1+\sigma_{i}^{2} \partial_{v_{i} v_{i}}^{0} \bar{r}\right|<1$ for all $i$;

(b) unstable if $r_{0}^{*}>1$;

(c) unstable for any $r_{0}^{*}>0$ if $\left|1+\sigma_{i}^{2} \partial_{v_{i} v_{i}}^{0} \bar{r}\right|>1$ for at least one $i$.

Note. In Corollary 2(c) the extinction equilibrium pair $\left(r_{0}^{*},\left(\hat{0}, \hat{u}^{*}\right)\right)$ is unstable, for any value of $r_{0}^{*}$, if $\partial_{v_{i} v_{i}}^{0} \bar{r}>0$ for at least one $i$. On the other hand, if $\partial_{v_{i} v_{i}}^{0} \bar{r}<0$ for all values of $i$, then extinction equilibrium pair $\left(r_{0}^{*},\left(\hat{0}, \hat{u}^{*}\right)\right)$ is stable for $r_{0}^{*}<1$ and small variances $\sigma_{i}^{2}$.

\section{A Bifurcation Theorem for the Evolutionary Model (10)}

The loss of stability by the extinction equilibrium pair when $r_{0}^{*}$ increases through 1 suggests the possibility of a (transcritical) bifurcation at the value $r_{0}^{*}=1$. This is due to the fact that an eigenvalue of the Jacobian leaves the complex unit circle as $r_{0}^{*}$ increases through 1 . In this section we establish a bifurcation theorem for the evolutionary model (10).

We begin by assuming that $\hat{u}$ can be expressed as a function of $\hat{x}$ by means of the equilibrium equation (11b).

H4. Let $\hat{u}^{*} \in V$ be a critical trait. Assume there exists a function $\hat{\xi} \in C^{2}(N \rightarrow V)$, where $N$ is a open neighborhood of $\hat{0}$ in $\mathbb{R}^{m}$, such that $\hat{\xi}(\hat{0})=\hat{u}^{*}$ and $\nabla_{\hat{v}} \bar{r}(\hat{x}, \hat{\xi}(\hat{x}), \hat{\xi}(\hat{x}))=$ $\hat{0}_{n}$ for $\hat{x} \in N$.

Let $J_{\hat{u}}^{0}\left(\nabla_{\hat{v}} \bar{r}\right)$ and $J_{\hat{x}}^{0}\left(\nabla_{\hat{v}} \bar{r}\right)$ denote the Jacobian matrices of the gradient $\nabla_{\hat{v}} \bar{r}(\hat{x}, \hat{u}, \hat{u})$ with respect to $\hat{u}$ and $\hat{x}$ respectively evaluated at $(\hat{x}, \hat{u}, \hat{u})=\left(\hat{0}, \hat{u}^{*}, \hat{u}^{*}\right)$. The following assumption and the Implicit Function Theorem guarantee that H4 holds.

H5. Let $u^{*} \in V$ be a critical trait for which $J_{\hat{u}}^{0}\left(\nabla_{\hat{v}} \bar{r}\right)$ is a non-singular matrix.

Remark 4 The product rule (31) applied to

$$
\left.\nabla_{\hat{v}} \ln \bar{r}(\hat{x}, \hat{u}, \hat{v})\right|_{\hat{v}=\hat{u}}=\left.\frac{1}{\bar{r}} \nabla_{\hat{v}} \bar{r}(\hat{x}, \hat{u}, \hat{v})\right|_{\hat{v}=\hat{u}}
$$


evaluated at $\hat{x}=\hat{0}$ implies, together with $\bar{r}^{0}=1$ and $\nabla_{\hat{v}}^{0} \bar{r}=\hat{0}$ (by the definitikon of a critical trait), that $J_{\hat{u}}^{0} \nabla_{\hat{v}} \ln \bar{r}=J_{\hat{u}}^{0} \nabla_{\hat{v}} \bar{r}$ or

$$
J_{\hat{u}}^{0} \nabla_{\hat{v}} \ln \bar{r}=\left[\partial_{v_{k} v_{j}}^{0} \ln \bar{r}\right] .
$$

Thus, under assumption H2 we see (from (14)) that in H5

$$
J_{\hat{u}}^{0}\left(\nabla_{\hat{v}} \bar{r}\right)=H\left(\hat{0}, \hat{u}^{*}, \hat{u}^{*}\right) .
$$

Theorem 3 Assume $\hat{u}^{*} \in V$ is a critical trait. Assume H2 and H4 hold and that $\kappa^{*} \neq 0$.

(1) There exists a continuum $\mathcal{C}^{*}$ of positive equilibrium pairs $\left(r_{0}^{*},(\hat{x}, \hat{u})\right) \in \mathbb{R}_{+} \times\left(\mathbb{R}_{+}^{m} \times V\right)$ of (10) that bifurcates from the extinction pair $\left(1,\left(\hat{0}, \hat{u}^{*}\right)\right)$ (i.e. that contains the extinction pair in its closure).

(2) Assume H2 and H5 hold. In a neighborhood of $\left(1,\left(\hat{0}, \hat{u}^{*}\right)\right)$, the positive equilibrium pairs have the parametric representation

$$
\begin{aligned}
\hat{x}(\varepsilon) & =\hat{w}_{R} \varepsilon+\mathcal{O}\left(\varepsilon^{2}\right) \\
\hat{u}(\varepsilon) & =\hat{u}^{*}+\hat{u}_{1} \varepsilon+\mathcal{O}\left(\varepsilon^{2}\right) \\
r_{0}^{*}(\varepsilon) & =1+\kappa^{*} \varepsilon+\mathcal{O}\left(\varepsilon^{2}\right)
\end{aligned}
$$

for small $\varepsilon \gtrsim 0$ where $\hat{w}_{R}$ is a positive right eigenvector of $Q\left(\hat{0}, \hat{u}^{*}, \hat{u}^{*}\right)$ associated with eigenvalue 1 (equivalently of $P\left(\hat{0}, \hat{u}^{*}, \hat{u}^{*}\right)$ when $r_{0}^{*}=1$ ) and

$$
\begin{gathered}
\hat{u}_{1}:=-\left[J_{\hat{u}}^{0}\left(\nabla_{\hat{v}} \bar{r}\right)\right]^{-1} J_{\hat{x}}^{0}\left(\nabla_{\hat{v}} \bar{r}\right) \hat{w}_{R} . \\
\kappa^{*}:=-\hat{w}_{L}^{T}\left(\left[\nabla_{\hat{x}}^{0} q_{i j}^{T} \hat{w}_{R}\right]\right) \hat{w}_{R} .
\end{gathered}
$$

Furthermore, we have the following alternatives.

(a) If $\rho\left[\Phi\left(\hat{0}, \hat{u}^{*}, \hat{u}^{*}\right)\right]<1$ and $\kappa^{*}>0$, then the bifurcation of $\mathcal{C}^{*}$ is forward and the positive equilibrium pairs on $\mathcal{C}^{*}$ are stable.

(b) If $\rho\left[\Phi\left(\hat{0}, \hat{u}^{*}, \hat{u}^{*}\right)\right]<1$ and $\kappa^{*}<0$, then the bifurcation is backward and the positive equilibrium pairs on $\mathcal{C}^{*}$ are unstable.

(c) If $\rho\left[\Phi\left(\hat{0}, \hat{u}^{*}, \hat{u}^{*}\right)\right]>1$, then positive equilibrium pairs in the continuum $\mathcal{C}^{*}$ are unstable regardless of the direction of bifurcation.

Remark 5 Because $\kappa^{*}$ is calculated from evaluations at the bifurcation point $\left(r_{0}^{*},(\hat{x}, \hat{u})\right)=\left(1,\left(\hat{0}, \hat{u}^{*}\right)\right)$ and because only the sign of $\kappa^{*}$ is involved in determining the direction of bifurcation and stability, Theorem 3, parts (a) and (b), remains valid if in the formula (19) and in $\mathrm{H}_{4}$ and $\mathrm{H}_{5}$ we replace $q_{i j}$ by $p_{i j}$ and $\bar{r}$ by $r$.

Proof. (1) Under H4, the equilibrium equations (11) reduce to the single equation

$$
\hat{x}=r_{0}^{*} Q(\hat{x}, \hat{\xi}(\hat{x}), \hat{\xi}(\hat{x})) \hat{x}
$$

for $\hat{x} \in N$. Theorem 1 applies to this equation with matrix $Q(\hat{x}, \hat{\xi}(\hat{x}), \hat{\xi}(\hat{x}))$ in place of $Q(\hat{x})$ (and $N$ in place of $\Omega$ ). This results in the existence of a continuum $\mathcal{C}$ of positive solution pairs $\left(r_{0}^{*}, \hat{x}\right)$ of $(20)$ that bifurcates from $(1, \hat{0})$. The continuum $\mathcal{C}$ in turn gives rise to a continuum

$$
\mathcal{C}^{*}:=\left\{\left(r_{0}^{*},(\hat{x}, \hat{u})\right) \mid\left(r_{0}^{*}, \hat{x}\right) \in \mathcal{C}, \hat{u}=\hat{\xi}(\hat{x})\right\}
$$


of equilibrium pairs $\left(r_{0}^{*},(\hat{x}, \hat{u})\right)$ of $(10)$ that bifurcates from the extinction equilibrium $\left(1,\left(\hat{0}, \hat{u}^{*}\right)\right)$ at $r_{0}^{*}=1$

(2) The parameterization of $C$ Theorem 1 implies that, near the bifurcation point, the positive equilibrium pairs on the continuum $\mathrm{C}^{*}$ have a parameterization (17). The coefficient $\kappa^{*}$ is given by the formula (3) for $\kappa$ but with $p_{i j}(\hat{x})$ replaced by $p_{i j}(\hat{x}, \hat{\xi}(\hat{x}), \hat{\xi}(\hat{x}))$. To make this calculation we note that the coefficient $\hat{u}_{1}=\nabla^{0} \hat{\xi}^{T} \hat{w}_{R}$ can be calculated by an implicit differentiation of the equation $\nabla_{\hat{v}} \bar{r}(\hat{x}(\varepsilon), \hat{\xi}(\hat{x}(\varepsilon)), \hat{\xi}(\hat{x}(\varepsilon)))=\hat{0}_{n}$ with respect to $\varepsilon$ and a subsequent evaluation at $\varepsilon=0$. From this calculation we obtain (18). From (3) and Lemma 1 in the Appendix, but with $p_{i j}(\hat{x})$ replaced by $p_{i j}(\hat{x}, \hat{\xi}(\hat{x}), \hat{\xi}(\hat{x}))$, we obtain (19).

To investigate the stability of bifurcating positive equilibrium pairs we make use of the parametrization (17) which allows us to parameterize by $\varepsilon$ the Jacobian $J(r, \hat{x}, \hat{u})$ when it is evaluated at the equilibria (17) and, subsequently, to parameterize this Jacobian's eigenvalues by $\varepsilon$. From this parameterization we can approximate the eigenvalues of the Jacobian for $\varepsilon \gtrsim 0$.

At $\varepsilon=0$ the eigenvalues of the Jacobian $J\left(1, \hat{0}, \hat{u}^{*}\right)$ are the eigenvalues of $J\left(\hat{0}, \hat{u}^{*}, \hat{u}^{*}\right)$ and the eigenvalues of $\Phi\left(\hat{0}, \hat{u}^{*}, \hat{u}^{*}\right)$. The spectrum of the Jacobian $J\left(r_{0}^{*}(\varepsilon), \hat{x}(\varepsilon), \hat{u}(\varepsilon)\right)$ approaches, by continuity, the spectrum of $J\left(1, \hat{0}, \hat{u}^{*}\right)$ as $\varepsilon$ tends to 0 . Therefore, if $\rho\left[\Phi\left(\hat{0}, \hat{u}^{*}, \hat{u}^{*}\right)\right]>1$ then for $\varepsilon \gtrsim 0$ the Jacobian $J\left(r_{0}^{*}(\varepsilon), \hat{x}(\varepsilon), u(\varepsilon)\right)$ also has spectral radius greater than 1 . Consequently, the positive equilibria are unstable near the bifurcation point. This establishes 2(c).

Suppose $\rho\left[\Phi\left(\hat{0}, \hat{u}^{*}, \hat{u}^{*}\right)\right]<1$. Since the strictly dominant eigenvalue of $J\left(\hat{0}, \hat{u}^{*}, \hat{u}^{*}\right)$ is 1 , it follows that the dominant eigenvalue of $\mathcal{J}\left(1, \hat{0}, u^{*}\right)$ is 1 . To determine stability of the bifurcating positive equilibria, by means of the linearization principle, we must investigate if the dominant eigenvalue of $\mathcal{J}\left(r_{0}^{*}(\varepsilon), \hat{x}(\varepsilon), \hat{u}(\varepsilon)\right)$, which equals 1 when $\varepsilon=0$, is greater or less than 1 for $\varepsilon \gtrsim 0$. Let

$$
\mu(\varepsilon)=1+\mu_{1} \varepsilon+\mathcal{O}\left(\varepsilon^{2}\right)
$$

denote the dominant eigenvalue of $\mathcal{J}\left(r_{0}^{*}(\varepsilon), \hat{x}(\varepsilon), \hat{u}(\varepsilon)\right)$. Whether $\mu(\varepsilon)$ is less than or greater than 1 for $\varepsilon \gtrsim 0$, and hence whether the bifurcating positive equilibria are stable or unstable near the bifurcation point, can be determined by the sign of $\mu_{1}$ : for $\varepsilon \gtrsim 0$, the bifurcating positive equilibria are stable if $\mu_{1}<0$ and unstable if $\mu_{1}>0$.

To calculate a formula for $\mu_{1}$ we begin by letting

$$
\hat{W}_{R}(\varepsilon)=\hat{W}_{R 0}+\hat{W}_{R 1} \varepsilon+\mathcal{O}\left(\varepsilon^{2}\right)
$$

denote a right eigenvector of the Jacobian $\mathcal{J}$ associated with the dominant eigenvalue $\mu(\varepsilon)$, so that

$$
\mathcal{J}\left(r_{0}^{*}(\varepsilon), \hat{x}(\varepsilon), \hat{u}(\varepsilon)\right) \hat{W}_{R}(\varepsilon)=\mu(\varepsilon) \hat{W}_{R}(\varepsilon) .
$$

Setting $\varepsilon=0$ in (21) we obtain

$$
\mathcal{J}\left(1, \hat{0}, \hat{u}^{*}\right) \hat{W}_{R 0}=\hat{W}_{R 0} \text {. }
$$

We can write

$$
\hat{W}_{R 0}=\left(\begin{array}{c}
\hat{w}_{R 0}^{m} \\
\hat{w}_{R 0}^{n}
\end{array}\right)
$$

where $\hat{w}_{R 0}^{m}$ and $\hat{w}_{R 0}^{n}$, are column vectors with $m$ and $n$ entries respectively. The vector $\hat{w}_{R 0}^{m}$ is a right eigenvector of $J\left(\hat{0}, \hat{u}^{*}, \hat{u}^{*}\right)=Q\left(\hat{0}, \hat{u}^{*}, \hat{u}^{*}\right)$ associated with the eigenvalue 1 and consequently $\hat{w}_{R 0}^{m}=\hat{w}_{R}$. A calculation shows

$$
\hat{W}_{R 0}=\left(\begin{array}{c}
\hat{w}_{R} \\
\hat{u}_{1}
\end{array}\right) .
$$


The vector $\hat{W}_{L 0}^{T}$ where

$$
\hat{W}_{L 0}=\left(\begin{array}{c}
\hat{w}_{L} \\
\hat{0}_{n}
\end{array}\right)
$$

is a left eigenvector of $\mathcal{J}\left(1, \hat{0}, \hat{u}^{*}\right)$ (where $\hat{w}_{L}^{T}$ is a left eigenvector of $J\left(\hat{0}, \hat{u}^{*}, \hat{u}^{*}\right)$ ). Note that $\hat{W}_{L 0}^{T} \hat{W}_{R 0}=\hat{w}_{L}^{T} \hat{w}_{R}=1$.

If we differentiate (21) with respect to $\varepsilon$ and set $\varepsilon=0$ in the result, we obtain

$$
\left(\mathcal{J}\left(1, \hat{0}, \hat{u}^{*}\right)-I_{m+n}\right) \hat{W}_{R 1}=\mu_{1} \hat{W}_{R 0}-\left.\frac{d}{d \varepsilon} \mathcal{J}\left(r_{0}^{*}(\varepsilon), \hat{x}(\varepsilon), \hat{u}(\varepsilon)\right)\right|_{\varepsilon=0} \hat{W}_{R 0}
$$

where $I_{m+n}$ denotes the identity matrix of size $m+n$. According to Fredholm alternative, this equation is solvable for $\hat{W}_{R 1}$ if and only if the right hand side is orthogonal to the kernel of $\left(\mathcal{J}\left(1, \hat{0}, \hat{u}^{*}\right)-I_{m+n}\right)^{T}$. This kernel is spanned by the left eigenvectors of $\mathcal{J}\left(1, \hat{0}, \hat{u}^{*}\right)$ associated with the eigenvalue 1 . Therefore, the right hand side must be orthogonal to $\hat{W}_{L 0}$. This implies

$$
\begin{gathered}
\mathcal{J}\left(r_{0}^{*}, \hat{x}, \hat{u}\right)=\left(\begin{array}{cc}
r_{0}^{*} J(\hat{x}, \hat{u}, \hat{u}) & r_{0}^{*} \Psi(\hat{x}, \hat{u}, \hat{u}) \\
\Upsilon(\hat{x}, \hat{u}, \hat{u}) & \Phi(\hat{x}, \hat{u}, \hat{u})
\end{array}\right) \\
\mu_{1}=\left.\hat{W}_{L 0}^{T} \frac{d}{d \varepsilon} \mathcal{J}\left(r_{0}^{*}(\varepsilon), \hat{x}(\varepsilon), \hat{u}(\varepsilon)\right)\right|_{\varepsilon=0} \hat{W}_{R 0} \\
=\left.\left(\begin{array}{c}
\hat{w}_{L} \\
\hat{0}_{n}
\end{array}\right)^{T} \frac{d}{d \varepsilon}\left(\begin{array}{cc}
r_{0}^{*}(\varepsilon) J(\hat{x}(\varepsilon), \hat{u}(\varepsilon), \hat{u}(\varepsilon)) & r_{0}^{*}(\varepsilon) \Psi(\hat{x}(\varepsilon), \hat{u}(\varepsilon), \hat{u}(\varepsilon)) \\
\Upsilon(\hat{x}(\varepsilon), \hat{u}(\varepsilon), \hat{u}(\varepsilon)) & \Phi(\hat{x}(\varepsilon), \hat{u}(\varepsilon), \hat{u}(\varepsilon))
\end{array}\right)\right|_{\varepsilon=0}\left(\begin{array}{c}
\hat{w}_{R} \\
\hat{u}_{1}
\end{array}\right)
\end{gathered}
$$

or

$$
\mu_{1}=\left.\hat{w}_{L}^{T} \frac{d}{d \varepsilon}\left[r_{0}^{*}(\varepsilon) J(\hat{x}(\varepsilon), \hat{u}(\varepsilon), \hat{u}(\varepsilon))\right]\right|_{\varepsilon=0} \hat{w}_{R}+\left.\hat{w}_{L}^{T} \frac{d}{d \varepsilon}\left[r_{0}^{*}(\varepsilon) \Psi(\hat{x}(\varepsilon), \hat{u}(\varepsilon), \hat{u}(\varepsilon))\right]\right|_{\varepsilon=0} \hat{u}_{1} .
$$

We consider the two terms in this sum one at a time. With regard to the first term in $\mu_{1}$, the product and chain rules imply

$$
\begin{aligned}
& \left.\hat{w}_{L}^{T} \frac{d}{d \varepsilon}\left[r_{0}^{*}(\varepsilon) J(\hat{x}(\varepsilon), \hat{u}(\varepsilon), \hat{u}(\varepsilon))\right]\right|_{\varepsilon=0} \hat{w}_{R} \\
& =\kappa^{*} \hat{w}_{L}^{T} Q\left(\hat{0}, \hat{u}^{*}, \hat{u}^{*}\right) \hat{w}_{R}+\left.\hat{w}_{L}^{T} \frac{d}{d \varepsilon} J(\hat{x}(\varepsilon), \hat{u}(\varepsilon), \hat{u}(\varepsilon))\right|_{\varepsilon=0} \hat{w}_{R} \\
& =\kappa^{*}+\hat{w}_{L}^{T}\left(\left[\nabla_{\hat{x}}^{0} q_{i j}^{T} \hat{w}_{R}\right]+\left[\nabla_{\hat{u}}^{0} q_{i j}^{T} \hat{u}_{1}\right]+\left[\nabla_{\hat{v}}^{0} q_{i j}^{T} \hat{u}_{1}\right]+\left[\partial_{x_{i}}^{0} q_{j} \hat{w}_{R}\right]\right) \hat{w}_{R}
\end{aligned}
$$

where we have defined the row vector

$$
\partial_{x_{i}}^{0} q_{j}:=\left[\begin{array}{llll}
\partial_{x_{i}}^{0} q_{j 1} & \partial_{x_{i}}^{0} q_{j 2} & \cdots & \partial_{x_{i}}^{0} q_{j m}
\end{array}\right] .
$$

With regard to the second term in $\mu_{1}$, we recall that $\Psi(\hat{x}, \hat{u}, \hat{u})$ is the $m \times n$ matrix whose $n$ columns are (12) and as a result, upon evaluation at the bifurcation point, the only contribution 
to the derivative in the second term arises from the derivatives of $\Psi(\hat{x}, \hat{u}, \hat{u})=\left[\psi_{i j}(\hat{x}, \hat{u}, \hat{u})\right]$ with respect to the components of $\hat{x}$. Therefore the second term in $\mu_{1}$ is

$$
\begin{aligned}
\left.\hat{w}_{L}^{T} \frac{d}{d \varepsilon}\left[r_{0}^{*}(\varepsilon) \Psi(\hat{x}(\varepsilon), \hat{u}(\varepsilon), \hat{u}(\varepsilon))\right]\right|_{\varepsilon=0} \hat{u}_{1} & =\hat{w}_{L}^{T}\left[\nabla_{\hat{x}}^{0} \psi_{i j}^{T} \hat{w}_{R}\right] \hat{u}_{1} \\
& =\hat{w}_{L}^{T}\left(\left[\partial_{u_{i}}^{0} q_{j} \hat{w}_{R}\right]+\left[\partial_{v_{i}}^{0} q_{j} \hat{w}_{R}\right]\right) \hat{u}_{1}
\end{aligned}
$$

where we have defined the row vectors

$$
\partial_{u_{i}}^{0} q_{j}:=\left[\begin{array}{llll}
\partial_{u_{i}}^{0} q_{j 1} & \partial_{u_{i}}^{0} q_{j 2} & \cdots & \partial_{u_{i}}^{0} q_{j m}
\end{array}\right], \quad \partial_{v_{i}}^{0} q_{j}:=\left[\begin{array}{llll}
\partial_{v_{i}}^{0} q_{j 1} & \partial_{v_{i}}^{0} q_{j 2} & \cdots & \partial_{v_{i}}^{0} q_{j m}
\end{array}\right] .
$$

H2 implies $\partial_{u_{k}}^{0} q_{i j}=0$ for all $u_{k}$ and all $i, j$, and

$$
\mu_{1}=\kappa^{*}+\hat{w}_{L}^{T}\left[\nabla_{\hat{x}}^{0} q_{i j}^{T} \hat{w}_{R}\right] \hat{w}_{R}+\hat{w}_{L}^{T}\left[\nabla_{\hat{v}}^{0} q_{i j}^{T} \hat{u}_{1}\right] \hat{w}_{R}+\hat{w}_{L}^{T}\left[\partial_{x_{i}}^{0} q_{j} \hat{w}_{R}\right] \hat{w}_{R}+\hat{w}_{L}^{T}\left[\partial_{v_{i}}^{0} q_{j} \hat{w}_{R}\right] \hat{u}_{1} .
$$

Noting that

$$
\hat{w}_{L}^{T}\left[\nabla_{\hat{x}}^{0} q_{i j}^{T} \hat{w}_{R}\right] \hat{w}_{R}=\hat{w}_{L}^{T}\left[\partial_{x_{i}}^{0} q_{j} \hat{w}_{R}\right] \hat{w}_{R}, \quad \hat{w}_{L}^{T}\left[\nabla_{\hat{v}}^{0} q_{i j}^{T} \hat{u}_{1}\right] \hat{w}_{R}=\hat{w}_{L}^{T}\left[\partial_{v_{i}}^{0} q_{j} \hat{w}_{R}\right] \hat{u}_{1}
$$

we have

$$
\mu_{1}=\kappa^{*}+2\left(\hat{w}_{L}^{T}\left[\nabla_{\hat{x}}^{0} q_{i j}^{T} \hat{w}_{R}\right] \hat{w}_{R}+\hat{w}_{L}^{T}\left[\nabla_{\hat{v}}^{0} q_{i j}^{T} \hat{u}_{1}\right] \hat{w}_{R}\right) .
$$

By Lemma 1 in the Appendix we get

$$
\mu_{1}=\kappa^{*}+2 \hat{w}_{L}^{T}\left[\nabla_{\hat{x}}^{0} q_{i j}^{T} \hat{w}_{R}\right] \hat{w}_{R}
$$

which, by (18), implies $\mu_{1}=-\kappa^{*}$.As a result, $\kappa^{*}>0$ implies both that the bifurcation is forward and that the bifurcating positive equilibria are stable for $\varepsilon \gtrsim 0$. On the other hand, $\kappa^{*}<0$ implies that the bifurcation is backward and that the bifurcating positive equilibria are unstable for $\varepsilon \gtrsim 0$.

From (13) we obtain (as in [27]) the following corollary of Theorem 3.

Corollary 3 Assume H2, H3 and H5 hold and that $\hat{u}^{*} \in V$ is a critical trait. Let $\mathcal{C}^{*}$ be the continuum of positive equilibrium pairs that bifurcates from the extinction pair $\left(1,\left(\hat{0}, \hat{u}^{*}\right)\right)$ guaranteed by Theorem 3. If the variances $\sigma_{i}^{2}$ are small, then in a neighborhood of the bifurcation point $\left(1,\left(\hat{0}, \hat{u}^{*}\right)\right)$ we have the following alternatives.

(a) The bifurcation of $\mathcal{C}^{*}$ is forward and stable if the Hessian (14) is negative definite and $\kappa^{*}>0$.

(b) The bifurcation of $\mathcal{C}^{*}$ is backward and unstable if the Hessian (14) is negative definite and $\kappa^{*}<0$.

(c) The positive equilibrium pairs in the continuum $\mathcal{C}^{*}$ are unstable if the Hessian (14) is positive semi-definite or indefinite (regardless of the direction of bifurcation).

For the case of no trait covariance considered in Corollary 2, we obtain the following result from Corollary 3.

Corollary 4 Assume H2 and H5 hold and that $\hat{u}^{*} \in V$ is a critical trait. Further assume $\sigma_{i j}=0$ and (16) for all $i \neq j$. Let $\mathcal{C}^{*}$ be the continuum of positive equilibrium pairs that bifurcates from the extinction pair $\left(1,\left(\hat{0}, \hat{u}^{*}\right)\right)$ guaranteed by Theorem 3. Then in a neighborhood of the bifurcation point $\left(1,\left(\hat{0}, \hat{u}^{*}\right)\right)$ we have the following alternatives. 
(a) The bifurcation of $\mathcal{C}^{*}$ is forward and stable if $\kappa^{*}>0$ and $\left|1+\sigma_{i}^{2} \partial_{v_{i} v_{i}}^{0} \bar{r}\right|<1$ for all $i$.

(b) The bifurcation of $\mathcal{C}^{*}$ is backward and unstable if $\kappa^{*}<0$ and $\left|1+\sigma_{i}^{2} \partial_{v_{i} v_{i}}^{0} \bar{r}\right|<1$ for all $i$.

(c) The positive equilibrium pairs in the continuum $\mathcal{C}^{*}$ are unstable if $\left|1+\sigma_{i}^{2} \partial_{v_{i} v_{i}}^{0} \bar{r}\right|>1$ for at least one $i$ (regardless of the direction of bifurcation).

Note. In Corollary 4 we see that the positive equilibrium pairs in the continuum $\mathcal{C}^{*}$ are unstable if $\partial_{v_{i} v_{i}}^{0} \bar{r}>0$ for at least one $i$. On the other hand, if $\partial_{v_{i} v_{i}}^{0} \bar{r}<0$ for all values of $i$, then the positive equilibrium pairs in the continuum $\mathcal{C}^{*}$ are stable if $\kappa^{*}>0$ and the variances $\sigma_{i}^{2}$ are small enough so that $\left|1+\sigma_{i}^{2} \partial_{v_{i} v_{i}}^{0} \bar{r}\right|<1$, i.e. $\sigma_{i}^{2}<-2 / \partial_{v_{i} v_{i}}^{0} \bar{r}$.

\section{An Application}

Consider the single difference equation

$$
x(t+1)=b x(t) \exp (-c x(t)) \exp \left(-\frac{\alpha}{1+s x(t)}\right)
$$

with coefficients $b, c>0$ and $\alpha, s \geq 0$. When $\alpha=0$ this map is the famous Ricker equation which is one of the most well known equations that incorporates negative effects that population density can have on population growth. Equation (22) is studied in [25] as a model equation that incorporates a positive effect of increased population density (a so-called component Allee effect [5]) in the presence of a predator. This is the well-known predator-saturation effect in ecology and is one of the most commonly attributed causes of Allee effects [12], [5].

The factor $\exp (-\alpha /(1+s x))$ in $(22)$ is an increasing function of $x$ and represents the probability of escaping predation. We can interpret $\alpha$ as the intensity of predation and $s$ a measure of how effective the protection from predation attributed to population density $x$, which we will refer to as the predation protection factor. Re-writing (22) as

$$
x(t+1)=r_{0} \bar{r}(x) x(t)
$$

with

$$
r_{0}:=b \exp (-\alpha), \quad \bar{r}(x):=\exp \left(-c x(t)+\alpha \frac{s x(t)}{1+s x(t)}\right)
$$

we see that $r_{0}$ is the inherent (density-free) per capita birth rate, which equals $b$ in the absence of predation $\alpha=0$. This equation, studied in [25], not surprisingly can exhibit the same kind of period-doubling route-to-chaos as $r_{0}$ increases as does the famous Ricker equation when $\alpha=0$. (The right side of (23) defines a unimodal map.) The bifurcation that occurs at $r_{0}=1$ where the extinction equilibrium $x=0$ destabilizes is, according to Theorem 1, forward and therefore stable if $\kappa=c-\alpha s>0$. This inequality holds if the effect of predation, as measured by the product of the predation intensity $\alpha$ and (per capita) predation protection factor $s$, is small compared to that of the negative density effects measured by $c$. This occurs, of course, for the Ricker equation when $\alpha=0$.

On the other, if the reverse is true and the effect of predation $\alpha s$ is large compared to $c$, then by Theorem 1 the bifurcation at $r_{0}=1$ is backward and unstable. In this case, i.e. when $\alpha s>c$, we can also say some things about the bifurcating continuum $\mathcal{C}$ of positive equilibrium pairs $\left(r_{0}, x\right)$ outside the neighborhood of the bifurcation point $\left(r_{0}, x\right)=(1,0)$. The equation

$$
1=r_{0} \exp \left(-c x+\alpha \frac{s x}{1+s x}\right)
$$


satisfied by positive equilibria $x>0$, when re-written as

$$
r_{0}=\exp \left(c x-\alpha \frac{s x}{1+s x}\right)
$$

describes the continuum $\mathcal{C}$ of positive equilibrium pairs $\left(r_{0}, x\right)$. The graph of $r_{0}$ as a function of $x$ contains the point $r_{0}=1$ at $x=0$, decreases as $x$ increases to a unique critical point $x_{c r}>0$ at which $r_{0}$ attains a global minimum $r_{c r}>0$, and increases without bound for $x>x_{c r}$. See Figure 1. From the parabola-like shape of this graph, we see that the inverse function, treating $x$ as a function of $r_{0}$, has two branches: an upper branch of positive equilibria $x_{2}\left(r_{0}\right)$ for $r_{0} \geq r_{c r}$ and a lower branch of positive equilibria $x_{1}\left(r_{0}\right)<x_{2}\left(r_{0}\right)$ for $r^{c r} \leq r_{0}<1$ which satisfies $x_{1}(1)=0$. The value $r_{0}=r_{c r}$ is a saddle-node (blue-sky) bifurcation (or tipping) point at which the lower branch $x_{1}\left(r_{0}\right)$ and and upper branch $x_{2}\left(r_{0}\right)$ coalesce. The following facts follow from general results in [11]: for $r_{0}<r_{0}^{c r}$ the extinction equilibrium is globally asymptotically stable; the equilibria $x_{1}\left(r_{0}\right)$ are unstable and the equilibria $x_{2}\left(r_{0}\right)$ are (locally asymptotically) stable for $r_{0} \gtrsim r_{c r}$. The upper branch $x_{2}\left(r_{0}\right)$ might not remain stable for all $r_{0}>r_{c r}$, however, but might undergo a period doubling cascade to chaos. If a destabilization of $x_{2}\left(r_{0}\right)$ occurs at a point $r_{0} \geq 1$, then on the interval $r_{c r}<r_{0}<1$ there are two stable equilibria, the extinction equilibrium and the positive equilibrium $x_{2}\left(r_{0}\right)$. This scenario is called a strong Allee effect. It asserts that survival is possible for some $r_{0}<1$ provided a population's initial condition lies outside the basin of attractor of the extinction equilibrium (the Allee basin). If, on the other hand, $x_{2}\left(r_{0}\right)$ loses stability at a point in the interval $r_{c r}<r_{0}<1$, then there still occurs a strong Allee effect but one with a non-equilibrium survival attractor (e.g. a periodic cycle or a more complicated attractor).

Sample forward and backward bifurcation diagrams are shown in Figure 2. That secondary period-doubling bifurcations cascade to complex (presumably chaotic) dynamics in both cases is not unexpected, given that (23) is based on the Ricker nonlinearity. The backward bifurcation in Figure $2 \mathrm{~B}$ is an example illustrating a saddle-node bifurcation (at $r_{0} \approx 0.4$ ) that results a multi-stable equilibrium (strong Allee) scenario, as shown in Figure 1. In this example the positive equilibria destabilize (into a period doubling route to chaos) just outside the Allee interval $0.4<r_{0}<1$. In other examples, using different parameter values, this destabilization can occur at a value of $r_{0}<1$ so that the multi-attractor scenario of the strong Allee effect involves a stable cycle or even more complicated attractor. For examples and further results concerning the relationship between backward bifurcations and strong Allee effects, see [10]. The complex dynamics that can arise in this model, particularly when positive non-equilibrium attractors are present for $r_{0}<1$ are studied in [25], although not from this bifurcation point-of-view.

\section{INSERT FIGURES 1 and 2 NEAR HERE}

We now consider an evolutionary version of equation (23) to which we can apply the results of Sections 4 and 5. For our application we consider the case when the inherent (density and predation free) per capita birth rate $b$ and the predation protection factor $s$ are subject to evolutionary adaptation. We think of these per capita quantities as characteristics of an individual and that they are determined by a suite of phenotypic traits $\hat{v}$ of the individual. Thus, $b=b(\hat{v})$ and $s=s(\hat{v})$. We assume that there is a trait vector that maximizes $b$ and one that maximizes $s$, but these optimizing trait vectors are not the same. The idea is that there are trade-offs in the allocation of energy, behavioral activities, and resources towards reproduction and towards the avoidance of 
predators. For example, traits that promote physiological and behavioral characteristics promote successful herding or flocking or schooling in order to avoid predation are not necessarily traits that make for optimal reproduction.

Since we have set no units or scales for the traits, we assume without loss in generality that $b$ is maximal at $\hat{v}=\hat{0}$ and $s$ is maximal at $\hat{v}=(1,1, \cdots, 1)^{T}$. Specifically, we assume (as is often done in evolutionary models [28]) that these coefficients have a multi-variate Gaussian-type distribution about these maximal points:

$$
b(\hat{v})=\beta \exp \left(-\sum_{i=1}^{n} \frac{v_{i}^{2}}{2 b_{i}}\right), \quad s(\hat{v})=s_{0} \exp \left(-\sum_{i=1}^{n} \frac{\left(v_{i}-1\right)^{2}}{2 s_{i}}\right)
$$

where $b_{i}$ and $s_{i}$ are positive real numbers (variances), $\beta>0$ is the maximal possible value of $b(\hat{v})$, and $s_{0} \geq 0$ is the maximal possible value of $s(\hat{v})$. The resulting $1 \times 1$ projection matrix $P(x, \hat{u}, \hat{v})$ for $(23)$ is independent of $\hat{u}$ and its single entry $p_{11}(x, \hat{v})$ equals the dominant eigenvalue, i.e. $p_{11}(x, \hat{v})=r(x, \hat{v})$ where

$$
r(x, \hat{v})=\beta e^{-\alpha} \exp \left(-\sum_{i=1}^{n} \frac{v_{i}^{2}}{2 b_{i}}\right) \exp \left(-c x+\alpha \frac{s(\hat{v}) x}{1+s(\hat{v}) x}\right) .
$$

The Darwinian equations (6) are

$$
\begin{aligned}
x(t+1) & =r(x(t), \hat{u}(t)) x(t) \\
\hat{u}(t+1) & =\hat{u}(t)+M \nabla_{\hat{v}} \ln r(x(t), \hat{u}(t))
\end{aligned}
$$

with

$$
\nabla_{\hat{v}} \ln r(x, \hat{v})=-\left(\begin{array}{c}
\frac{v_{1}}{b_{1}} \\
\vdots \\
\frac{v_{n}}{b_{n}}
\end{array}\right)-\alpha \frac{s(\hat{v}) x}{(1+s(\hat{v}) x)^{2}}\left(\begin{array}{c}
\frac{v_{1}-1}{s_{1}} \\
\vdots \\
\frac{v_{n}-1}{s_{n}}
\end{array}\right) .
$$

Since

$$
\left.\nabla_{\hat{v}} \ln r(0, \hat{v})\right|_{\hat{v}=\hat{u}}=-\left(\begin{array}{c}
\frac{u_{1}}{b_{1}} \\
\vdots \\
\frac{u_{n}}{b_{n}}
\end{array}\right)
$$

we see that the only critical trait is

$$
\hat{u}^{*}=\hat{0} .
$$

and hence the only extinction equilibrium is $(x, \hat{u})=(0, \hat{0})$. Our bifurcation parameter $r_{0}^{*}=r(0, \hat{0})$ reduces to

$$
r_{0}^{*}=\beta e^{-\alpha} .
$$

Under the added assumption that the traits are not correlated, so that the variance-covariance matrix $M=\operatorname{diag}\left(\sigma_{i}^{2}\right)$ is a diagonal matrix, the model equations for our evolutionary version of (23) are

$$
\begin{aligned}
& x(t+1)=r_{0}^{*} \exp \left(-\sum_{i=1}^{n} \frac{u_{i}^{2}(t)}{2 b_{i}}\right) x(t) \exp \left(-c x(t)+\alpha \frac{s(\hat{u}(t)) x(t)}{1+s(\hat{u}(t)) x(t)}\right) \\
& u_{i}(t+1)=u_{i}(t)-\sigma_{i}^{2}\left(\frac{u_{i}(t)}{b_{i}}+\alpha \frac{u_{i}(t)-1}{s_{i}} \frac{s(\hat{u}(t)) x(t)}{(1+s(\hat{u}(t)) x(t))^{2}}\right)
\end{aligned}
$$


for $i=1, \cdots, n$. Our goal is to apply Corollary 4 to these difference equations, toward which end we must consider $\mathrm{H} 2$ and $\mathrm{H} 5$.

$\mathrm{H} 2$ holds with $\tilde{p}_{11}(\hat{v})=r_{0}(\hat{v})$ and $\bar{p}(x, \hat{u}, \hat{v})=\bar{r}(x, \hat{v})$, since $\bar{r}(0, \hat{v}) \equiv 1$. A calculation shows that the Hessian $H\left(0, \hat{u}^{*}, \hat{u}^{*}\right)=\left[\partial_{v_{i} v_{j}}^{0} \ln \bar{r}\right]$ is the diagonal matrix

$$
H\left(0, \hat{u}^{*}, \hat{u}^{*}\right)=\operatorname{diag}\left[-\frac{1}{b_{i}}\right]
$$

Thus, H5 holds (see Remark 4). By Theorem 3 the bifurcating continuum of positive equilibrium pairs $\left(r_{0}^{*},(x, \hat{u})\right)$, near the bifurcation point $(1,(0, \hat{0}))$, has the parametric representation

$$
\begin{aligned}
r_{0}^{*}(\varepsilon) & =1+\kappa^{*} \varepsilon+O\left(\varepsilon^{2}\right) \\
x(\varepsilon) & =\varepsilon+O\left(\varepsilon^{2}\right) \\
\hat{u}(\varepsilon) & =\hat{u}_{1} \varepsilon+O\left(\varepsilon^{2}\right)
\end{aligned}
$$

for $\varepsilon \gtrsim 0$ where, by the formulas (18) and (19),

$$
\begin{gathered}
\kappa^{*}=c-\alpha s_{0} \exp \left(-\sum_{i=1}^{n} \frac{1}{2 s_{i}}\right) \\
\hat{u}_{1}=\alpha s_{0} \exp \left(-\sum_{i=1}^{n} \frac{1}{2 s_{i}}\right)\left(\begin{array}{c}
\frac{b_{1}}{s_{1}} \\
\vdots \\
\frac{b_{n}}{s_{n}}
\end{array}\right) \in \mathbb{R}_{+}^{n} .
\end{gathered}
$$

By Corollary $4(\mathrm{a}, \mathrm{b})$, the direction of bifurcation determines the stability of the bifurcating positive equilibria provided

$$
\sigma_{i}^{2}<2 b_{i} \text { for all } i=1, \cdots, n
$$

that is to say, provided the speed of evolution is not too fast. Under this assumption, we have the following conclusions concerning the bifurcation at $r_{0}^{*}=1$ for the Darwinian model (24).

1. (Forward bifurcations) The bifurcation of the continuum $\mathcal{C}$ of positive equilibria for (24b) is forward and consequently stable if

$$
\alpha s_{0} \exp \left(-\sum_{i=1}^{n} \frac{1}{2 s_{i}}\right)<c .
$$

This occurs if the negative density effects, as described by the Ricker coefficient $c$, are large enough to dominate the positive effects from the Allee effect attributed to density protection from predation, as encapsulated by the quantity on the left side of the inequality (27). Thus, mechanisms that promote a forward bifurcation are: a low predation intensity $\alpha$, a low maximum possible predation protection coefficient $s_{0}$, and small variances $s_{i}$ (i.e. the largest predator protection coefficients $s(\hat{v})$ are attained only for trait vectors narrowly distributed around the maximal trait vector $\hat{v}=$ $\left.(1, \cdots, 1)^{T}\right)$.

Note that the entries in $\hat{u}_{1}$ in $(25 \mathrm{c})$ are positive if $\alpha s_{0}>0$, i.e. if both predation and predation protection are present. In this case, we see that near the bifurcation point, the trait components $u_{i}(\varepsilon)$ of the bifurcating positive equilibria are positive. As a result, for $r_{0}^{*} \gtrsim 1$ the stable, positive 
equilibria have trait components that do not maximize the inherent birth rate. Indeed, an even stronger conclusion follows directly from the trait equilibrium equations (24b):

$$
\frac{u_{i}}{b_{i}}+\alpha \frac{u_{i}-1}{s_{i}} \frac{s(\hat{u}) x}{(1+s(\hat{u}) x)^{2}}=0 \text { for all } i=1, \ldots, n .
$$

This shows, when $\alpha>0$ and $s_{0}>0$, that for any positive equilibrium $(x, \hat{u})$ of $(24)$, the equilibrium trait components $u_{i}$ cannot equal 0 or 1 . For those equilibrium pairs from the continuum $\mathcal{C}$ the trait components form a continuum of equilibrium trait vectors $\hat{u}$ which must, therefore, have components that lie entirely in the interval $0<u_{i}<1$ (whether the equilibria are stable or not). It follows that for those positive equilibria from $\mathcal{C}$ which are in fact stable (such as those for $r_{0}^{*} \gtrsim 1$ ), we can say that evolution selects a vector of traits that neither maximizes the inherent birth rate $b(\hat{u})$ (which occurs at $\hat{u}=\hat{0}$ ) nor the predator protection coefficient $s(\hat{u})$ (which occurs at $\hat{u}=(1,1, \cdots, 1)^{T}$ ). One might say, then, that evolution trades-off a smaller inherent birth rate in favor of some predator protection.

When predation and/or predation protection is absent $\left(\alpha=0\right.$ and/or $\left.s_{0}=0\right)$ in the model, then clearly inequality (27) holds and the bifurcation at $r_{0}^{*}=1$ is forward and stable. In this case, the equilibrium equation (28) for the traits $u_{i}$ implies $u_{i}=0$ for any positive equilibrium pair and, not surprisingly, evolution selects to maximize the inherent bifurcation rate $b(\hat{u})$.

(2) (Backward bifurcations). The bifurcation of the continuum $\mathcal{C}$ of positive equilibria for (24b) is backward and consequently unstable if

$$
\alpha s_{0} \exp \left(-\sum_{i=1}^{n} \frac{1}{2 s_{i}}\right)>c .
$$

This occurs only if predation is present $\alpha>0$ and density protection from predation is also present $s_{0}>0$. Inequality (29) holds if predation intensity $\alpha$ and/or predator protection $s_{0}$ are large (relative to the negative density effects $c$ ). Also promoting a backward bifurcation are large variances $s_{i}$, that is to say, when a high level of predator protection $s(\hat{v})$ is attained for a wide distribution of trait vectors $\hat{v}$.

Our general results in Section 5 concern equilibrium properties in a neighborhood of the bifurcation point and do not imply anything about the dynamics outside such a neighborhood. As in the non-evolutionary model (23), we expect it to be true that the positive equilibria on the continuum $\mathcal{C}$ for the evolutionary model (24) do not necessarily retain the stability properties that they possess near the bifurcation point. In particular, in the case of a forward/stable bifurcation we would expect that, at least for some model parameter values, the stable positive equilibria will destabilize with increasing $r_{0}^{*}$ and even give rise to a sequence of bifurcations that result complicated, chaotic dynamics. In the case of a backward/unstable bifurcation, in addition to this phenomenon, we would also anticipate the potential for strong Allee effects on an interval of $r_{0}^{*}$ values less than 1. We will not study these questions about the dynamics of (24) in this paper where our theory is focussed on the local bifurcation at $r_{0}^{*}=1$.

However, we can provide a few selected numerical simulations that, in addition to illustrating the local bifurcation predicted by our theorems, also illustrate the kinds of secondary bifurcations and strong Allee effects since in the non-evolutionary case (cf. Figure 2). Figure 3 shows two sample bifurcation diagrams for the evolutionary model (24) with two traits, i.e. $n=2$. The plots in Figure $3 \mathrm{~A}$ are from parameter values for which $\kappa^{*}>0$ and, hence, a forward, stable bifurcation occurs at 
$r_{0}^{*}=1$. As with the non-evolutionary version of the model in Figure 1, further increases in $r_{0}^{*}$ result in the familiar period doubling route to chaotic dynamics. In Figure 3B the same parameter values are used except that the predator protection coefficient $s_{0}$ is increased to the extent that $\kappa^{*}<0$ and, as a result, a backward, unstable bifurcation occurs. The result is a bifurcation diagram that shows a saddle-node bifurcation (at $r_{0}^{*} \approx 0.4$ ) creating an interval of strong Allee effects with both a stable extinction equilibrium $(x, \hat{u})=(0, \hat{0})$ and a stable positive equilibrium. In this example, one sees from Figure 3B that the positive equilibrium loses stability through a period doubling at a value of $r_{0}^{*}$ less than 1 . This results in an interval of $r_{0}^{*}$ values less than 1 for which there is a strong Allee effect that involves a stable positive 2-cycle instead of a positive equilibrium. (The oscillations in the traits $u_{i}$ are small amplitude in Figure 3B.)

\section{INSERT FIGURE 3 NEAR HERE}

\section{Concluding Remarks}

A fundamental property of population dynamic models, when the extinction state destabilizes due to a change in a model parameter, is the occurrence of a bifurcation which results in the presence of positive equilibria. Typically the stability of these bifurcating equilibria depend on the direction of bifurcation (Theorem 1). In this paper we investigate this basic bifurcation phenomenon for an evolutionary version of a general matrix model for the dynamics of a structured population. The model assumes that the entries of the model's projection matrix (i.e. the per capita birth, survival and class transition rates) depend on a vector of phenotypic traits, each of which is subject to Darwinian evolution, and tracks the dynamics of the population and the vector of mean traits [28]. We define the notion of a critical trait vector, which is associated with the existence of an extinction equilibrium in the model, and obtain conditions under which an extinction equilibrium destabilizes (Theorem 2) and conditions under which a continuum of positive equilibria bifurcates from the extinction equilibrium, as the inherent population growth rate (at the critical trait) increases through 1 (Theorem 3). We further obtain conditions under which stability of the bifurcating equilibria is determined by the direction of bifurcation and conditions under which it is not (Theorem 3).

It is shown in [23] that the bifurcating continuum $\mathcal{C}^{*}$ of positive equilibria in Theorem 3 has a global extent in $\mathbb{R}_{+} \times\left(\mathbb{R}_{+}^{m} \times V\right)$ in that it connects to the boundary of this cone $(\infty$ is included in the boundary). In general, however, the stability/instability results in Theorem 3 hold only in a neighborhood of the bifurcation point. This is illustrated in the example studied in Section 6 where secondary bifurcations occur outside the neighborhood of the bifurcation point. Whether or not such bifurcations occur are model dependent (which is true in non-evolutionary matrix models as well).

In non-evolutionary matrix models, backward bifurcations are often associated with strong Allee effects, i.e. multiple attractors for values of $r_{0}^{*}<1$ one of which is extinction and the other which is a survival attractor [10]. While conditions sufficient for the occurrence of a backward bifurcation are given in Section 5, its relation to strong Allee effects is not investigated in this paper. A backward induced strong Allee effect is shown to occur, by simulations, in the example studied in Section 6 . 


\section{Acknowledgements}

J. M. Cushing and A. Veprauskas were supported by the U.S. National Science Foundation grant DMS 0917435.

A. A. Pinto thanks the support of LIAAD - INESC TEC through program PEst, the Faculty of Sciences of University of Porto and Portuguese Foundation for Science and Technology (FCT - Fundação para a Ciência e a Tecnologia) through the Project "Dynamics, Optimization and Modelling, with with reference PTDC/MAT-NAN/6890/2014. This work is financed by the ERDF - European Regional Development Fund through the Operational Programme for Competitiveness and Internationalisation - COMPETE 2020 Programme, and by National Funds through the FCT - Fundação para a Ciência e a Tecnologia (Portuguese Foundation for Science and Technology) within project «POCI-01-0145-FEDER-006961.

F. Martins thanks the financial support of Portuguese Foundation for Science and Technology (FCT - Fundação para a Ciência e a Tecnologia) through a PhD. scholarship of the programme MAP-PDMA. (Reference: PD/BD/105726/2014).

The authors are grateful for the comments of two anonymous reviewers and the handling editor, which were exceptionally helpful in improving the paper.

\section{Appendix}

Lemma 1 Assume H2 and H5 hold. Then $\hat{w}_{L}^{T}\left[\nabla_{\hat{v}}^{0} q_{i j}^{T} \hat{u}_{1}\right] \hat{w}_{R}=0$.

Proof. Consider the equality

$$
P(\hat{0}, \hat{u}, \hat{u}) \hat{w}_{R}(\hat{0}, \hat{u})=r(\hat{0}, \hat{u}, \hat{u}) \hat{w}_{R}(\hat{0}, \hat{u}) .
$$

which holds by the definition of $r(\hat{0}, \hat{u}, \hat{u})$ as an eigenvalue with a positive right eigenvector $\hat{w}_{R}(\hat{0}, \hat{u})$. Let $\hat{p}_{i}=\hat{p}_{i}(\hat{0}, \hat{u}, \hat{u})$ denote the $i$-th column of $P=P(\hat{0}, \hat{u}, \hat{u})$. We want to take the Jacobian of both sides of equation (30) with respect to $\hat{u}$. To do this we let $J_{\hat{y}}[\hat{\omega}(\hat{y})]$ denote the Jacobian of a vector valued function $\hat{\omega}(\hat{y})$ of a vector $\hat{y}$.

The right side of $(30)$ is a vector valued function of the form $\tau(\hat{y}) \hat{\omega}(\hat{y})$ for a scalar valued function $\tau(\hat{y})$. Applying the general formula

$$
J_{\hat{y}}[\tau(\hat{y}) \hat{\omega}(\hat{y})]=\hat{\omega}(\hat{y}) \nabla_{\hat{y}} \tau(\hat{y})^{T}+\tau(\hat{y}) J_{\hat{y}}[\hat{\omega}(\hat{y})]
$$

and recalling (8) in Remark 2, we find that the Jacobian of the right side of (30) with respect to $\hat{u}$ is

$$
\hat{w}_{R}(\hat{0}, \hat{u})\left(\nabla_{\hat{u}} r^{T}+\nabla_{\hat{v}} r^{T}\right)+r J_{\hat{u}}\left[\hat{w}_{R}(\hat{0}, \hat{u})\right]=\hat{w}_{R}(\hat{0}, \hat{u}) \nabla_{\hat{v}} r^{T}+r J_{\hat{u}}\left[\hat{w}_{R}(\hat{0}, \hat{u})\right] .
$$

To calculate the Jacobian of the left-hand side of (30), we write

$$
P \hat{w}_{R}(\hat{0}, \hat{u})=\sum_{i=1}^{m} w_{i}^{R}(\hat{0}, \hat{u}) \hat{p}_{i}
$$

where $w_{i}^{R}(\hat{0}, \hat{u})$ are the components of the vector $\hat{w}_{R}(\hat{0}, \hat{u})$ and apply the product rule (31) to each term. Noting (7) in Remark 2 we get

$$
P J_{\hat{u}}\left[\hat{w}_{R}(\hat{0}, \hat{u})\right]+\sum_{i=1}^{m} w_{i}^{R}(\hat{0}, \hat{u}) J_{\hat{v}}\left[\hat{p}_{i}\right] .
$$


Equating the Jacobians of the left and right sides of (30) we have

$$
P J_{\hat{u}}\left[\hat{w}_{R}(\hat{0}, \hat{u})\right]+\sum_{i=1}^{m} w_{i}^{R}(\hat{0}, \hat{u}) J_{\hat{v}}\left[\hat{p}_{i}\right]=\hat{w}_{R}(\hat{0}, \hat{u}) \nabla_{\hat{v}} r^{T}+r J_{\hat{u}}\left[\hat{w}_{R}(\hat{0}, \hat{u})\right]
$$

or

$$
\left(P-r I_{m}\right) J_{\hat{u}}\left[\hat{w}_{R}(\hat{0}, \hat{u})\right]=\hat{w}_{R}(\hat{0}, \hat{u}) \nabla_{\hat{v}} r^{T}-\sum_{i=1}^{m} w_{i}^{R}(\hat{0}, \hat{u}) J_{\hat{v}}\left[\hat{p}_{i}\right]
$$

which in turn can be rewritten as the $m$ equations

$$
\left(P-r I_{m}\right) \partial_{u_{i}}\left(\hat{w}_{R}(\hat{0}, \hat{u})\right)=\left(\partial_{v_{i}} r I_{m}-\partial_{v_{i}} P\right) \hat{w}_{R}(\hat{0}, \hat{u}) \text { for } 1 \leq i \leq n .
$$

The matrix $P-r I_{m}$ is singular and by the Fredholm alternative, the solubility of these equations imply the $m$ orthogonality conditions

$$
\hat{w}_{L}^{T}(\hat{0}, \hat{u})\left(\partial_{v_{i}} r I_{m}-\partial_{v_{i}} P\right) \hat{w}_{R}(\hat{0}, \hat{u})=0
$$

are satisfied. Solving for $\partial_{v_{i}} r$ and recalling that the eigenvectors are normalized so that $\hat{w}^{L}(\hat{0}, \hat{u})^{T} \hat{w}_{R}(\hat{0}, \hat{u})=$ 1 , we find

$$
\partial_{v_{i}} r=\hat{w}_{L}^{T}(\hat{0}, \hat{u}) \partial_{v_{i}} P \hat{w}_{R}(\hat{0}, \hat{u}) \text { for } 1 \leq i \leq n .
$$

Since $\partial_{v_{i}}^{0} r=0$ by definition of a critical trait vector $\hat{u}^{*}$, when setting $\hat{u}=\hat{u}^{*}$ and $r_{0}^{*}=1$ in these expressions we get

$$
\hat{w}_{L}^{T} \partial_{v_{k}}^{0} Q \hat{w}_{R}=0 \text { for } 1 \leq k \leq n .
$$

Let $u_{1, k}$ denote the scalar components of the vector $\hat{u}_{1}$. Then

$$
\nabla_{\hat{v}}^{0} q_{i j}^{T} \hat{u}_{1}=\sum_{k=1}^{n} u_{1, k} \partial_{v_{k}}^{0} q_{i j}
$$

and

$$
\left[\nabla_{\hat{v}}^{0} q_{i j}^{T} \hat{u}_{1}\right]=\sum_{k=1}^{n} u_{1, k}\left[\partial_{v_{k}}^{0} q_{i j}\right]=\sum_{k=1}^{n} u_{1, k} \partial_{v_{k}}^{0} Q
$$

From

$$
\hat{w}_{L}^{T}\left[\nabla_{\hat{v}}^{0} q_{i j} \hat{u}_{1}\right] \hat{w}_{R}=\sum_{k} u_{1, k}\left(\hat{w}^{L} \partial_{v_{k}}^{0} Q \hat{w}_{R}\right)
$$

and (33) it follows that $\hat{w}_{L}^{T}\left[\nabla_{\hat{v}}^{0} q_{i j} \hat{u}_{1}\right] \hat{w}_{R}=0$. 


\section{References}

[1] P. A. Abrams, Modelling the adaptive dynamics of traits involved in inter- and intraspecific interactions: An assessment of three methods, Ecology Letters, 4 (2001), pp. 166-175.

[2] P. A. Abrams, 'Adaptive Dynamics' vs. 'adaptive dynamics', Journal of Evolutionary Biology, 18 (2005), pp. 1162-1165.

[3] A. Bernman and R. J. Plemmons, Nonnegative Matrices in the Mathematical Sciences, Classics in Applied Mathematics. SIAM, Philadelphia, 1994.

[4] H. Caswell, Matrix Population Models: Construction, Analysis and Interpretation, Second Edition, Sinauer Associates, Inc. Publishers, Sunderland, Massachusetts, 2001.

[5] F. Courchamp, L. Berec and J. Gascoigne, Allee Effects in Ecology and Conservation, Oxford University Press, Oxford, Great Britain, 2008.

[6] J. M. Cushing, A bifurcation theorem for Darwinian matrix models, Nonlinear Studies, 17 (2010), pp. 1-13.

[7] J. M. Cushing, An Introduction to Structured Population Dynamics, CBMS-NSF Regional Conference Series in Applied Mathematics, Vol. 71, SIAM, Philadelphia, 1998.

[8] J. M. Cushing, Matrix Models and Population Dynamics, a chapter in Mathematical Biology (Mark Lewis, A. J. Chaplain, James P. Keener, Philip K. Maini eds.), IAS/Park City Mathematics Series Vol. 14, American Mathematical Society, Providence, I, 2009, pp. 47-150.

[9] J. M. Cushing, On the relationship between $r$ and $R_{0}$ and its role in the bifurcation of equilibria of Darwinian matrix models, Journal of Biological Dynamics, 5 (2011), pp. 277-297.

[10] J. M. Cushing, Backward bifurcations and strong Allee effects in matrix models for the dynamics of structured populations, Journal of Biological Dynamics, 8 (2014), pp. 57-73.

[11] J. M. Cushing, One dimensional maps as population and evolutionary dynamic models, Applied Analysis in Biological and Physical Sciences, Springer Proceedings in Mathematics \& Statistics, Volume 186, (J. M. Cushing, M. Saleem, H. M. Srivastava, M. A. Khan, M. Merajuddin, eds.), Springer, India, 2016.

[12] B. Dennis, Allee effects: Population growth, critical density, and the chance of extinction, Natural Resource Modeling 3 (1989), 481-538.

[13] F. Dercole and S. Rinaldi, Analysis of Evolutionary Processes: The Adaptive Dynamics Approach and Its Applications, Princeton University Press, New Jersey, 2008

[14] S. N. Elaydi, An Introduction to Difference Equations, Springer-Verlag, New York, 1996.

[15] H. Keilhöfer, Bifurcation Theory: An Introduction with Applications to PDEs, Applied Mathematical Sciences 156, Springer, New York, 2004.

[16] R. Lande, Natural selection and random genetic drift in phenotypic evolution, Evolution, 30 (1976), pp. 314-334. 
[17] R. Lande, A quantitative genetic theory of life history evolution, Ecology, 63 (1982), pp. 607615.

[18] L. P. Lefkovitch, The study of population growth in organisms grouped by stage, Biometrics, 21 (1965), 1-18

[19] P. H. Leslie, On the use of matrices in certain population mathematics, Biometrika, 33 (1945), pp. 183-212.

[20] P. H. Leslie, Some further notes on the use of matrices in population mathematics, Biometrika, 35 (1948), 213-245.

[21] E. G. Lewis, On the generation and growth of a population, Sankhya, 6 (1942), 93-96.

[22] B. J. McGill and J. S. Brown, Evolutionary game theory and adaptive dynamics of continuous traits, Annual Review of Ecology, Evolution, and Systematics, 38 (2007), pp. 403-435.

[23] E. P. Meissen, K. R. Salau, J. M. Cushing, A global bifurcation theorem for Darwinian matrix models, to appear in the Journal of Difference Equations and Applications

[24] D. A. Roff, The Evolution of Life Histories: Theory and Analysis, Chapman and Hall, New York, 1992.

[25] S. Schreiber, Allee effects, extinctions, and chaotic transients in simplie population models, Theoretical Population Biology 64 (2003), 201-209.

[26] M. B. Usher, A matrix approach to the management of renewable resources, with special reference to selection forests, Journal of Applied Ecology 3 (1966), 355-367.

[27] A. Veprauskas and J. M. Cushing, Evolutionary dynamics of a multi-trait semelparous model, Discrete and Continuous Dynamical Systems Series B, 21, No. 2 (2016), 655-676.

[28] T. L. Vincent and J. S. Brown, Evolutionary Game theory, Natural selection, and Darwinian dynamics, Cambridge University Press, 2005. 


\section{Click here to view linked References}

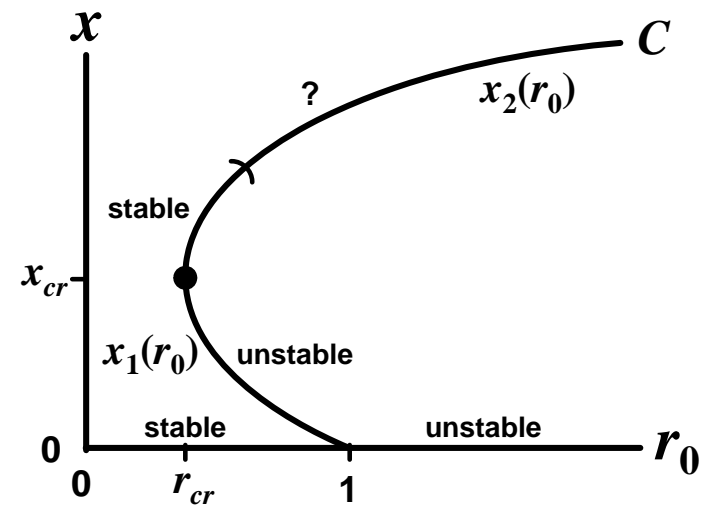

Figure 1. Shown is a generic plot of the bifurcating continuum $C$ for equation (23) when $\alpha s>c$ and, consequently, a backward (unstable) bifurcation occurs at the point $\left(r_{0}, x\right)=(1,0)$. The question mark indicates that although the positive equilibria on the upper branch $x_{2}\left(r_{0}\right)$ are (locally asymptotically) stable near the saddle-node bifurcation point $\left(r_{c r}, x_{c r}\right)$, they can, depending on model parameter values, destabilize further along the continuum $C$.
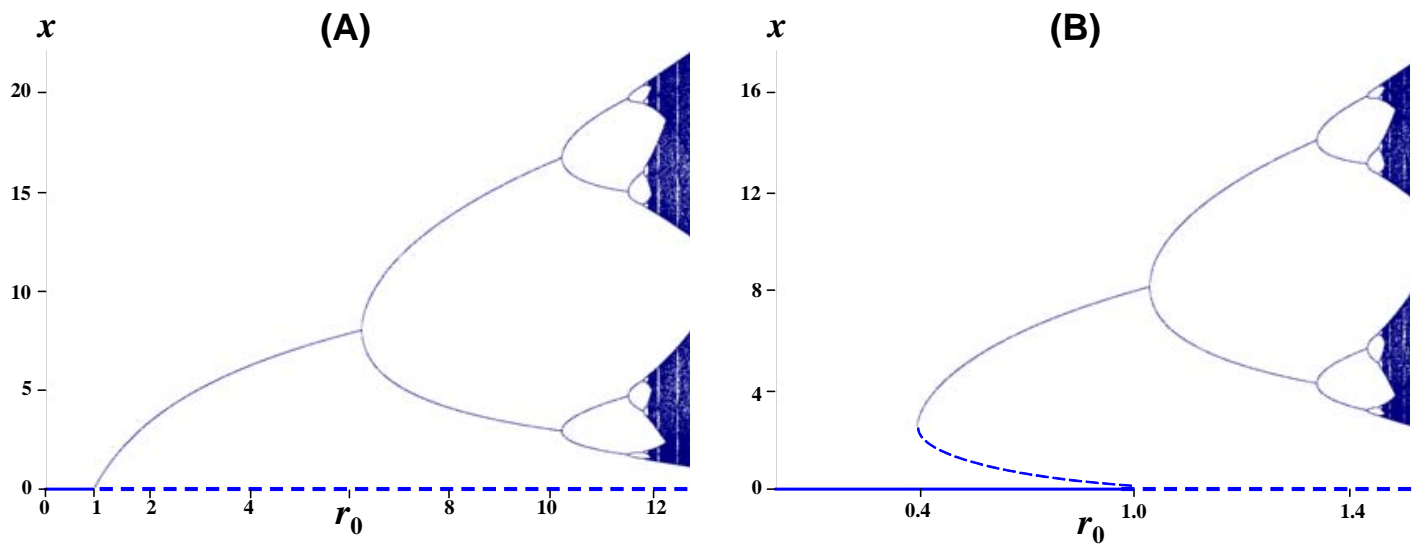

FiguRE 2. Sample bifurcation diagrams for equation (23) with $c=0.3$ and $\alpha=3$.

A. $s=0.05$ and $\kappa=c-\alpha s=0.15>0$ so that the bifurcation at $r_{0}=\beta e^{-3}=1$ is forward and stable.

B. $s=1$ and $\kappa=-2.7<0$ so that the bifurcation at $r_{0}=\beta e^{-3}=1$ is backward and unstable (dashed line). 

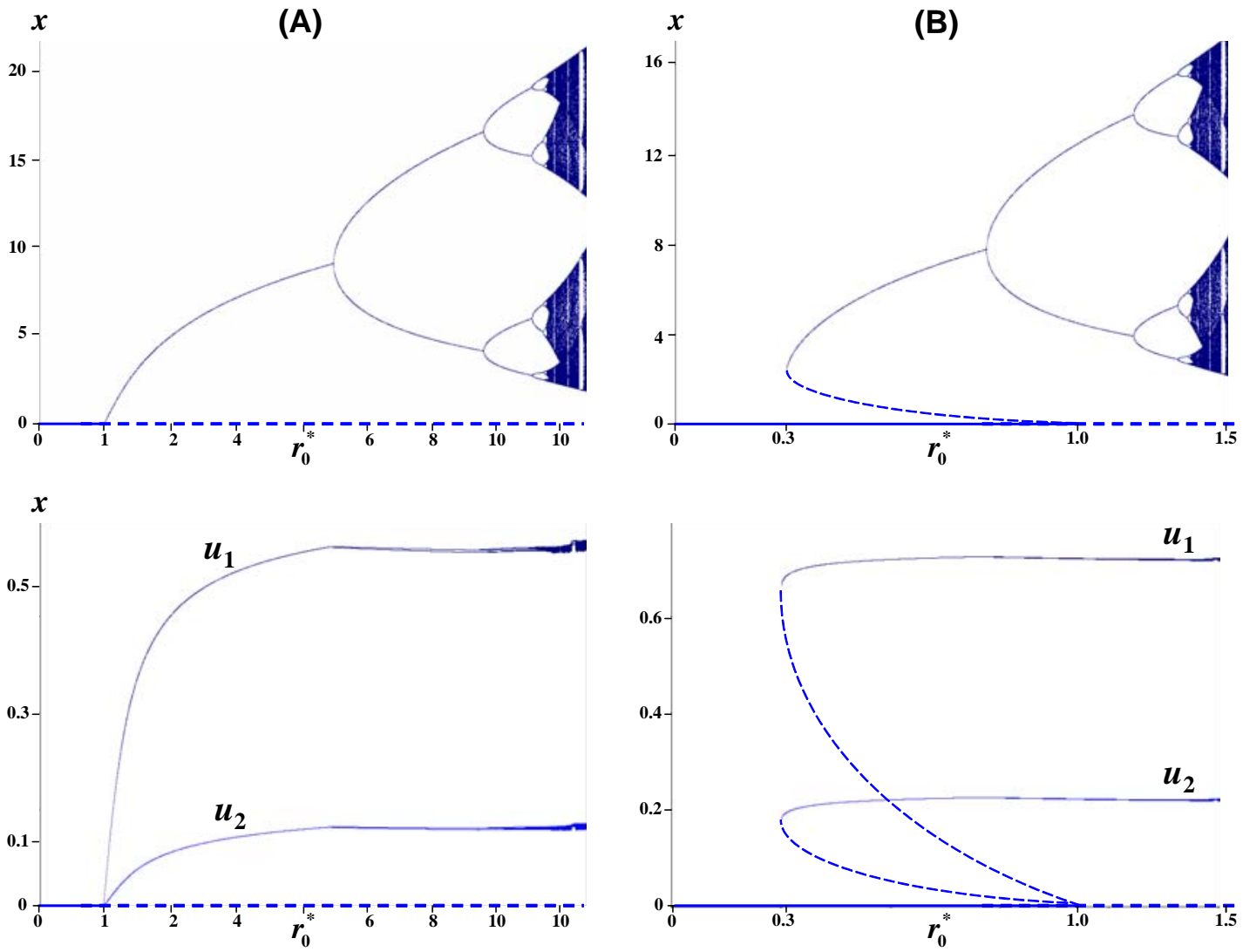

FiguRE 3. Sample bifurcation diagrams for equations (24) with $c=0.3, \alpha=3, b_{1}=$ $3, b_{2}=1, s_{1}=1$, and $s_{2}=3$.

A. $s_{0}=0.1$ and $\kappa^{*}=3\left(1-e^{-2 / 3}\right) / 10 \approx 0.146>0$ so that the bifurcation at $r_{0}^{*}=\beta e^{-3}=1$ is forward and stable.

B. $s=1$ and $\kappa^{*}=3\left(1-10 e^{-2 / 3}\right) / 10 \approx-1.2403<0$ so that the bifurcation at $r_{0}^{*}=\beta e^{-3}=1$ is backward and unstable (dashed lines). 
(A)
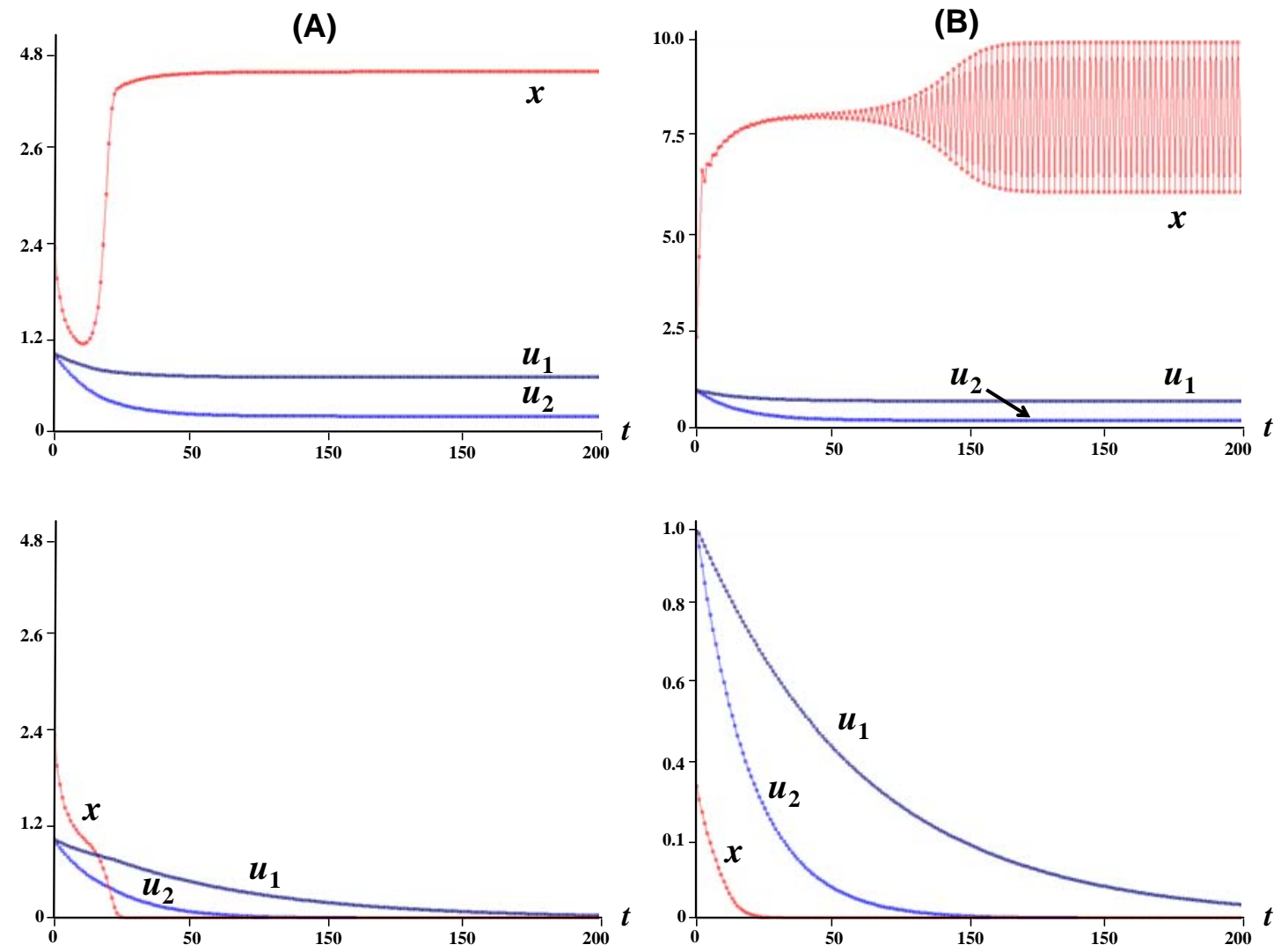

FIGURE 4. Shown are sample orbits for equations (24) with the same parameter values used in Figure 3B when a backward bifurcation creates an interval of $r_{0}^{*}<1$ for which there is a strong Allee effect.

A. For $r_{0}^{*}=\beta e^{-3}=0.4$ the bifurcation diagram Figure 3B shows a stable extinction equilibrium and a stable positive equilibrium. The upper graph in column A shows plots of the solution with initial conditions $x=2.35, u_{1}=u_{2}=1$ and that tends to the positive equilibrium. The lower graph shows plots of the solution with initial conditions $x=2.33, u_{1}=u_{2}=1$ and that tends to the extinction equilibrium $(x, \hat{u})=(0, \hat{0})$.

B. For $r_{0}^{*}=\beta e^{-3}=0.9$ the bifurcation diagram Figure 3B shows a stable extinction equilibrium and a stable 2-cycle. The upper graph in column B shows plots are of the solution with initial conditions $x=2.35, u_{1}=u_{2}=1$ and that tends to the positive 2-cycle. The lower graph in column B shows plots of the solution with initial conditions $x=0.35, u_{1}=u_{2}=1$ and that tends to the extinction equilibrium $(x, \hat{u})=(0, \hat{0})$. 